\title{
Theory of the Stationary Self-Consistent Universe
}

\section{Alexander Sh. Avshalumov,}

Head of Moscow Research Institute for Cybernetic Medicine (MRICM), Miklouho-Maclay str. 16/10, building 90, 117437, Moscow, Russia.

\section{ABSTRACT}

Since the creation of $G R$ and subsequent works in cosmology, the question of the curvature of space in the Universe ( $\omega>0, \omega<0$ and $\Omega=0$ ) is considered one of the most important and debated to this day. This is evident, because the curvature of space depends whether the Universe expands, contracts or is static. These discussions allowed the author to propose a paradoxical idea: simultaneous existence in the Universe of three interconnected spacetimes (positive, negative and zero curvature) and on this basis, to develop a theory in which each space-time plays its own role and develops in a strict accordance with its sign of curvature. The proposed work explores a hypothesis of new space-time symmetry in the Universe, according to which in the primary (maternal) generalized Absolute space-time ${ }^{A} S T$ of zero $(\Omega=0)$ curvature with Euclidean geometry $(\mathcal{E})$ when interacting with sphere with distance-radius and curvature, which are equal to imaginary parabolic unit $I$ ( $S_{s p h}=I$, $\Omega_{s p h}=I$ ) at the moment of the Big Bang ( $\left.B B\right)$, two mutually transparent noncommutative self-consistent space-times were synchronously born:

- Hot space-time ${ }^{L} S T$ of constant positive $(\omega>0)$ curvature with positive density of matter and energy, in which the elliptic Riemannian geometry $(\mathcal{R})$ is realized, and the tissue of space homogenously expands (relative to the tissue of Absolute space-time ${ }^{A} S T$ ) in time ${ }^{L} T$

- Cold space-time ${ }^{D} S T$ of constant negative $(\omega<0)$ curvature with negative density of matter and energy, in which the hyperbolic Lobachevsky-Bolyai geometry $(\mathcal{L B})$ is realized, and the tissue of space heterogeneously contracts (relative to the tissue of Absolute space-time ${ }^{A} S T$ ) in time ${ }^{D} T$

At any moment of synchronization time $\left(T_{\text {synch }}\right)$ the following equation is carried out $\left[{ }^{L} \omega\left(T_{\text {synch }}\right) \cdot{ }^{D} \omega\left(T_{\text {synch }}\right)=I\right]$, where $I=|\mathbf{1}|$.

This cosmological model allows to ultimately solve the problem of the observed baryon asymmetry and hidden mass in the Universe, since from the moment $B B$ all the born matter develops in the space-time we observe, of positive curvature ${ }^{L} S T$, and all the born antimatter develops in the not observed by us, space-time of negative curvature ${ }^{D} S T$.

This approach allows to recognize the three space-time nature of spin and develop its mechanism, which directly leads to a new three space-time self-consistent particle-soliton model of the atom and atomic nucleus, which allows to give a new interpretation of quantum mechanics. 


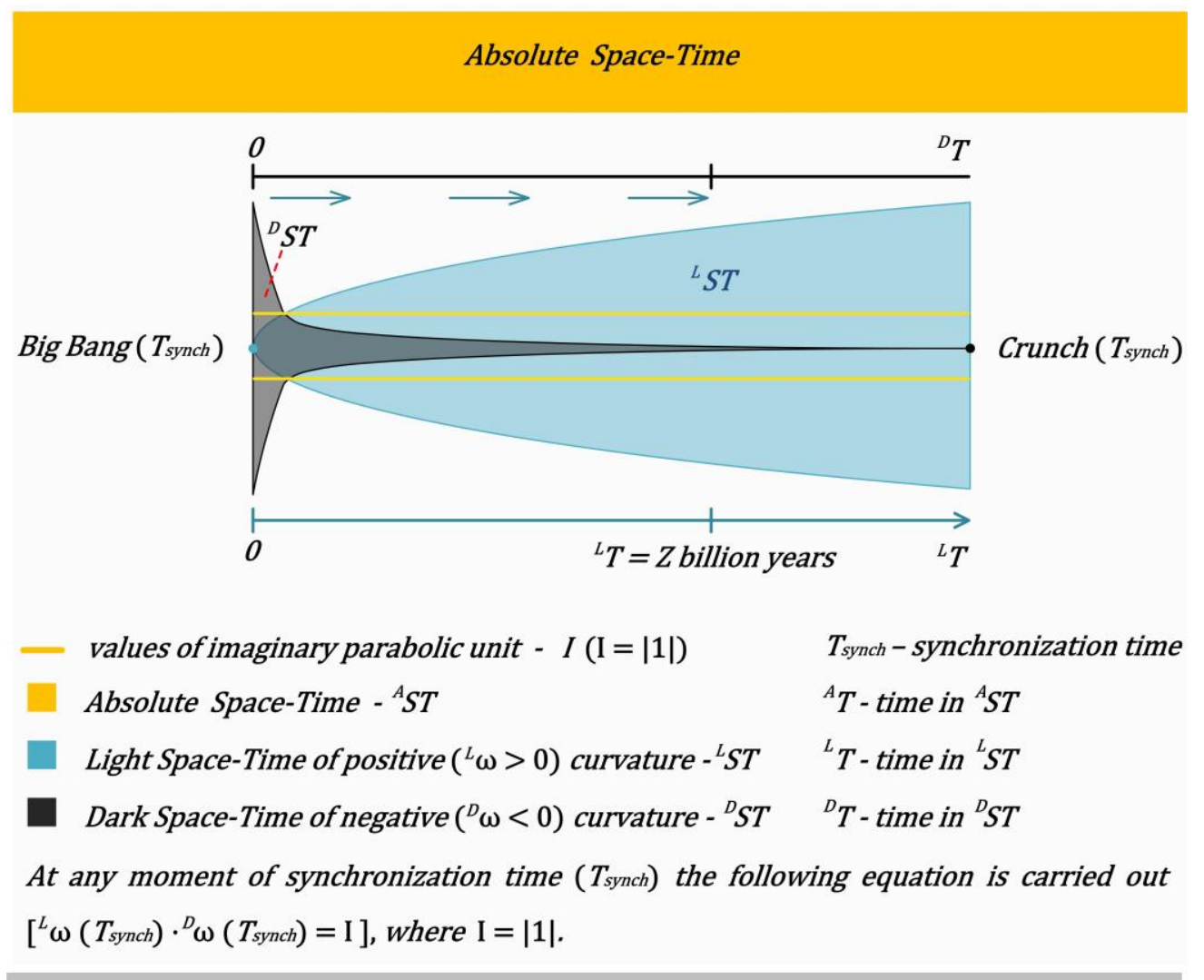

Graphical abstract: Three space-time model of the structure of the Universe.

The three space-time model of the structure of the Universe, proposed by the author, allows to solve many fundamental problems of modern cosmology and theoretical physics and creates the basis for building a unified physical theory (including one that unites GR and quantum physics). In the foreseeable future, this model also gives hope to develop a quantitative, logically complete model of the birth of $B B$ and the subsequent Crunch $\mathrm{Cr}$ of two mutually transparent non-commutative self-consistent space-times of constant positive and constant negative curvature. 


\section{Preamble}

«So maybe what we call imaginary time is really more basic, and what we call real is just an idea that we invent to help us describe what we think the Universe is like»

Stephen Hawking «A Brief History of Time», 1988.

«Quantum mechanics is certainly imposing. But an inner voice tells me that it is not yet the real thing. The theory says a lot but does not really bring us any closer to the secret of the "Old One." I, at any rate, am convinced that He is not playing at dice.».

Albert Einstein «Letter to Max Born», December 4, 1926

«...God is a mathematician of a very high order, and He used very advanced mathematics in constructing the Universe».

Paul Adrien Maurice Dirac «The Evolution of the Physicist's Picture of Nature», May issue Scientific American, 1963 


\section{Contents}

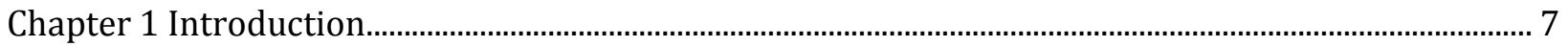

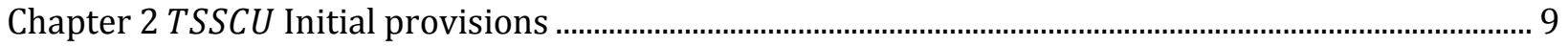

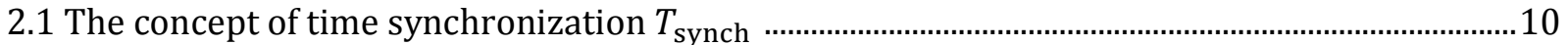

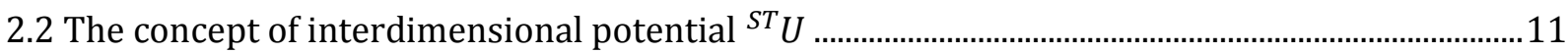

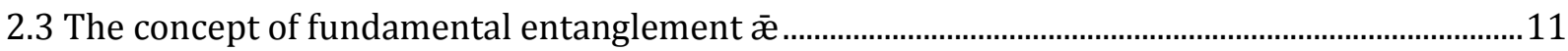

Chapter 3 Primary (maternal) Absolute space-time ${ }^{A} S T$ as the basis for the Big Bang $(B B)$ model and subsequent Crunch $(\mathrm{Cr})$..........................................................................................................................12

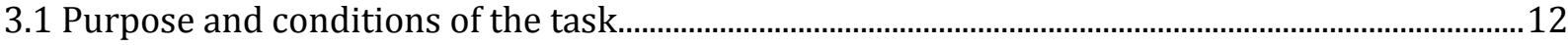

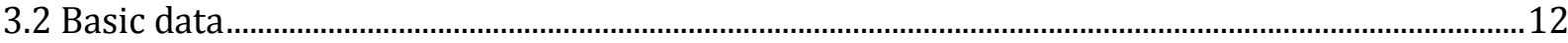

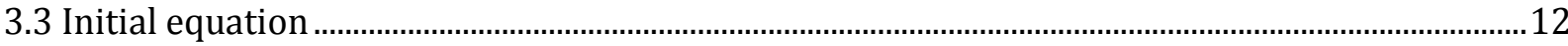

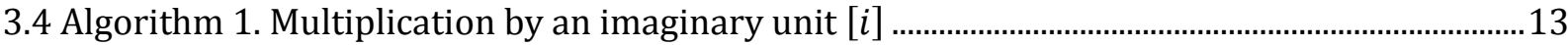

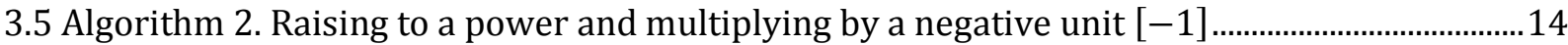

3.6 Gravity and temperature. Basic initial equation ....................................................................................

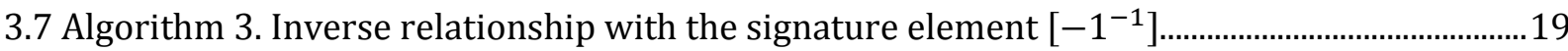

3.8 Algorithms and partial bundle of Absolute space-time ${ }^{A} S T$...........................................................22

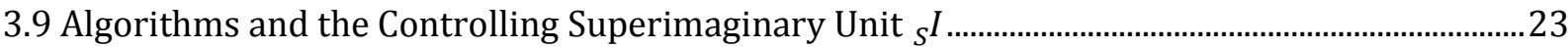

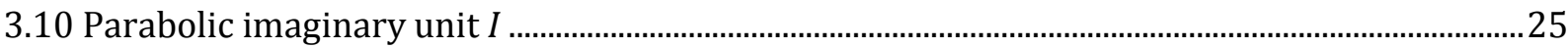

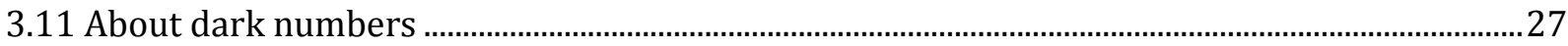

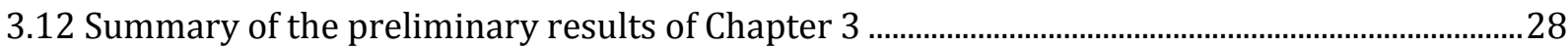

Chapter 4 Properties and characteristics of the primary (maternal) Absolute space-time ${ }^{A} S T$...........30

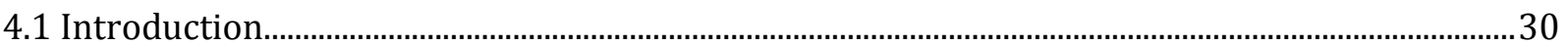

4.2 Conformity of each 0 and $\infty$. The inseparability of these concepts and meanings from each

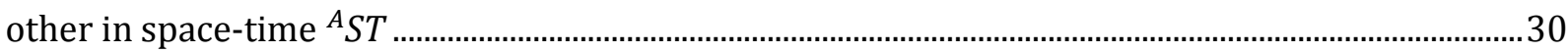

4.3 Changing the properties and characteristics of space-time ${ }^{A} S T$ depending on the period of Relaxation or from period of Big Bang to subsequent Crunch..................................................................31

4.4 Relaxation period $\left(\mathrm{Cr}_{\mathrm{n}-1}-\mathrm{BB}\right)$. The state of uncertainty of the basic parameters ....................31

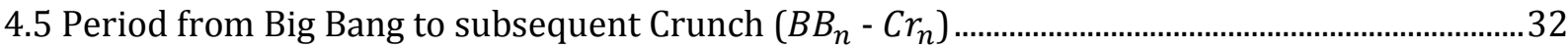

Chapter 5 Comparative properties and characteristics of space-times ${ }^{L} S T$ and ${ }^{D} S T$...........................34

5.1 Dimension of space and time ........................................................................................................... 34

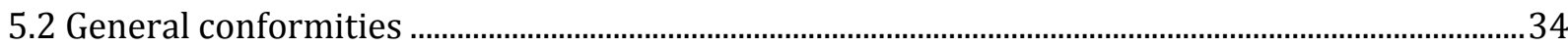

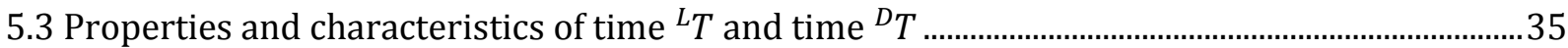

5.4 Curvature, sum of triangle angles and properties ...................................................................................

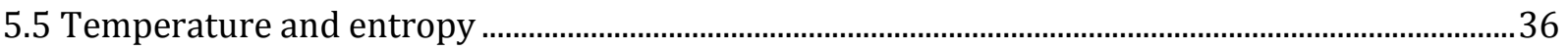




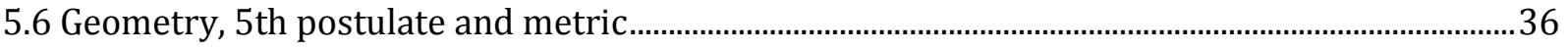

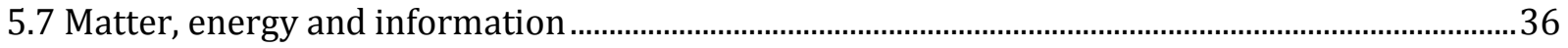

5.8 Elementary electric charge $q$ and elementary topological magnetic charge $\tilde{\theta}$...............................37

5.9 Electro-magnetic waves $(E M W)$ and positron-electric waves $(P E W)$..........................................38

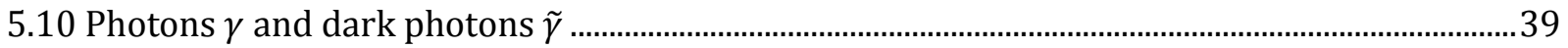

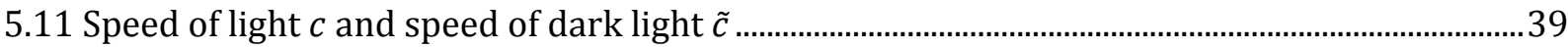

Chapter 6 Three space-time model of quantum positive gravity in ${ }^{L} S T$ and multiquantum negative

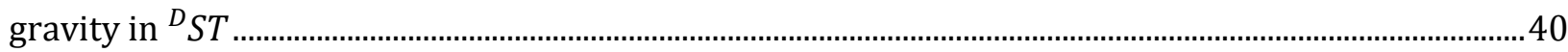

6.1 Universal gravitation laws (UGL) in ${ }^{L} S T$ and its equivalent (anti-gravity) in ${ }^{D} S T$......................40

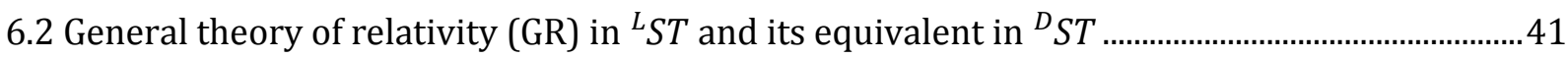

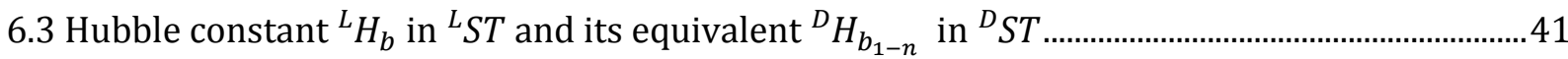

6.4 Tissues of space-times ${ }^{A} S T,{ }^{L} S T$ and ${ }^{D} S T$ as real and objective components of the Universe

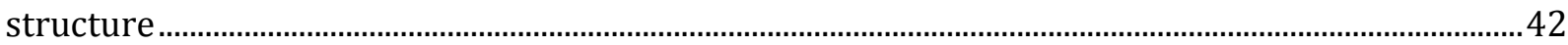

6.5 Mass formation ${ }^{L} m$ of matter particles in ${ }^{L} S T$ and mass ${ }^{D} m$ of decentralized objects of antimatter in ${ }^{D} S T$.

6.6 Self-consistent equations of the birth and evolution of space-time tissues under conditions of gravity in ${ }^{L} S T$ and antigravity in ${ }^{D} S T$.

6.7 Classification of and the new meaning of Loop Quantum Gravity ( $L Q G$ ) and String Theory (ST) based on the theory of mutually transparent self-consistent networks under conditions of gravity in ${ }^{L} S T$ and anti-gravity in ${ }^{D} S T$.

6.8 The form of matter in ${ }^{L} S T$ in conditions of gravity and the form of antimatter in ${ }^{D} S T$ in

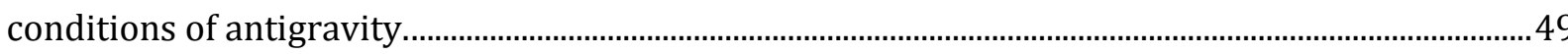

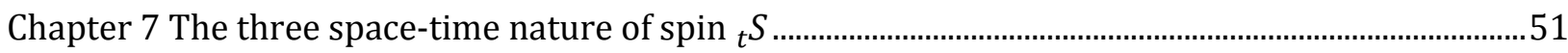

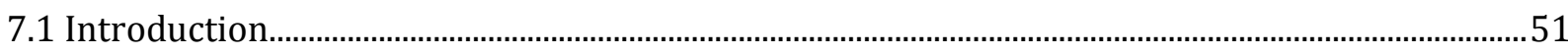

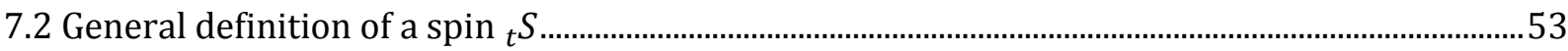

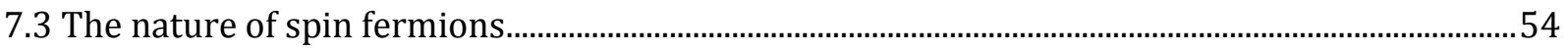

7.3.1 General positions and definition ..................................................................................................... 54

7.3.2 Geometric spin arrangement of fundamentally entangled fermions in a self-consistent atom

7.3.3 Geometric spin arrangement of fundamentally entangled free fermions during the motion (outside the atom or nucleus) along oscillating non-closed orbits......................................................57

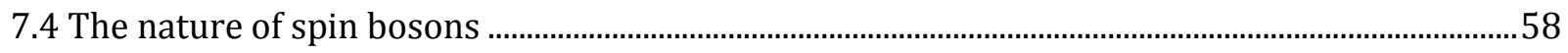

7.4.1 General positions and definition .......................................................................................... 58

7.4.2 The geometric spin position of fundamentally entangled massless bosons while moving

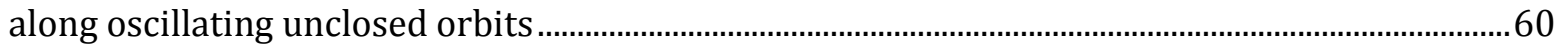

7.4.3 Spin features when fundamentally entangled massive vector ${ }^{ \pm} \mathrm{W}$ bosons are in motion.. 61 
7.4.4 Spin features of a massive vector ${ }^{0} Z$ boson. Prediction. .........................................................61

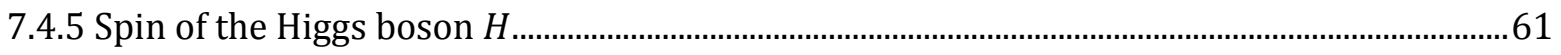

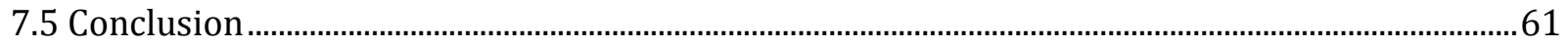

Chapter 8 A self-consistent particle-soliton model of a hydrogen atom (protium) in the three space-

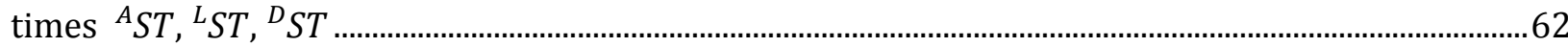

Chapter 9 Fundamental interactions in space-times ${ }^{L} S T$ and ${ }^{D} S T$....................................................64

Chapter 10 Fields and prevailing processes in space-time ${ }^{L} S T$ and ${ }^{D} S T$.............................................66

Chapter 11 Principles of motion of material objects in space-time ${ }^{L} S T$ and ${ }^{D} S T$................................67

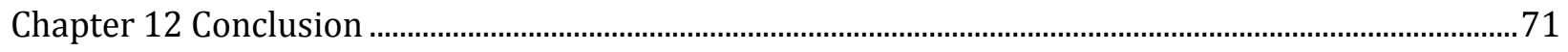

Used terms, symbols and abbreviations .................................................................................................. 75

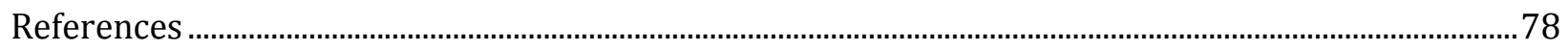

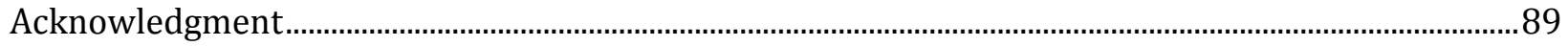




\section{Chapter 1 Introduction}

The curvature of space in the Universe, its dimension, space-time and geometric relations realized in it are the main questions of cosmology and theoretical physics. The General Theory of Relativity $(G R)[1,2]$, as the best theory of gravity, created the basis and gave these areas of research and additional impetus $[3,4,5,6,7,8,9,10,11,12,13,14,15,16,17,18$, $19,20,21,22,23,24,25,26,27,28]$. Nowadays, the Standard Model is universally recognized and is accepted as the best theory describing fundamental interactions [29]. However, in modern physics, a significant number of problems have accumulated that need to be resolved [30, 31, 32]. In addition to the above, it is primarily these problems and questions: observed baryonic asymmetry in the Universe [33,34]; Why, despite the inviolability of the second law of thermodynamics, we do not observe the thermal death of the Universe [35, 36]?; The incompleteness of the inflation model of the Big Bang $(B B)$ theory [37, 38, 39, 40, $41,42,43,44,45,46,47]$; Where has the cold stage of development of the Universe 'gone' to $[48,49,50]$ ?; Does the Universe oscillate [51, 52, 53, 54, 55, 56, 57, 58]?; Not understanding the nature of dark matter and dark energy and why the observed space continues to rapidly expand?; What is the nature of mass?; What is the fundamental role of the Brout-EnglertHiggs field and the Higgs boson?; Is the electroweak interaction theory complete?; Not understanding the origin of inertia forces; Where is the elementary magnetic charge hidden?; What are the elementary electric and elementary magnetic charges?; Not understanding the nature of spin of bosons and fermions; What is the nature of nuclear forces and quarks confinement?; What is the tunneling effect mechanism?; The lack of a complete theory of superconductivity; Why are there three generations of elementary particles?; How should the concept of a physical field be correctly defined?; What are compact massive objects observed in astronomy?; and the lack of a quantum gravity theory.

To try to answer these questions, some essential new ideas are required that lie at the very core of the $B B$ theory and allow to directly connect cosmology with both already known and not yet discovered laws of physics. Developed by the author, a three space-time (positive, negative and zero curvature) model structure of a stationary self-consistent Universe provides new ideas and wide opportunities for building a unified physical theory. This model allows to obtain six basic parameters in each space-time, which are responsible for the evolution of the Universe from $B B$ to $\mathrm{Cr}$ (see Chapter 3), and also makes clear the approach to determining the dimension of the Universe (see Chapter 12).

In cosmology, the problem of the observed baryon asymmetry and hidden mass is naturally solved, since from the moment $B B$ all the born matter develops in the space-time we observe, of positive curvature ${ }^{L} S T$, and all the born antimatter develops in the not observed by us, space-time of negative curvature ${ }^{D} S T$ (see Chapter 5 ).

The transition from the macro level to micro objects allows one to recognize the three spacetime nature of spin and develop its mechanism (see Chapter 7), which directly leads to a three space-time self-consistent particle-soliton model of the atom (see Chapter 8). This 
approach allows us to give a new interpretation of quantum mechanics, revealing the meaning of the Schrödinger and Dirac equations (see Chapter 11), and also to consistently and substantially supplement the modern quantum theory of elementary particles.

The way out of the "captivity of Heisenberg's uncertainty" becomes possible thanks to a new way of introducing generalized complex numbers (see sections 3.9, 3.10 of Chapter 3 and Chapter 4). Each imaginary unit: parabolic $I$, elliptic $i$, and hyperbolic $\tilde{\imath}$ is specified in strict accordance with the sign of the curvature of its space-time. Namely, generalized complex numbers, developing theories of functions of complex variables, each, strictly in its own space-time, play a major role in ensuring inter-space-time communication and make possible the "effect of self-consistency" in the Universe.

The author was also forced to introduce new concepts, terms and designations without which the formalization of this theory would be impossible. One of these new fundamental concepts is the Controlling Superimaginary Unit ${ }_{S} I$, which in the period from $\mathrm{Cr}$ to the next $B B$ contains imaginary: parabolic $I$, elliptic $i$ and hyperbolic $\tilde{l}$ units and, according to the author, along with the primary (maternal) Absolute space-time ${ }^{A} S T$, plays an essential role in the Universe (see Chapter 4).

The study of the possibilities of constructing a self-consistent model of three space-time quantum positive gravity in ${ }^{L} S T$ and multiquantum negative gravity in ${ }^{D} S T$ is discussed in Chapter 6. Firstly, given that one of the six basic parameters is gravity, it is possible to obtain relatively simple self-consistent formulas for determining gravity and antigravity (see section 6.6). Secondly, new understanding and definitions are received by the concepts themselves: positive gravity and negative gravity (antigravity). Thirdly, a new meaningful meaning and impulse are acquired by two complementary theories: loop quantum gravity (hereinafter referred to as $L Q G$ ) and string theory (hereinafter referred to as $S T$ ) based on the theory of mutually transparent self-consistent networks under gravity in ${ }^{L} S T$ and antigravity in ${ }^{D} S T$ (see section 6.7). Namely, the action of antigravity manages to explain the soliton nature of the form of antimatter in space-time of negative curvature ${ }^{D} S T$ (see section 6.8) and as the result, to propose the soliton-particle model of the atomic nucleus (and strong interaction, see Chapters 5 and 9).

This first publication does not attempt to study the specificities of some narrow fields of physics (which will be done in subsequent papers, including "TSSCU Formalism", "Features of Gauge Theory and CPT Theorem in TSSCU" and others) arising in this cosmological model. Instead, it evaluates the proposed hypothesis for the lack of internal inconsistency, its essential applications and prospects.

Despite the expressed debatable nature of the proposed work, the introduction of new space-time symmetry in the $B B-C r$ model and built on this basis theory of a stationary selfconsistent Universe can be used to study fundamental problems of cosmology and modern physics. 


\section{Chapter 2 TSSCU Initial provisions}

1. The Universe is one (no parallel and/or multi Universes exist).

2. The Universe consists of two substances: primary (maternal) Absolute space-time $\left({ }^{A} S T\right)$ and Controlling Superimaginary Unit ${ }_{s} I$, which unites in itself generalized complex numbers with parabolic $I$, hyperbolic $\tilde{l}$ and elliptic $i$ imaginary units (see section 3.5, 3.9, 3.10 of Chapter 3 and section 4.2 of Chapter 4). The primary (maternal) Absolute spacetime ${ }^{A} S T$ and Controlling Superimaginary Unit ${ }_{s} I$ periodically interact with each other (Figure 7) forming a generalized Paracomplex Absolute space-time ${ }_{I}^{A} S T$ with imaginary parabolic unit $I$, in which Big Bang $(B B)$ takes place with subsequent inevitable Crunch $(\mathrm{Cr})$. The period when both substances stop interacting with each other (from the moment of $\mathrm{Cr}$ to the subsequent $B B$ ) is the period of Relaxation.

3. At the moment of $B B$ (Figure 1) in generalized Paracomplex Absolute space-time ${ }_{1}^{A} S T$ (that includes primary (maternal) ${ }^{A} S T$ with zero $(\Omega=0)$ and infinity $(\Omega=$ $\infty)$ curvatures at the same time (see section 2 of Chapter 4 ), with Euclidean geometry $(\varepsilon)$ when interacting with sphere with distance-radius and curvature, which are equal to imaginary parabolic unit $I\left(S_{s p h}=I, \Omega_{s p h}=I\right)$ ) space-time symmetry is realized. Synchronously, that is in the synchronization time $\left(T_{\text {synch }}\right)$, two mutually transparent space-times are born:

- Hot space-time $\left[{ }^{L} S T\right.$, Light (Real) Space - Time] of constant positive curvature $\left({ }^{L} \omega>0\right)$ with positive density of matter ${ }^{L} M$ and energy ${ }^{L} E$, stochastic information ${ }^{L} I$, in which the elliptic Riemannian geometry $(\mathcal{R})$ is realized, and the tissue of space homogenously expands (relative to the tissue of Absolute space-time ${ }^{A} S T$ ) in time ${ }^{L} T$

- Cold space-time $\left[{ }^{D} S T\right.$, Dark (Hidden) Space - Time] of constant negative curvature $\left({ }^{D} \omega<0\right)$, with negative density of matter ${ }^{D} M$ and energy ${ }^{D} E$, fractal (or comparable, this term requires an exact scientific definition) information ${ }^{D} I$, in which the hyperbolic Lobachevsky-Bolyai $(\mathcal{L B})$ geometry is realized, and the tissue of space heterogeneously contracts (relative to the tissue of Absolute space-time ${ }^{A} S T$ ) in time ${ }^{D} T$

Space-times ${ }^{L} S T$ and ${ }^{D} S T$ have the following properties:

- They are mutually transparent, that is, they do not have the ability to directly interact with each other

- They are mutually self-consistent, as they are forced to purposefully interact with each other through the generalized Paracomplex Absolute space-time ${ }_{I}^{A} S T$ (see sections 3.9 and 3.10 of Chapter 3)

- They are fundamentally entangled to each other, that is, completely interdependent (see section 2.3 of Chapter 2, "The concept of fundamental entanglement - $\bar{æ}$ ")

- They are complete antipodes of each other and have antagonistic properties

- They are quantized, i.e., fundamental interactions in both space-times possess quants of actions 
4. Space-times ${ }^{L} S T$ and ${ }^{D} S T$ consist of matter and antimatter tissues (special field-forms), which are developing in time ${ }^{L} T$ and ${ }^{D} T$, accordingly.

5. In all three space-times ${ }^{A} S T,{ }^{L} S T$ and ${ }^{D} S T$, the basic categories are: Matter, Energy, and Information.

6. At any moment of $T_{\text {synch }}$ the total mass density and energy density of the two space-times ${ }^{L} S T$ and ${ }^{D} S T$ is equal to zero.

7. Stochastic information ${ }^{L} I$ in ${ }^{L} S T$ and fractal information ${ }^{D} I$ in ${ }^{D} S T$ are mutually exclusive (antipodes of each other) and, when $\mathrm{Cr}$ happens in $T_{\text {synch }}$ they mutually neutralize each other out.

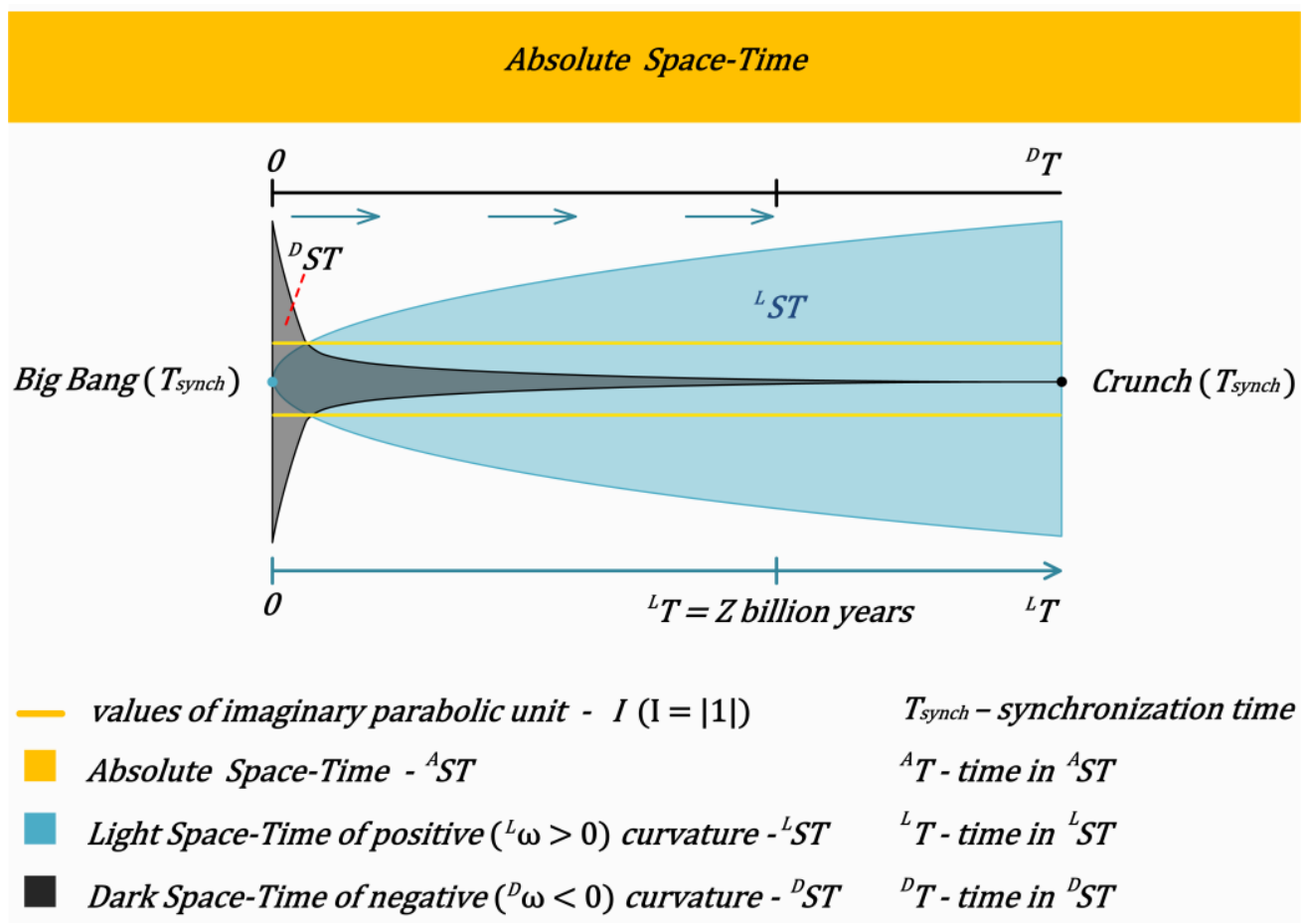

Figure. 1. Three space-time model of the structure of the Universe. In the generalized Paracomplex Absolute space-time ${ }_{I}^{A} S T$, in the moment of the Big Bang synchronously (in $T_{\text {synch }}$ ), two mutually transparent self-consistent non-commutative space-times are born: constant positive curvature ${ }^{L} S T$ and constant negative curvature ${ }^{D} S T$. At any point in time of synchronization $T_{\text {synch }}$ the product of curvature ${ }^{L} \omega$ of spacetime ${ }^{L} S T$ on curvature ${ }^{D} \omega$ of space-time ${ }^{D} S T$ is equal to Imaginary Parabolic unit $I$ : $\left[{ }^{L} \omega\left(T_{\text {synch }}\right) \cdot{ }^{D} \omega\left(T_{\text {synch }}\right)=I\right]$, where $I=|\mathbf{1}|$. The fate of both space-times ${ }^{L} S T$ and ${ }^{D} S T$ is predetermined, and they will also disappear synchronously (in $T_{\text {synch }}$ ) at the moment of Crunch.

\subsection{The concept of time synchronization $T_{\text {synch }}$}

On one hand, the spaces ${ }^{L} S T$ and ${ }^{D} S T$ have their own times ${ }^{L} T$ and ${ }^{D} T$, with their own tempo and different properties. But; on the other hand, both ${ }^{L} T$ and ${ }^{D} T$ times are generated from primary eternal time ${ }^{A} T$, by the process of $B B$ initiation, (see Chapters 3 and 4 ). As a result, 
all fundamentally entangled processes in these two spaces will proceed synchronously in time, referred to as time synchronization $T_{\text {synch }}$.

\subsection{The concept of interdimensional potential ${ }^{S T} U$}

At $T_{\text {synch }}$ time, in the moment of $B B$, the following were formed: space-time ${ }^{L} S T$ with a very high initial temperature and cold space-time ${ }^{D} S T$, probably with a temperature equivalent to absolute zero (0K). Considering the temperature difference at the moment of birth of both space-times ${ }^{L} S T$ and ${ }^{D} S T$, as well as their antagonistic properties, in the generalized Paracomplex Absolute space-time ${ }_{I}^{A} S T$ an interdimensional potential ${ }^{S T} U$ arises, enabling all processes from $B B$ to $C r$ to be realized.

\subsection{The concept of fundamental entanglement ææ}

The following is an abbreviated definition of the concept of "fundamental entanglement". A full and detailed definition will be given in the next article "TSSCU Formalism".

The fundamental entanglement is $\bar{\not}$, (not to be confused with the quantum entanglement of two or more objects in space-time ${ }^{L} S T$, introduced in 1935 by Einstein et al. [59], which is a special (particular) quickly disappearing case of fundamental entanglement), a property of paired objects born at $T_{\text {synch }}$ in space-times ${ }^{L} S T$ and ${ }^{D} S T$ (each object is born in its own space-time) that maintain a full dependence on each other until transformation into other objects or disappearance, which are bound to occur, also in $T_{\text {synch }}$. 


\section{Chapter 3 Primary (maternal) Absolute space-time ${ }^{A} S T$ as the basis for the Big Bang $(B B)$ model and subsequent Crunch $(\mathrm{Cr})$}

\subsection{Purpose and conditions of the task}

It is necessary to search for algorithms (albeit, at first, incomplete, but simplest) that allow simulating the bundle of the primary (maternal) generalized Absolute space-time ${ }^{A} S T$ into two space-times ${ }^{L} S T$ and ${ }^{D} S T$. This should be focused on stationarity of ${ }^{A} S T$ and complete preservation of its integrity and indifference (self-consistency condition) in any processes occurring in ${ }^{L} S T$ and ${ }^{D} S T$, and at any moment $T_{\text {synch }}$ from the moment of $B B$ to $C r$.

For clarity purposes, upper case letters will be used in formulas in ${ }^{A} S T$ letters (except for coordinates), and lower case letters will be used in ${ }^{L} S T$ and ${ }^{D} S T$ (except for temperature and gravity). In cases, where the imaginary parabolic unit denoted as $I$, is represented by a numerical value, it will be denoted as 1 in bold.

\subsection{Basic data}

In ${ }^{A} S T$, Euclidean geometry $(\mathcal{E})$ is realized. The metric in Euclidean geometry is represented by the following equation:

$$
S^{2}=\left(d x^{2}+d y^{2}+d z^{2}\right)
$$

Or, accordingly:

$$
S=\sqrt{d x^{2}+d y^{2}+d z^{2}}
$$

where, $S$ is the distance or radius of the sphere.

Taking into account equation 2, connecting distance, velocity and time:

$$
S=V \cdot T
$$

Leading to equation 3 :

$$
\sqrt{d x^{2}+d y^{2}+d z^{2}}=V \cdot T
$$

\subsection{Initial equation}

In order to obtain space-times ${ }^{L} S T$ and ${ }^{D} S T$, the constant positive curvature and constant negative curvature, the parameter of curvature of a sphere $\Omega$ with a radius $S$ needs to be added to the left side of equation 3 , that is equal to:

$$
\Omega=\frac{1}{S^{2}}
$$

Taking into consideration equation 4 , it is obvious that the simplest and the most acceptable solution, suitable for the designated goals, while allowing to introduce into equation 3 parameter $\Omega$, is the sphere of unit radius, i.e. $S=1$ and accordingly $\Omega=1, V=1, T=1$. 
Equation 3 supplemented with parameter $\Omega$ :

$$
\Omega \cdot \sqrt{d x^{2}+d y^{2}+d z^{2}}=V \cdot T
$$

where, $\Omega=1, S=1, V=1, T=1$.

Equation 5 can serve as the starting point for finding the right algorithm for bundle of ${ }^{A} S T$ and further enabling necessary mathematical operations. Each of the algorithms discussed below may have one or more signatures. A "signature" represents a set of algebraic and other operations that must be performed with an equation describing a space-time metric (or with a more extended equation), resulting in a "set of properties" of the primary maternal spacetime ${ }^{A} S T$ and newly born space-times ${ }^{L} S T$ and ${ }^{D} S T$, that cannot be cancelled (they are given at the time of bundle), and that determine the conditions for the development and interaction of all three space-times, starting from the moment $B B$ to $C r$.

\subsection{Algorithm 1. Multiplication by an imaginary unit [i]}

The first step was to find out whether ${ }^{D} S T$ can consist of imaginary coordinates and imaginary time and meet the requirements presented in Chapter 2. Despite the obvious negative answer, simple operations were carried out to prevent this question in the future. To enable this, the signature $\left[\cdot\left(1^{2}\right), \cdot(i), \leftrightarrow\right]$ was considered, where $\leftrightarrow$ means bundle.

Each factor on the left and right sides of equation 5 was raised to the power of 2 , and then multiplied by $i$, as follows:

$$
\left(i \Omega^{2}\right) \cdot\left(i S^{2}\right)=\left(i V^{2}\right) \cdot\left(i T^{2}\right)
$$

Equation 6 can also be represented in the following form:

$$
\Omega \cdot(i \Omega) \cdot S \cdot(i S)=V \cdot(i V) \cdot T \cdot(i T)
$$

The bundle operation of equation 7 represented in the form of two non-commutative equations 8L and 8D and expressed in time:

Space-time ${ }^{L} S T$ :

$$
t=\frac{\omega \cdot \sqrt{\left(d x^{2}+d y^{2}+d z^{2}\right)}}{v}
$$

where, $t>0, \omega>0, v>0, s>0$

Space-time ${ }^{D} S T$, :

$$
i t=\frac{i \omega \cdot i \sqrt{\left(d x^{2}+d y^{2}+d z^{2}\right)}}{i v}
$$

The resulting equation is space-time representation of positive curvature in ${ }^{L} S T$ with 3 real coordinates and 1 time (4 dimensions), and in ${ }^{D} S T$, an imaginary coordinate (the value of which depends on 3 real coordinates) with imaginary time. Evidently, the result is not the 
full-fledged (4 dimensions) of space-time ${ }^{D} S T$, as declared in Chapter 2; therefore, the signature $\left[\cdot\left(1^{2}\right), \cdot(i), \leftrightarrow\right]$ is incorrect.

Considering the signature $\left[\cdot\left(1^{4}\right), \cdot(i), \leftrightarrow\right]$, each factor on the left and right sides of equation 5 was raised to the power of 4 , and then multiplied by $i$, as follows:

$$
\left(i \Omega^{4}\right) \cdot\left(i S^{4}\right)=\left(i V^{4}\right) \cdot\left(i T^{4}\right)
$$

Equation 9 can also be represented in the following form:

$$
\Omega^{2} \cdot\left(i \Omega^{2}\right) \cdot S^{2} \cdot\left(i S^{2}\right)=V^{2} \cdot\left(i V^{2}\right) \cdot T^{2} \cdot\left(i T^{2}\right)
$$

The bundle operation of equation 10 represented in the form of two non-commutative equations 11L and 11D and expressed in time:

Space-time ${ }^{L} S T$ :

$$
t^{2}=\frac{\omega^{2} \cdot\left(d x^{2}+d y^{2}+d z^{2}\right)}{v^{2}}
$$

where, $t>0, \omega>0, v>0, s>0$

Space-time ${ }^{D} S T$ :

$$
i t^{2}=\frac{i \omega^{2} \cdot\left(i d x^{2}+i d y^{2}+i d z^{2}\right)}{i v^{2}}
$$

The resulting equation is space-time of positive curvature ${ }^{L} S T$ with 3 real coordinates and 1 time (4 dimensions), and some space-time with 3 imaginary coordinates and 1 imaginary time. In this case, the accuracy of purely imaginary coordinates with imaginary time were not even assessed, since the obtained equations 11L and 11D contradict points (3-7) of the initial provisions of Chapter 2 , and therefore the applied signature $\left[\cdot\left(1^{4}\right), \cdot(i), \leftrightarrow\right]$ is also incorrect.

Conclusion: Space-time ${ }^{D} S T$ is not a space with imaginary coordinates and imaginary time, and algorithm 1 is inoperative.

\subsection{Algorithm 2. Raising to a power and multiplying by a negative unit $[-1]$}

Considering the signature $\left[\cdot 1^{2}, \cdot(-1), \leftrightarrow\right]$, each factor on the left and right sides of equation 5 was raised to the power of 2 and then multiplied by $(-1)$ as follows:

$$
\left(-\Omega^{2}\right) \cdot\left(-S^{2}\right)=\left(-V^{2}\right) \cdot\left(-T^{2}\right)
$$

Equation 12 can also be represented in the following form:

$$
\Omega \cdot(-\Omega) \cdot S \cdot(-S)=V \cdot(-V) \cdot T \cdot(-T)
$$

The bundle operation of equation 13, represented in the form of two self-consistent noncommutative equations $14 \mathrm{~L}$ and $14 \mathrm{D}$ and expressed in time: 
Space-time ${ }^{L} S T$ :

$$
t=\frac{\omega \cdot \sqrt{d x^{2}+d y^{2}+d z^{2}}}{v}
$$

where, $t>0, \omega>0, s>0, v>0$

Space-time ${ }^{D} S T$ :

$$
-t=\frac{-\omega \cdot-\sqrt{d x^{2}+d y^{2}+d z^{2}}}{-v}
$$

At a first glance, equations 14L and 14D are satisfactory and determine parameters, as well as some characteristics, of the further development of space-times of positive and negative curvatures, respectively. The only factor not completely determined is: $-\sqrt{d x^{2}+d y^{2}+d z^{2}}$ , that is included in equation 14D. Next, to understand how a coordinate system can be represented in the space-time ${ }^{D} S T$, the following two options should be considered. The first option with positive-negative symmetry of coordinate values is shown in Figure 2, represents the author's interpretation of the work of Feynman [60], that indirectly characterizes some properties of ${ }^{D} S T$. The second option, with positive-negative symmetry of the directions of the coordinate axes is shown in Figure 3.
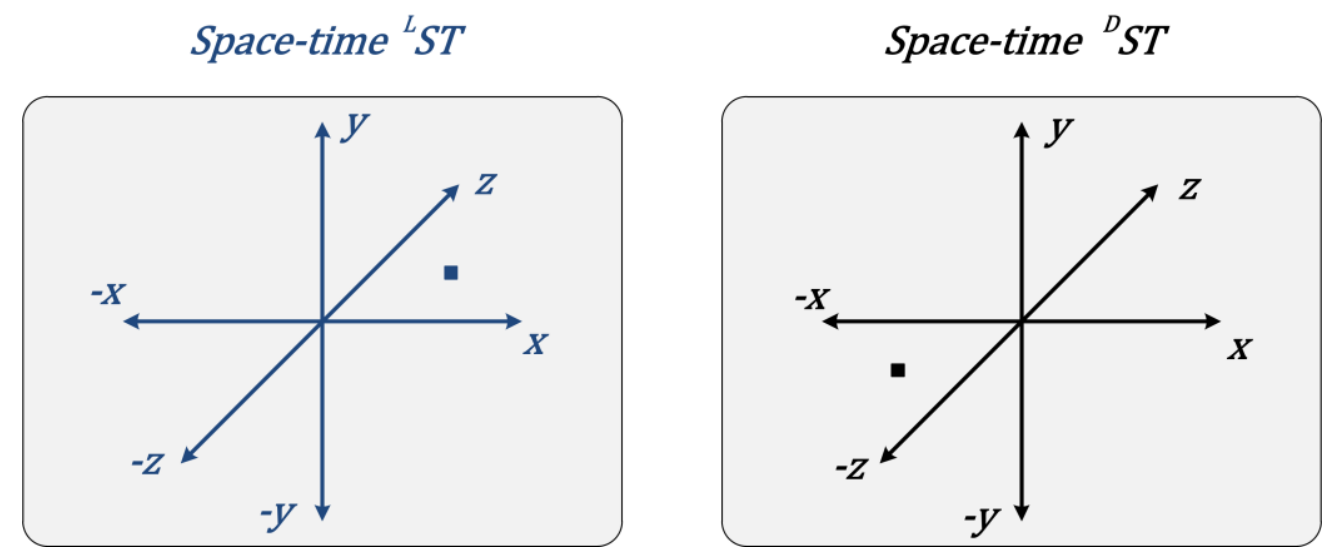

Figure 2. Comparison of rectangular (Cartesian) coordinate systems in space-time ${ }^{L} S T$ and ${ }^{D} \boldsymbol{S T}$ according to the first option. On the left is a common mathematical rectangular coordinate system in space-time ${ }^{L} S T$. On the right is a hypothetical rectangular coordinate system in space-time ${ }^{D} S T$, in which the positive coordinate axes in ${ }^{L} S T$ and ${ }^{D} S T$ as well as the negative coordinate axes in ${ }^{L} S T$ and ${ }^{D} S T$ are directed in the same route (coincide). 
Space-time ${ }^{L} S T$

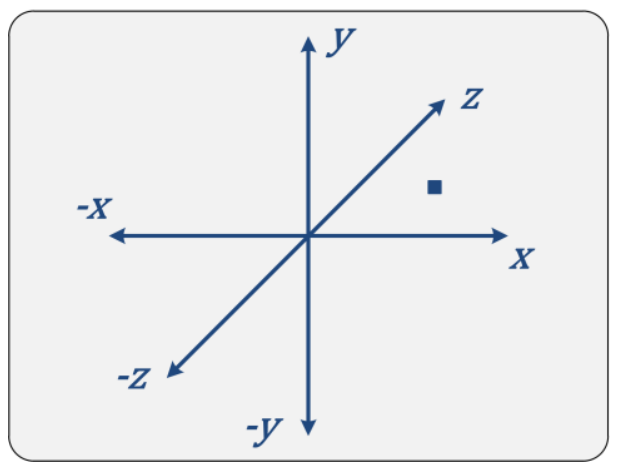

Space-time ${ }^{D} S T$

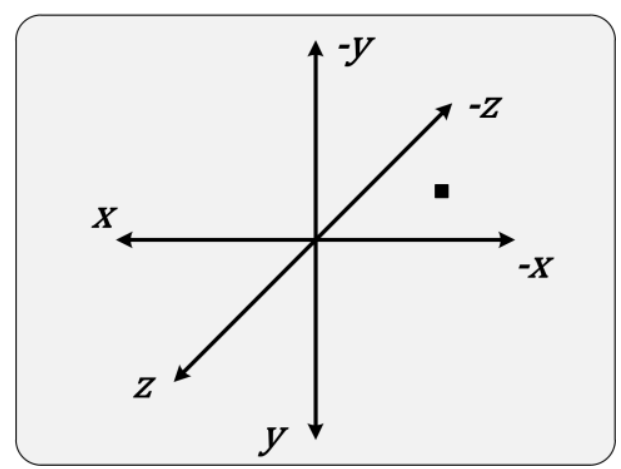

Figure 3. Comparison of rectangular (Cartesian) coordinate systems in space-time ${ }^{L} S T$ and ${ }^{D} \boldsymbol{S T}$ according to the second option. On the left is a common mathematical rectangular coordinate system in space-time ${ }^{L} S T$. On the right is a hypothetical rectangular coordinate system in space-time ${ }^{D} S T$, in which the positive coordinate axes in ${ }^{L} S T$ and ${ }^{D} S T$ as well as the negative coordinate axes in ${ }^{L} S T$ and ${ }^{D} S T$ are directed in the opposite routes.

Taking into account that space-times ${ }^{L} S T$ and ${ }^{D} S T$ are complete antipodes of each other (according to section 3 of Chapter 2), the option with positive-negative symmetry of the direction of the coordinate axes which is shown in Figure 3 can be assumed to fit the goal better. Indeed, this option, with positive-negative symmetry of the direction of the coordinate axes, was applied when creating Figure 1.

To deal with equations $14 \mathrm{~L}$ and $14 \mathrm{D}$, it is necessary to note that, when in bundle ${ }^{A} S T$, spacetime ${ }^{L} S T$ delegates (by default) the familiar signature element $\left(+1^{+1}\right)$, which means (plus one in degree plus one), referred to as an absolute dominant plus and indicated in signatures for further research. In space-time ${ }^{D} S T$, an element of signature $(-1)$ is delegated, referred to a dominant minus.

Based on the above, in addition to the traditional algebra with absolute dominant plus applied in ${ }^{L} S T$, a positive-negative symmetric algebra with a dominant minus in space-time ${ }^{D} S T$ can be introduced. To do so, corresponding rules of operations of addition, multiplication, and other elements were set to be used for algebra of "dark" numbers (not to be confused with regular negative numbers, these were denoted by the first two Latin letters: $D a)$ in space-time ${ }^{D} S T$. The rules of operations for the set of real numbers $(\mathbb{R})$ in ${ }^{L} S T$ and the set of "dark" numbers (D) in ${ }^{D} S T$ are compared in Table 1. 
Table 1. Comparison of the rules of mathematical operations for real numbers $(R e)$, obtained using the signature element $\left[+1^{+1}\right]$ in ${ }^{L} S T$, and dark numbers $(D a)$, obtained using the signature element $[-1]$ in ${ }^{D} S T$.

\begin{tabular}{|c|c|}
\hline Space-time ${ }^{L} S T$ & Space-time ${ }^{D} S T$ \\
\hline Signature element $\left[+1^{+1}\right]$ & Signature element $[-1]$ \\
\hline Real numbers $(R e): a, b, c, d \ldots$ & Dark numbers $(D a):-\widetilde{a},-\widetilde{b},-\widetilde{c,}-\tilde{d} \ldots$ \\
\hline$+a>0>-a$ & $-\tilde{a}>0>+\tilde{a}$ \\
\hline $\begin{array}{l}+a+b=+c \\
-a+b= \pm c\end{array}$ & $\begin{array}{l}-\tilde{a}-\tilde{b}=-\tilde{c} \\
+\tilde{a}-\tilde{b}= \pm \tilde{c}\end{array}$ \\
\hline $\begin{array}{l}\text { Multiplication: } \\
\qquad \begin{array}{r}(+a)(+b)=+c \\
(+a)(-b)=-c \\
(-a)(-b)=+c\end{array}\end{array}$ & $\begin{array}{l}\text { Multiplication: } \\
\qquad \begin{aligned}(-\tilde{a})(-\tilde{b})=-\tilde{c} \\
(-\tilde{a})(+\tilde{b})=+\tilde{c} \\
(+\tilde{a})(+\tilde{b})=-\tilde{c}\end{aligned}\end{array}$ \\
\hline $\begin{array}{l}\text { Raising to a power: } \\
\qquad \pm a^{2}=+c\end{array}$ & $\begin{array}{l}\text { Raising to a power: } \\
\qquad \pm \tilde{a}^{2}=-\tilde{c}\end{array}$ \\
\hline $\begin{array}{l}\text { Square root extraction: } \\
\qquad \sqrt{+a}=+c\end{array}$ & $\begin{array}{l}\text { Square root extraction: } \\
\qquad \sqrt{-\tilde{a}}=-\tilde{c}\end{array}$ \\
\hline $\begin{array}{l}\text { Complex numbers: } \\
\qquad \begin{array}{r}a+i b \\
i^{2}=-1\end{array}\end{array}$ & $\begin{array}{l}\text { Dark complex numbers: } \\
\qquad-\tilde{a}-\tilde{\imath} \tilde{b} \\
\tilde{\imath}^{2}=1\end{array}$ \\
\hline $\begin{array}{l}\text { Quaternions: } \\
\qquad \begin{array}{l}q=a+b i+c j+d k \\
\qquad i^{2}=j^{2}=k^{2}=i j k=-1\end{array} \\
\text { and so on... }\end{array}$ & $\begin{array}{l}\text { Dark quaternions: } \\
\qquad-\tilde{q}=-\tilde{a}-\tilde{b} \tilde{\imath}-\tilde{c} \tilde{j}-\tilde{d} \tilde{k} \\
\qquad \tilde{l}^{2}=\tilde{J}^{2}=\tilde{k}^{2}=\tilde{\imath} \tilde{j}=1 \\
\text { and so on... }\end{array}$ \\
\hline
\end{tabular}

Assuming that algebra with a dominant minus is realized in ${ }^{D} S T$, the origin of the imaginary elliptical unit $i$ becomes clear (revealed) from Table 1 , and its $i^{2}=-1$ in the theory of functions of a complex variable (TFCV). This multiplication is fully consistent with the rules of multiplying dark numbers in ${ }^{D} S T$. Given these findings, a new preliminary interpretation can be made that is an expanded definition of TFCV. This is a convenient and powerful mathematical tool that provides the ability to quickly and efficiently solve specific class of problems in ${ }^{L} S T$. But, at the same time, TFCV is also a local and limited "bridge-model" combining the properties of the regular algebra with absolute dominant plus and algebra with dominant minus. Such a local union based on a set of complex numbers $(\mathbb{C})$ allows to describe some aspects of the interactions of objects in space-times ${ }^{L} S T$ and ${ }^{D} S T$ (for example, Schrödinger's equation, see Chapter 11). Therefore, complex numbers include both real 
numbers and imaginary ones, and are endowed with the properties of dark numbers in ${ }^{D} S T$. As can be seen on the right column of Table 1 , in ${ }^{D} S T$, the same logic can be followed to introduce a dark imaginary hyperbolic unit $\tilde{\imath}$, whose square is equal, $\tilde{\imath}^{2}=1$, dark complex numbers, etc. The nature of complex numbers will be discussed in section 3.9 of this chapter, when discussing the undefined factor: $-\sqrt{d x^{2}+d y^{2}+d z^{2}}$. It can be written in algebra with dominant minus as: $\sqrt{-d x^{2}-d y^{2}-d z^{2}}$, since the square root of a negative number will be a negative number. Accordingly, equation $14 \mathrm{D}$ can be rewritten as:

$$
-t=\frac{-\omega \cdot \sqrt{-d x^{2}-d y^{2}-d z^{2}}}{-v}
$$

with the parameters $-t,-\omega,-s,-v>0$ in ${ }^{D} S T$.

Conclusion: algorithm 2 with a signature $\left[\cdot 1^{2}, \cdot\left(+1^{+1}\right), \cdot(-1), \leftrightarrow\right]$ is, based on a first approximation, satisfactory and requires further research, and equation $14 \mathrm{D}$ relates to algebra with dominant minus.

\subsection{Gravity and temperature. Basic initial equation}

Additional two parameters are necessary for equation 5: gravity $G_{r}$ and temperature $K$. Given the specificity of this equation, both parameters in ${ }^{A} S T$ with the Euclidean geometry $(\mathcal{E})$ at the moment of $B B$ and before $\mathrm{Cr}$ also must equal to 1 .

Gravity ${ }^{A} G_{r}=1$

Temperature ${ }^{A} K=1$

The next step would be to determine in which part (left or right) of equation 5 both parameters could be placed, taking into account the starting conditions and the dynamics of the development of space-times ${ }^{L} S T$ and ${ }^{D} S T$ (Figure 1).

In ${ }^{L} S T$, gravity $G_{r}$ represents a force that arises as opposing (tending to compensate) to the expansion of this space, and, accordingly, to a change in two inverse-dependent parameters: the distance-radius of curvature ${ }^{L} S$ upward, and the curvature ${ }^{L} \omega$ downward. Therefore, the parameters ${ }^{L} S$ and ${ }^{L} G_{r}$ are directly dependent on each other: the larger the distance-radius of curvature ${ }^{L} S$ is, the greater the gravity ${ }^{L} G_{r}$, and conversely, the greater the gravity ${ }^{L} G_{r}$ is, the greater the distance-radius of curvature ${ }^{L} S$. Evidently, in this case, the parameter $G_{r}$ must be placed on the right side of equation 5 .

The temperature ${ }^{L} K_{1}$ at the first moment $B B$ is maximal in space-time ${ }^{L} S T$, while the parameter ${ }^{L} S_{1}$ is minimal. But in space-time ${ }^{D} S T$, the opposite is true. For the second criterion: the higher the temperature $K$ in ${ }^{L} S T$ is, the greater should velocity $V$ be. Therefore, parameter $K$ must be placed on the left side of equation 5 .

As a result, for ${ }^{A} S T$, we obtain equation 15 :

$$
K \cdot \Omega \cdot S=V \cdot T \cdot G_{r}
$$


where $K$ is the temperature, $\Omega$ is the curvature of space, $S$ is the distance or radius of sphere, $V$ is velocity, $T$ is time and $G_{r}$ is gravity.

Equation 15 can serve as the main initial equation for carrying out the necessary further mathematical operations on the bundle ${ }^{A} S T$.

Performing similar operations on Equation 15 as in section 3.5 with a signature $\left[\cdot 1^{2}, \cdot\left(+1^{+1}\right), \cdot(-1), \leftrightarrow\right]$, while taking into account three factors on the left and three on the right, leads to the two self-consistent non-commutative equations $16 \mathrm{~L}$ and 16D, each determining the initial conditions and some dynamic characteristics of the further development of spaces-times of positive and negative curvature, respectively.

Space-time ${ }^{L} S T$ :

$$
t=\frac{K \cdot \omega \cdot \sqrt{d x^{2}+d y^{2}+d z^{2}}}{G_{r} v}
$$

where, $t>0, K>0, \omega>0, s>0, G_{r}>0, v>0$

In the obtained 16L equation, the familiar positive time ${ }^{L} t$ has, in addition to a predicted inverse dependence on gravity $G_{r}$ and velocity $v[61,62,63,64,65,66]$, a direct dependence on temperature $K$, space curvature $\omega$ and distance-radius $s$.

Space-time ${ }^{D} S T$ :

$$
-t=\frac{(-K) \cdot(-\omega) \cdot \sqrt{-d x^{2}-d y^{2}-d z^{2}}}{\left(-G_{r}\right) \cdot(-v)}
$$

where, $-t>0,-K>0,-\omega>0,-s>0,-G_{r}>0,-v>0$. Equation 16 relates to algebra with dominant minus.

Conclusion: the resulting equation 15 is the main initial equation and requires further research.

\subsection{Algorithm 3. Inverse relationship with the signature element $\left[-1^{-1}\right]$}

To achieve the goals set above, the possibility of implementing an algorithm with an inverse relationship with a signature $\left[\cdot 1^{2}, \cdot\left(+1^{+1}\right), \cdot\left(-1^{-1}\right), \leftrightarrow\right]$ needs to be considered. The signature element $\left(-1^{-1}\right)$ will be referred to as the absolute dominant minus (minus one in degree minus one). The left and right side of equation 15, will be raised to the power of 2 and presented in the following form:

$$
K \cdot(K) \cdot \Omega \cdot(\Omega) \cdot S \cdot(S)=V \cdot(V) \cdot T \cdot(T) \cdot G_{r} \cdot\left(G_{r}\right)
$$

Each factor of the left and right sides in brackets was multiplied by $\left(-1^{-1}\right)$. As a result, the following equation is obtained:

$$
K \cdot\left(-\frac{1}{K}\right) \cdot \Omega \cdot\left(-\frac{1}{\Omega}\right) \cdot S \cdot\left(-\frac{1}{S}\right)=V \cdot\left(-\frac{1}{V}\right) \cdot T \cdot\left(-\frac{1}{T}\right) \cdot G_{r} \cdot\left(-\frac{1}{G_{r}}\right)
$$


The bundle operation of equation 18, represented in the form of two self-consistent noncommutative equations $19 \mathrm{~L}$ and $19 \mathrm{D}$ and expressed in time:

Space-time ${ }^{L} S T$ :

$$
t=\frac{K \cdot \omega \cdot\left(d x^{2}+d y^{2}+d z^{2}\right)}{G_{r} \cdot v}
$$

where, $t>0, K>0, \omega>0, s>0, G_{r}>0, v>0$

Space-time ${ }^{D} S T$ :

$$
-\frac{1}{t}=\frac{\left(-\frac{1}{K}\right) \cdot\left(-\frac{1}{\omega}\right) \cdot \frac{-1}{\sqrt{-d x^{2}-d y^{2}-d z^{2}}}}{\left(-\frac{1}{G_{r}}\right) \cdot\left(-\frac{1}{v}\right)}
$$

Or equation 19D could be written in a more of a compact form:

$$
-t^{-1}=\frac{\left(-K^{-1}\right) \cdot\left(-\omega^{-1}\right) \cdot\left(-s^{-1}\right)}{\left(-G_{r}^{-1}\right) \cdot\left(-v^{-1}\right)}
$$

where, $-t^{-1}>0,-K^{-1}>0,-\omega^{-1}>0,-s^{-1}>0,-G_{r}^{-1}>0,-v^{-1}>0$, and equations 19D (or 20D) relate to algebra with an absolute dominant minus.

The results are analyzed using Figure 4 and Table 2.

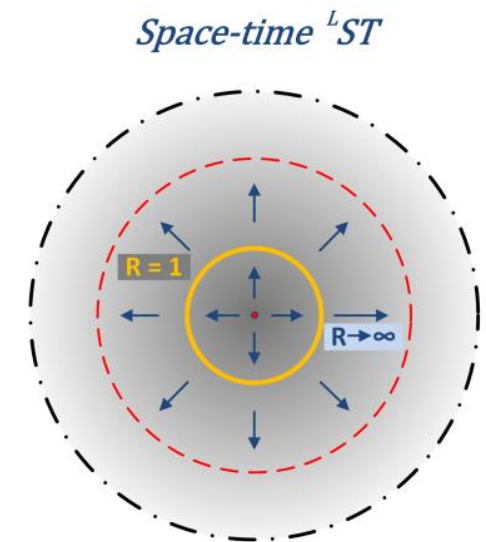

Unit radius sphere $-{ }^{A} S T$

Light Space-Time $-{ }^{L} S T$

Dark Space-Time - ${ }^{D} S T$
Space-time ${ }^{D} S T$

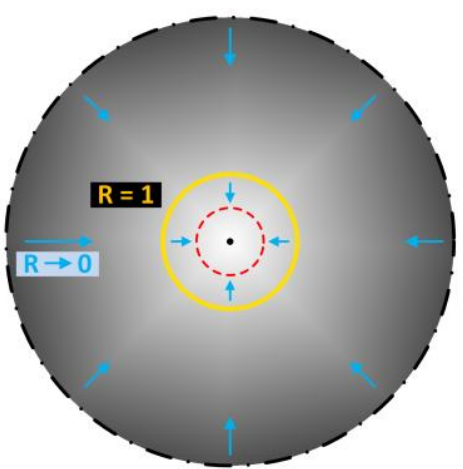

- Zero neighborhood

?. Infinity

(-) Modern stage of the Universe development

Figure 4. The flat generalized model-diagram of the Big Bang, and subsequent Crunch, in cross section, which complements the model-diagram shown in Figure1. 
Table 2. Comparison of properties of space-time ${ }^{L} S T$ and ${ }^{D} S T$ at the moment of their birth $(B B)$, stages of development and subsequent Crunch $(\mathrm{Cr})$ taking into account inverse relationship of both space-times between themselves.

\begin{tabular}{|c|c|}
\hline Space-time ${ }^{L} S T$ & Space-time ${ }^{D} S T$ \\
\hline $\begin{array}{l}B B \text { begins in a radius other than zero } \\
\left({ }^{L} r>0\right) \text { by an infinitesimal value and } \\
\text { extends outward (expansion) in all } \\
\text { directions, approaching a unit radius } \\
\left({ }^{L} r \approx 1\right) \text {, and then tends to a radius } \\
\left.\text { tending to infinity ( }{ }^{L} r \rightarrow \infty\right) \text { to } C r \text {. } \\
\text { As explained below (see section } 3.10 \text { ), the } \\
\text { value of }{ }^{L} r=1 \text { synchronous with value } \\
{ }^{D} r=-1 \text { in }{ }^{D} S T \text { is impossible. }\end{array}$ & $\begin{array}{l}B B \text { begins in a radius other than infinity } \\
\left({ }^{D} r<-\infty\right) \text { by an infinitesimal value and } \\
\text { contracts inward (compression) from all } \\
\text { sides approaching a negative unit radius } \\
\left({ }^{D} r \approx-1\right) \text { and then tends to a radius } \\
\text { tending to zero }\left({ }^{D} r \rightarrow 0\right) \text { to } C r \\
\text { As explained below (see section } 3.10) \text {, the } \\
\text { value of }{ }^{D} r=-1 \text { synchronous with value is } \\
{ }^{L} r=1 \text { in }{ }^{L} S T \text { is impossible. }\end{array}$ \\
\hline 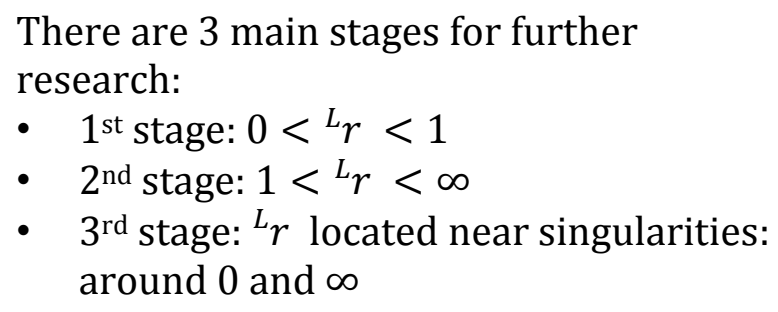 & 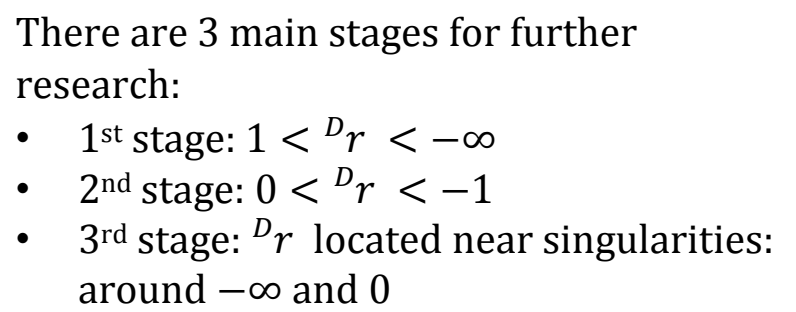 \\
\hline
\end{tabular}

To study the singularities indicated at the $3^{\text {rd }}$ stage in both space-times ${ }^{L} S T$ and ${ }^{D} S T$, one can use particular the infinitesimal (non-standard) analysis $[67,68,69]$. One of the criteria for the effectiveness of the infinitesimal analysis (as well as any other) in both space-times should be an explanation of the numerical value of the speed of light: $c$ in ${ }^{L} S T$ and, accordingly, the average speed of dark light: $-\frac{1}{c}$ in ${ }^{D} S T$. It is important to remember that, at any moment $T_{\text {synch }}$, the conditions of equations 19L and 19D are satisfied.

The equivalence of space-times ${ }^{L} S T$ and ${ }^{D} S T$, as well as the fact that they are antipodes to each other, lead to an amazing paradox of reference point priority (or view points). Considering the initial values of the parameters in equations 19L and 19D, divided into maximal and minimal, the derived results are presented in Table 3, considering the choice of priority of reference points and element signatures in ${ }^{L} S T$ and ${ }^{D} S T$. 
Chapter 3 Primary (maternal) Absolute space-time ${ }^{A} S T$ as the basis for the Big Bang model and subsequent Crunch

Table 3. The initial values of six pairs of basic parameters in equations 19L and 19D, divided into maximals and minimals, as well as choosing the reference point priority. These maximals and minimals are directly opposite to each other and depend on which space-time ${ }^{L} S T$ or ${ }^{D} S T$ is prioritized by the researcher. Such subjectivity leads to the paradox of the choice of a reference point priority (observation).

\begin{tabular}{|c|c|c|c|}
\hline \multicolumn{2}{|c|}{ Reference point priority in ${ }^{L} S T$} & \multicolumn{2}{c|}{ Reference point priority in ${ }^{D} S T$} \\
\hline \multicolumn{2}{|c|}{ Signature element $\left[+1^{+1}\right]$} & \multicolumn{2}{|c|}{ Signature element $\left[-1^{-1}\right]$} \\
\hline${ }^{L} K_{0}-\max$ & ${ }^{D} K_{0}-\min$ & ${ }^{L} K_{0}-\min$ & ${ }^{D} K_{0}-\max$ \\
\hline${ }^{L} \omega_{0}-\max$ & ${ }^{D} \omega_{0}-\min$ & ${ }^{L} \omega_{0}-\min$ & ${ }^{D} \omega_{0}-\max$ \\
\hline${ }^{L} S_{0}-\min$ & ${ }^{D} S_{0}-\max$ & ${ }^{L} S_{0}-\max$ & ${ }^{D} S_{0}-\min$ \\
\hline${ }^{L} t_{0}-\min$ & ${ }^{D} t_{0}-\max$ & ${ }^{L} t_{0}-\max$ & ${ }^{D} t_{0}-\min$ \\
\hline${ }^{L} v_{0}-\max$ & ${ }^{D} v_{0}-\min$ & ${ }^{L} v_{0}-\min$ & ${ }^{D} v_{0}-\max$ \\
\hline${ }^{L} G r_{0}-\min$ & ${ }^{D} G r_{0}-\max$ & ${ }^{L} G r_{0}-\max$ & ${ }^{D} G r_{0}-\min$ \\
\hline
\end{tabular}

This paradox requires a separate special consideration. In this article, when confronted with it, it will be handled using the conditions of the specific task (for example, see section 6.7 of Chapter 6). However, it is important to note that if the initial primary Absolute space-time ${ }^{\mathrm{A}} \mathrm{ST}$ is the priority of the researcher, then this paradox is under control.

Conclusion: equation 15 , the signature $\left[\cdot 1^{2}, \cdot\left(+1^{+1}\right), \cdot\left(-1^{-1}\right), \leftrightarrow\right]$, and the attained equations 19L and 19D are promising and serve as basis, but they require further research and expansion. The meaning of these equations will be discussed throughout this article multiple times.

\subsection{Algorithms and partial bundle of Absolute space-time ${ }^{A} S T$}

The previous examples detailed cases of complete bundle of Absolute space-time ${ }^{A} S T$. However, there is another possibility of partial bundle of Absolute space-time ${ }^{A} S T$, which can, for example, significantly affect the ratio of densities of tissues of space-times (and, accordingly, the ratio of the densities of types of matter and energy) in the Universe.

To address this, we can use the signature $\left[\cdot 1^{4}, \cdot\left(+1^{+1}\right), \cdot\left(-1^{-1}\right), \leftrightarrow\right]$. The left and right side of equation 15 were raised to the power of 4 and are presented in the following form:

$$
K^{2} \cdot K \cdot(K) \cdot \Omega^{2} \cdot \Omega \cdot(\Omega) \cdot S^{2} \cdot S \cdot(S)=V^{2} \cdot V \cdot(V) \cdot T^{2} \cdot T \cdot(T) \cdot G_{r}^{2} \cdot G_{r} \cdot\left(G_{r}\right)
$$

Each factor in brackets of the left and right sides of equation 21 was multiplied by $\left(-1^{-1}\right)$ to get:

$$
K^{2} \cdot K \cdot\left(-\frac{1}{K}\right) \cdot \Omega^{2} \cdot \Omega \cdot\left(-\frac{1}{\Omega}\right) \cdot S^{2} \cdot S \cdot\left(-\frac{1}{S}\right)=V^{2} \cdot V \cdot\left(-\frac{1}{V}\right) \cdot T^{2} \cdot T \cdot\left(-\frac{1}{T}\right) \cdot G_{r}^{2} \cdot G_{r} \cdot\left(-\frac{1}{G_{r}}\right)
$$

Carrying out the operation of partial bundle with equation 22, to obtain: 
In Absolute space-time ${ }^{A} S T$ equation:

$$
K^{2} \cdot \Omega^{2} \cdot S^{2}=V^{2} \cdot T^{2} \cdot G_{r}^{2}
$$

in space-time ${ }^{L} S T$, equation 19L, and in space-time ${ }^{D} S T$ equation 19D.

Conclusion: with partial bundle of Absolute space-time ${ }^{A} S T$, the contribution of ${ }^{L} S T$ and ${ }^{D} S T$ to the total density of matter and energy in the Universe is significantly reduced, and, accordingly, an accurate definition of relations between the densities ${ }^{A} S T$, and $\left({ }^{L} S T\right.$ and $\left.{ }^{D} S T\right)$ together is required.

\subsection{Algorithms and the Controlling Superimaginary Unit ${ }_{S} I$}

The main question that must be answered in this section is how do the two mutually transparent non-commutative space-times ${ }^{L} S T$ and ${ }^{D} S T$ self-consistent? $B B$ happened not in one central point, but in an infinite number of points in the Universe. And, accordingly, in both space-times at the moment of $B B$, an infinite number of fundamentally entangled, point-centers in ${ }^{L} S T$ and infinities in ${ }^{D} S T$ were born, that interact with each other through the Paracomplex Absolute space-time ${ }_{I}^{A} S T$ and form dominant development areas (selfconsistent homogenous expansion ${ }^{L} S T$ and heterogeneous contraction ${ }^{D} S T$ ). What is this generalized Paracomplex Absolute space-time ${ }_{I}^{A} S T$ ? Is it exclusively primary (maternal) Absolute space-time ${ }^{A} S T$ ? Or, is it a Controlling imaginary substance inextricably linked to ${ }^{A} S T$ in the period from $B B$ to $C r$ and forming together with it generalized Paracomplex ${ }_{I}^{A} S T$ ?

To answer these important questions, it is vital to note that the appearance of a square root in equation 1 , that defines the metric in ${ }^{A} S T$, is not accidental. Already at this very first stage, there is a hint to the complex nature of the numbers in ${ }^{L} S T$ and the dark complex nature of the numbers in ${ }^{D} S T$ as well as Paracomplex nature of primary numbers in ${ }^{A} S T$. Evidently, the self-consistency of interactions between space-times ${ }^{L} S T$ and ${ }^{D} S T$, the use of complex numbers in ${ }^{L} S T$ and dark complex numbers in ${ }^{D} S T$ is unavoidable. Section 3.5 briefly states the argument in favor of introducing a dark imaginary hyperbolic unit $\tilde{\imath}$ in an algebra with a dominant minus. It also offers an extended definition of TFCV. This theory is also a local model-bridge, combining the properties of the traditional algebra with an absolute dominant plus (used in ${ }^{L} S T$ ) and algebra with a dominant minus. At the same time, the introduction of the concept of a dark imaginary hyperbolic unit in an algebra with an absolute dominant minus allows to create a theoretical model-bridge based on dark complex numbers, combining the properties of "dark" algebra and algebra with an absolute dominant plus. If the applied signature $(-1)$ allowed to suggest a hypothesis of some properties of a set of dark numbers $(\mathbb{D})$, then the applied signature $\left(-1^{-1}\right)$ offers information in regards to their geometric arrangement relative to a set of real numbers $(\mathbb{R})$. Considering the properties of a well-known function $y=\frac{1}{x}$ (the inversion of the action of values of dark numbers from the abscissa axis $x$ to the ordinate axis $y$ is significant), leads to the conclusion that dark numbers $D a$ act as if they are arranged mutually perpendicular relatively to axis of real numbers $R e$. It can also be assumed that both theories, TFCV and the future theory based on a dark imaginary hyperbolic unit $\tilde{l}$, agree with two complex planes (Figure 5), which should 
unite on a generalized Supercomplex plane (Figure 6) and ensure self-consistency in the interactions of tissues of space-times ${ }^{L} S T$ and ${ }^{D} S T$.

Space-time ${ }^{L} S T$

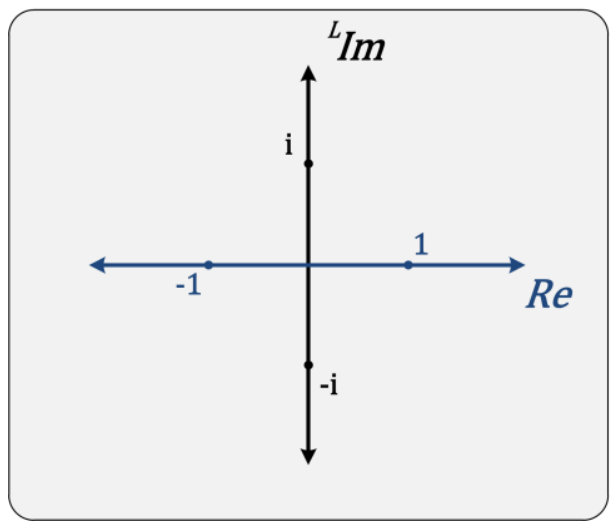

Space-time ${ }^{D} S T$

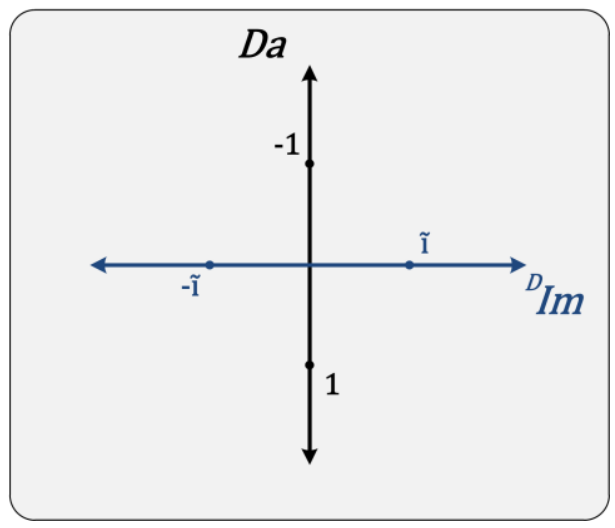

Figure 5. Complex plane in ${ }^{L} S T$ and hypothetical dark complex plane in ${ }^{D} S T$. On the left is a customary mathematical in ${ }^{L} S T$, with imaginary elliptical unit $i$, where $R e$ is the axis of real numbers, and ${ }^{L} I m$ is the axis of imaginary numbers. On the right is the possible dark complex plane in ${ }^{D} S T$, with imaginary hyperbolic unit $\tilde{l}$ which, based on the proposed hypothesis, is formed in a very non-trivial way (inversions actions of the values of dark numbers from abscissa axis $x$ to the ordinate axis $y$ ), where $D a$ is the action projections axis of dark numbers, and ${ }^{D} I m$ is the axis of dark imaginary numbers.

Another argument in favor of this theory is that the speed of light $c$ in ${ }^{L} S T$ for an infinite Universe is an insignificant value. The concept of the average speed of dark light: $-\frac{1}{c}$ (for an algebra with an absolute dominant minus) and its properties in ${ }^{D} S T$ still remain to be studied, however, these properties are unlikely to change the way this problem is addressed). Given these factors, the instantaneous imaginary time it in ${ }^{L} S T$ and instantaneous dark imaginary time $\tilde{i} t$ in ${ }^{D} S T$ in the formulas, will inevitably ensure selfconsistency (in $T_{\text {synch }}$ ) of the interactions of tissues of space-time ${ }^{L} S T$ and ${ }^{D} S T$ in the entirety (in any areas) of the infinite Universe.

This idea can be further developed based on well-known studies of the generalization of complex numbers in geometry, curvature of space and universal algebra [70, 71, 72]. Introducing a Controlling Superimaginary unit, designated as ${ }_{S} I$, with square value which is depending on the curvature of space-time:

$$
S^{2}=\left\{\begin{array}{cc}
\text { for } \Omega=0 \text { in }{ }^{A} S T: I^{2}=\infty \text { and } I^{2}=0 \text { (where, } I-\text { parabolic unit) } \\
\text { for } \omega>0 \text { in }{ }^{L} S T: & i^{2}=-1 \text { (where, } i \text { - elliptical unit) } \\
\text { for } \omega<0 \text { in }{ }^{D} S T: & \tilde{\imath}^{2}=+1 \text { (where, } \tilde{\imath} \text { - hyperbolic unit) }
\end{array}\right.
$$

It must be emphasized that in contrast to the studies presented in Lavrentiev M. A. and Shabat B. V. [73] where generalized complex numbers are introduced in one single spacetime (evidently this is ${ }^{L} S T$ ), in proposed case, the question regarding introduction of zero divisor into algebra with imaginary hyperbolic unit $\tilde{l}$ applied in ${ }^{D} S T$, requires a separate 
special consideration (see column 3 of Table 4). Moreover, for reasons of accuracy (or removing possible contradictions), a hypothesis must be introduced that a space-time ${ }^{D} S T$ that uses dark numbers, dark complex numbers, dark quaternions, etc., a theorem, equivalent to Frobenius's theorem [74], operating in space-time ${ }^{L} S T$, must operate.

Eventually, by operating three space-times, a new way of defining generalized complex numbers is examined, in which the square of the Controlling Superimaginary Unit ${ }_{S} I^{2}$ depends on the curvature of that space-time. In the moment $B B$, in primary (maternal) Absolute space-time ${ }^{A} S T$ of zero $(\Omega=0)$ curvature (this equality, as well as the first line of equation 24.0 will be supplemented and clarified in section 4.2 of Chapter 4), an imaginary parabolic unit $I$ is realized, the square of which can simultaneously take two values $\left(I^{2}=\infty\right.$ and $\left.I^{2}=0\right)$. In space-time of positive curvature ${ }^{L} S T$, an imaginary elliptic unit $i\left(i^{2}=-1\right)$ is realized. In space-time of negative curvature ${ }^{D} S T$, an imaginary hyperbolic unit $\tilde{l}\left(\tilde{l}^{2}=+1\right)$ is realized.

\subsection{Parabolic imaginary unit $I$}

To understand the role of the imaginary parabolic unit $I$ in space-time ${ }^{A} S T$, a hypothesis must be suggested, that in the primary (maternal) Absolute space-time ${ }^{A} S T$, at the moment of $B B$ the imaginary parabolic unit $I$ has following properties: its square can give two values simultaneously: $I^{2}=\infty$ and $I^{2}=0$;

Stemming from this hypothesis is the validity of the following relations in ${ }^{A} S T$ :

$$
0 \times \infty=1 ; 1 / 0=\infty ; 1 / \infty=0 ;
$$

Perhaps, two relations should be added to them:

$$
0 / \infty=\mathbf{1} ; \infty / 0=\mathbf{1}
$$

Based on the above, the properties of an imaginary parabolic unit $I$ allows to ultimately eliminate all possible uncertainties associated with these concepts and/or numerical values that act only in Absolute space-time ${ }^{A} S T$ and do not apply to space-times ${ }^{L} S T$ and ${ }^{D} S T$.

The main goal and task of the imaginary parabolic unit $I$ is to use its properties to initiate $B B$, by conducting several synchronous processes:

- Reduction of all basic parameters to values equal to the imaginary parabolic unit 1

- Initiating the parabolic bundle of all the basic parameters that triple, and additionally form pairs: 0 and $+\infty$ or 0 and $-\infty$, and accordingly

- Absolute space-time ${ }^{A} S T$ triples

- These born space-times are mutually transparent and are subdivided by sign: spacetime of positive curvature ${ }^{L} S T$ and space-time of negative curvature ${ }^{D} S T$

- In the space-time of positive curvature ${ }^{L} S T$ all $+\infty$ and 0 are involved, if the partner parameter 0 has the value $-\infty$ (Table 3 )

- In the space-time of negative curvature ${ }^{D} S T$, all $-\infty$ and 0 are involved, if the partner parameter 0 has a value of $+\infty$ (Table 3 ) 
- Distribution of pairs of initial value basic parameters in space-times ${ }^{L} S T$ and ${ }^{D} S T$ takes place in such a way that interspatial potential ${ }^{S T} U$ arises in generalized Paracomplex ${ }_{I}^{A} S T$ (Table 4)

Table 4. The initial values of basic parameters at the moment of $B B$ in three space-times ${ }_{I}^{A} S T,{ }^{L} S T$ and ${ }^{D} S T$. Data in the columns 2 and 3 is shown in accordance with the choice of priority of reference points and signature elements in ${ }^{L} S T$ and ${ }^{D} S T$ (see section 3.7 of the current Chapter).

\begin{tabular}{|c|c|c|c|c|}
\hline \multirow{3}{*}{${ }^{A} S=\mathbf{1}(\text { constant })^{*}$} & \multirow{2}{*}{\multicolumn{2}{|c|}{$\begin{array}{c}\text { Space-time }{ }^{L} S T \\
\text { Reference point priority } \\
\text { with signature element } \\
\qquad\left[+1^{+1}\right]\end{array}$}} & \multicolumn{2}{|l|}{ Space } \\
\hline & & & \multicolumn{2}{|c|}{$\begin{array}{c}\text { Reference point priority } \\
\text { with signature element } \\
\qquad\left[-1^{-1}\right]^{* *}\end{array}$} \\
\hline & ${ }^{L} S_{0}=0$ & ${ }^{D} S_{0}=-\infty$ & ${ }^{L} S_{0}=\frac{1}{0}$ & ${ }^{D} S_{0}=-\frac{1}{\infty}$ \\
\hline${ }^{A} \Omega=\mathbf{1}(\text { constant })^{*}$ & ${ }^{L} \omega_{0}=\infty$ & ${ }^{D} \omega_{0}=0$ & ${ }^{L} \omega_{0}=\frac{1}{\infty}$ & ${ }^{D} \omega_{0}=\frac{1}{0}$ \\
\hline${ }^{A} T=\mathbf{1}(\text { constant })^{*}$ & ${ }^{L} t_{0}=0$ & ${ }^{D} t_{0}=-\infty$ & ${ }^{L} t_{0}=\frac{1}{0}$ & ${ }^{D} t_{0}=-\frac{1}{\infty}$ \\
\hline${ }^{A} V=\mathbf{1}(\text { constant })^{*}$ & ${ }^{L} v_{0}=\infty$ & ${ }^{D} v_{0}=0$ & ${ }^{L} v_{0}=\frac{1}{\infty}$ & ${ }^{D} v_{0}=\frac{1}{0}$ \\
\hline${ }^{A} G r=\mathbf{1}(\text { constant })^{*}$ & ${ }^{L} G r_{0}=0$ & ${ }^{D} G r_{0}=-\infty$ & ${ }^{L} G r_{0}=\frac{1}{0}$ & ${ }^{D} G r_{0}=-\frac{1}{\infty}$ \\
\hline${ }^{A} K=\mathbf{1}(\text { constant })^{*}$ & ${ }^{L} K_{0}=\infty$ & ${ }^{D} K_{0}=0$ & ${ }^{L} K_{0}=\frac{1}{\infty}$ & ${ }^{D} K_{0}=\frac{1}{0}$ \\
\hline \multicolumn{5}{|c|}{$\begin{array}{l}\text { * The values should be expressed by Paracomplex primary numbers, which the } \\
\text { theory is currently lacking } \\
\text { ** For algebra with absolute dominant minus with signature element }\left[-1^{-1}\right] \text { requires } \\
\text { introduction of zero divisor }\end{array}$} \\
\hline
\end{tabular}

- Imaginary parabolic unit $I$ also triples, giving rise to an imaginary elliptical unit $i$, whose square: $i^{2}=-1$, acts in space-time of positive curvature ${ }^{L} S T$, and an imaginary hyperbolic unit $\tilde{\imath}$, whose square: $\tilde{l}^{2}=+1$, acts in space-time of negative curvature ${ }^{D} S T$

As previously suggested, three imaginary units form a generalized Paracomplex plane (Figure 6), combining the complex plane for ${ }^{L} S T$ and the dark complex plane for ${ }^{D} S T$, which plays, according to this hypothesis, the main controlling role in the coordination of all interactions in space-time ${ }^{L} S T$ and ${ }^{D} S T$, as a result creates opportunity of "selfconsistency effect" to be realized in the Universe. 


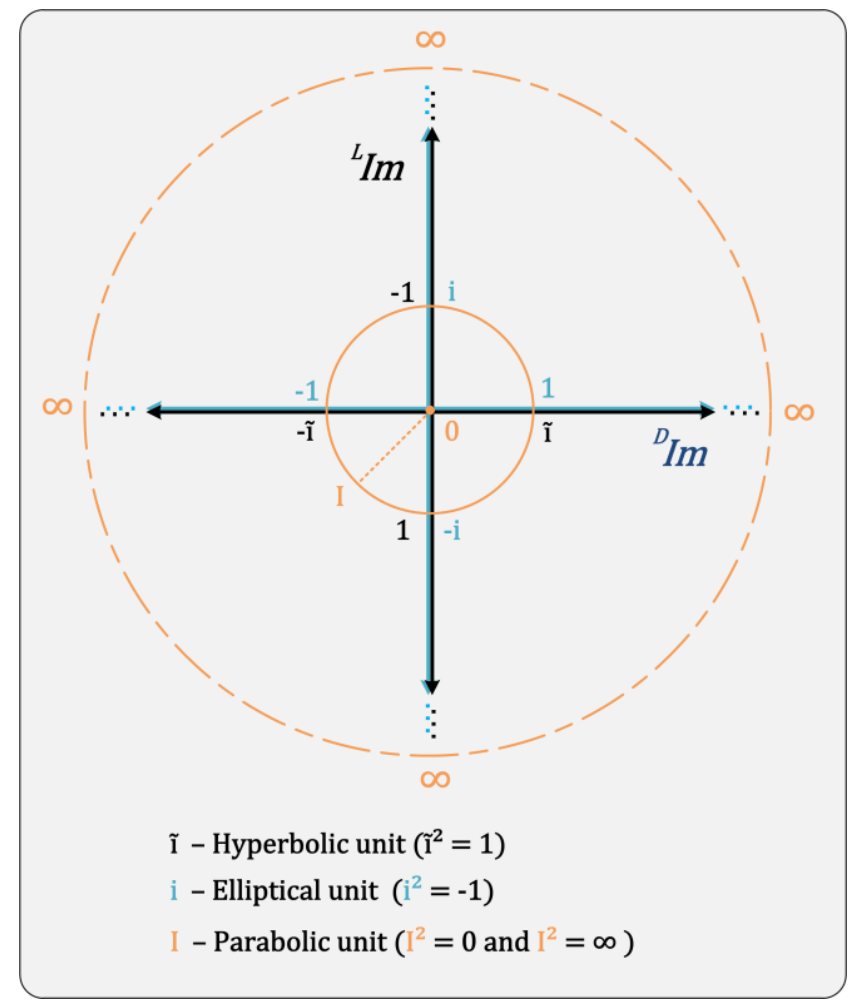

Figure 6. Generalized Paracomplex plane. Combining a complex plane with the imaginary elliptic unit $i$ in ${ }^{L} S T$, dark complex plane with imaginary hyperbolic unit $\tilde{l}$ in ${ }^{\mathrm{D}} S T$, as well as the value of imaginary parabolic unit $I$ in ${ }^{\mathrm{A}} S T$.

Conclusion: it is necessary to find an algorithm and a signature that include the Controlling Superimaginary Unit ${ }_{s} I$ in order to derive formulas that expand to complexification equations 15 or 23 with the participation of an imaginary parabolic unit $I$, and, accordingly, the subsequent bundle of these equations to 19L, with participation of an elliptical imaginary unit $i$, and 19D, including the hyperbolic imaginary unit $\tilde{l}$, up to 4 spacial and 2 temporal dimensions (for each space-time). This approach should provide a solution to the tasks outlined in this chapter.

\subsection{About dark numbers}

Two questions inevitably arise from the results obtained in sections 3.5, 3.7, 3.9 of this chapter. First, what are dark numbers $D a$ and the set of dark numbers $(\mathbb{D})$ ? Second, are dark numbers an exact copy of real numbers from ${ }^{L} S T$, different only in sign, and acting as if they are located mutually perpendicular with respect to them? In order to try to answer these questions it is necessary to consider the following factors:

Taking into account the initial provisions of Chapter 2, the Supercomplex generalized plane obtained (Figure 6), and the very essence of a stationary self-consistent Universe, it becomes 
Chapter 3 Primary (maternal) Absolute space-time ${ }^{A} S T$ as the basis for the Big Bang model and subsequent Crunch

obvious that the Universe is mathematical in nature [75]. This leads to the important conclusion that the concept of number is not an abstract element, but the most important mathematical tool necessary to fulfill the tasks and functions assigned to it. Thus, the set of real numbers $(\mathbb{R})$ must strictly correspond to the properties of space-time ${ }^{L} S T$. These (real numbers) should be programmed in the algorithm for the birth and development of this space-time and allowed to be described from the moment of $B B$ to $\mathrm{Cr}$. For example, spacetime ${ }^{L} S T$ is homogeneous and isotropic (on large scales), expanding from the moment of $B B$ to $\mathrm{Cr}$. The distance in it is continuous, which corresponds to the axiom of completeness of the set of real numbers. Time ${ }^{L} T$ is homogeneous and isotropic. In space-time ${ }^{L} S T$ the Archimedean metric is realized.

Similarly, the set of dark numbers $(\mathbb{D})$ should strictly correspond to the properties of spacetime ${ }^{D} S T$ and allowed to be described from the moment of $B B$ to $C r$. For example, space-time ${ }^{D} S T$ is heterogeneous and anisotropic and contracts from the moment of $B B$ to $\mathrm{Cr}$. Time ${ }^{D} T$ is heterogeneous and anisotropic. The definition of distance in ${ }^{D} S T$, that can be discontinuous, is the subject of further research. In space-time ${ }^{D} S T$, a non-Archimedean metric is realized.

In accordance with the proposed theory, both space-times ${ }^{L} S T$ and ${ }^{D} S T$ are forced to interact with each other through generalized Paracomplex Absolute space-time (see Chapters 6-11). In accordance with Ostrowski's theorem, the field of rational numbers $Q$ can be replenished in two equivalent ways: either to the field of real numbers $\mathbb{R}$, or to the field of $\mathrm{p}$-adic numbers $Q_{p}$.

It is obvious that the field of real numbers $\mathbb{R}$ is applied in space-time ${ }^{L} S T$, corresponding to its properties. And, as for the p-adic numbers, in accordance with the proposed hypothesis, it finds its application in space-time ${ }^{D} S T$ to a much greater extent. The main reason for this statement is that the properties of p-adic numbers are more consistent with nonArchimedean space-time ${ }^{D} S T$ properties (see Chapters 5-11). The assumption is that on the Planck scales $\left(\sim 10^{-34} \mathrm{~cm}\right)$, the properties of space-time ${ }^{L} S T$ can change dramatically, and here is where p-adic quantum physics finds its application, does not have sufficient grounds. However, the discussions about the "object" of the applicability of p-adic numbers and their classification have practically no effect on the value of studies already carried out in this field, and in no way diminish the enormous contribution made to the development of p-adic physics over the years $[76,77]$.

Thus, the answer to the second question will be an unequivocal "no". As for the first question, dark numbers $D a$ (set of dark numbers $\mathbb{D}$ ) require further in-depth study, as do the properties of space-time ${ }^{D} S T$, in which they are realized and must be described in.

\subsection{Summary of the preliminary results of Chapter 3}

As a result of this theoretical research, at the moment of $B B$ primary (maternal) Absolute space-time ${ }^{A} S T$ with Euclidean geometry $(\varepsilon)$ with a reference metric, with static tissue of 
space and Controlling Superimaginary Unit ${ }_{S} I$ form a generalized Paracomplex Absolute space-time ${ }_{I}^{A} S T$ including sphere with basic parameters which are equal to imaginary parabolic unit $I\left(S_{s p h}=I, \Omega_{s p h}=I,{ }^{A} T=I,{ }^{A} V=I,{ }^{A} G r=I,{ }^{A} K=I\right)$.

At the same time, as a result of the partial parabolic bundle ${ }^{A} S T$, two mutually transparent non-commutative self-consistent space-times were born and dynamically developing in it: ${ }^{L} S T$ and ${ }^{D} S T$ (Figures 1 and 4 ).

Based on the fact that the fate of those formed in $T_{\text {synch }}$ in generalized Paracomplex Absolute space-time ${ }_{I}^{A} S T$ at the moment $B B$ of two space-times ${ }^{L} S T$ and ${ }^{D} S T$ is predetermined: in the future, they will disappear at the moment $C r$, also in $T_{\text {synch }}$, the self-consistent model $B B-$ $\mathrm{Cr}$ is logically completed.

Evidently, the above algorithm examples of the complete and partial parabolic bundle of the Absolute space-time ${ }^{A} S T$ are preliminary and incomplete. They require further comprehension, reflection and research; however, the one correct algorithm remains to be found.

Conclusion: the main tasks of TSSCU are to determine the exact definition of the main parameters in the model $B B-C r$ (paired and self-consistent), the initial conditions for their interactions, the correct bundle algorithm ${ }^{A} S T$ and self-consistent formulas, and the emergence and further development of space-times ${ }^{L} S T$ and ${ }^{D} S T$.

To achieve this goal, the properties and characteristics of each of the three space-times: ${ }^{A} S T$, ${ }^{L} S T$ and ${ }^{D} S T$ need to be considered. 


\section{Chapter 4 Properties and characteristics of the primary (maternal) Absolute space-time ${ }^{A} S T$}

\subsection{Introduction}

It should be noted that there are objective and other various difficulties in order to explore and to describe properties ${ }^{A} S T$. Investigation of the main mathematical object introduced in this work, Controlling Superimaginary Unit ${ }_{S} I$ is at its initial stage. It is unknown, whether the relationship between ${ }_{S} I$ and ${ }^{A} S T$ is both independent and mutually transparent, as is the relationship of idealistic and primary material origin? It is also unknown, what makes ${ }_{s} I$ initiate the process $B B$ and subsequent $C r$ in ${ }^{A} S T$ ? And finally, it is unknown what kind of mathematical objects are: primary numbers, which we use to describe properties in ${ }^{A} S T$; as well as Paracomplex numbers, which include an imaginary parabolic unit? However, some tentative assumptions and conclusions, at the hypothesis and intuition level, can be discussed in this Chapter to stimulate discussion on these critical topics.

\subsection{Conformity of each 0 and $\infty$. The inseparability of these concepts and meanings from each other in space-time ${ }^{A} S T$}

A natural question arises, what can be equal to the basic parameters in the primary (maternal) ${ }^{A} S T$ ? Considering that fact that in ${ }^{A} S T$, Euclidean geometry with zero ${ }^{A} \Omega=0$ curvature is realized, it can be assumed that they are equal to the following values:

${ }^{A} S^{2}=\infty ;{ }^{A} T^{2}=\infty ;{ }^{A} \Omega=0 ;{ }^{A} V=0 ;{ }^{A} G r^{2}=\infty ;{ }^{A} K=0$.

But then, a second question arises about the correctness of such values in the primary (maternal) Absolute space-time ${ }^{A} S T$ ? Understanding this issue using the example of the distance-radius of a sphere, the value of which is assumed to be equal to ${ }^{A} S^{2}=\infty$. Per author's hypothesis, the impossibility of determining 0 , makes the assumed value $\infty$ incorrect. This is since a pair of values: $\infty$ and 0 are inseparable from each other in ${ }^{A} S T$. For each 0 there must be strictly its own, corresponding to it and only $\infty$. And vice versa, each $\infty$ there must be strictly its own, corresponding to it and only 0 . This hypothesis about the correspondence and continuity of each zero 0 and its infinity $\infty$, and vice versa leads to the fact all six basic parameters in ${ }^{A} S T$ have two values simultaneously. Namely:

- $\quad$ distance-radius of sphere: ${ }^{A} S^{2}=0$ and ${ }^{A} S^{2}=\infty$;

- time: ${ }^{A} T^{2}=0$ and ${ }^{A} T^{2}=\infty$;

- $\quad$ curvature: ${ }^{A} \Omega=0$ and ${ }^{A} \Omega=\infty$;

- $\quad$ velocity: ${ }^{A} V=0$ and ${ }^{A} V=\infty$;

- $\quad$ gravity: ${ }^{A} G r^{2}=0$ and ${ }^{A} G r^{2}=\infty$;

- temperature: ${ }^{A} K=0$ and ${ }^{A} K=\infty$.

This approach seems unexpected only at the first glance. After deeper consideration, it becomes natural and logical. In accordance with it, Euclidean geometry realized in ${ }^{A} S T$ must be in consensus with both values of curvature ${ }^{A} \Omega=0$ и ${ }^{A} \Omega=\infty$, which act simultaneously. 
And, equation 24.0, which defines generalized complex numbers, must be corrected and written in the following way:

$$
{ }_{S} I^{2}=\left\{\begin{array}{ccc}
\text { for }{ }^{A} \Omega=0 \text { and }{ }^{A} \Omega=\infty \text { in }{ }^{A} S T: I^{2}=\infty \text { and } I^{2}=0 \text { (where, } I \text { - parabolic unit) } \\
\text { for } \omega>0 \text { in }{ }^{L} S T: & i^{2}=-1 & \text { (where, } i \text { - elliptical unit) } \\
\text { for } \omega<0 \text { in }{ }^{D} S T: & \tilde{l}^{2}=+1 & \text { (where, } \tilde{\imath} \text { - hyperbolic unit) }
\end{array}\right.
$$

Thus, this hypothesis provides new opportunities for understanding and developing the $B B$ - $\mathrm{Cr}$ model.

\subsection{Changing the properties and characteristics of space-time ${ }^{A} S T$ depending on the period of Relaxation or from period of Big Bang to subsequent Crunch}

In accordance with provisions, set forth in the previous Chapters 2 and 3, the properties and characteristics of the primary (maternal) Absolute space-time ${ }^{A} S T$ radically change depending on two successive periods (see Figure 7). The first period begins when the Controlling Superimaginary Unit ${ }_{S} I$ interacts with ${ }^{A} S T$ and the next Big Bang $\left(B B_{n}\right)$ arises with subsequent Crunch $\left(\mathrm{Cr}_{n}\right)$ and so on. The second period of Relaxation (or $C r_{n}-B B_{n+1}$ and so on) occurs at the moment of the next $\mathrm{Cr}$, when Controlling Super-Imaginary Unit $s^{I}$ stops interacting with ${ }^{A} S T$.

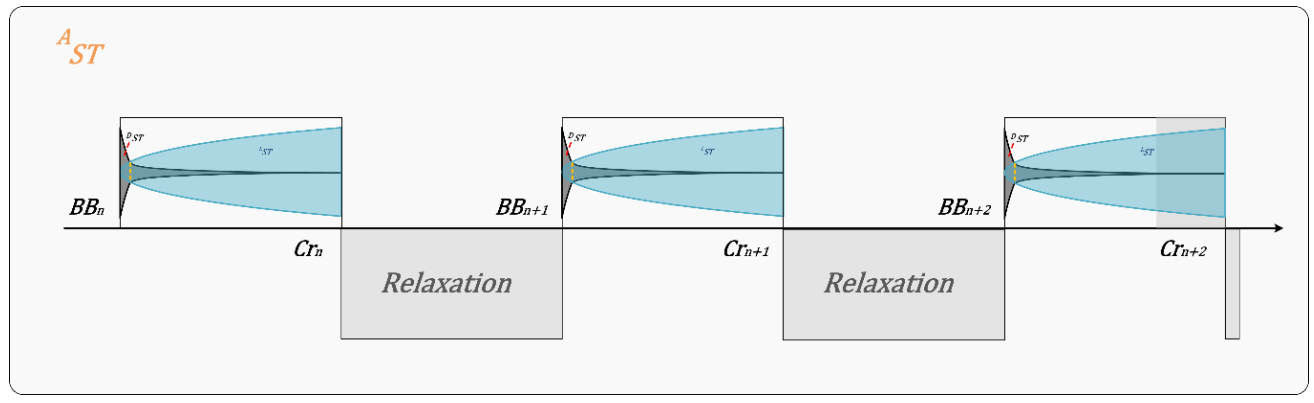

Figure 7. Periodogram $\boldsymbol{B} \boldsymbol{B}-\boldsymbol{C r}$ in the Universe. Primary (maternal) Absolute space-time ${ }^{A} S T$ with simultaneous zero $(\Omega=0)$ and infinity $(\Omega=\infty)$ curvatures serves as the basis for the oscillation of the Controlling Superimaginary Unit ${ }_{S} I$, which periodically initiates the Big Bang $B B$ with subsequent Crunch $\mathrm{Cr}$ and period of relaxation.

\subsection{Relaxation period $\left(\mathrm{Cr}_{\mathrm{n}-1}-\mathrm{B} B_{\mathrm{n}}\right)$. The state of uncertainty of the basic parameters}

At the moment $\mathrm{Cr}$, Controlling Superimaginary Unit ${ }_{S} I$ stops interacting with ${ }^{A} S T$. Accordingly, the primary (maternal) Absolute space-time ${ }^{A} S T$ becomes empty, lifeless, devoid of any processes and units of measurement, due to the absence of an imaginary parabolic unit $I$, that sets relations 25 and 26.

Based on the foregoing, it can be assumed that all basic parameters are in a state of uncertainty during the relaxation period.

Conclusion: the primary (maternal) Absolute space-time ${ }^{A} S T$ in the relaxation period or $C r_{n-1}-B B_{n}$ is in the state of uncertainty. 


\subsection{Period from Big Bang to subsequent Crunch $\left(B B_{n}-C r_{n}\right)$}

Based on the conclusion of the previous paragraph, the role of the Controlling Superimaginary Unit ${ }_{S} I$, that initiates $B B$ becomes more meaningful. It introduces into the primary (maternal) Absolute space-time ${ }^{A} S T$ the much-needed imaginary parabolic unit, establishing the relations 25 and 26.

Considering the processes of the imaginary parabolic unit $I$ in the moment (in anticipation) of $B B$ on example equation 1 , where $S$ is the distance-radius of sphere equal to:

$$
S^{2}=\left(d x^{2}+d y^{2}+d z^{2}\right)
$$

The right side of this equation, according to the assumption in ${ }^{A} S T$, can be equal to the minimum and the maximum correct value in mathematics: 0 and $+\infty$. Then, accordingly, if $S^{2}=0$ :

$$
S=\sqrt{0}
$$

At the moment of $B B$, instead of 0 , the square of the imaginary parabolic unit $I^{2}$ takes effect, resulting in:

$$
S=\sqrt{I^{2}}
$$

Further, accordingly:

$$
S=I=\mathbf{1}
$$

If $S^{2}=\infty$ :

$$
S=\sqrt{\infty}
$$

At the moment of $B B$, instead of $\infty$, the square of the imaginary parabolic unit $I^{2}$ takes effect, resulting in:

$$
S=\sqrt{I^{2}}
$$

Further, accordingly:

$$
S=I=1
$$

In such amazing ways, that need meticulous criticism, results in a Controlling sphere with a distance-radius equal to imaginary parabolic unit, which, as in the domino effect, completely transforms equation 15 , which sets the start to parabolic bundle in ${ }^{A} S T$ and the beginning of another period $B B-C r$. 
Below are some of the properties and characteristics of ${ }_{I}^{A} S T$.

1. Dimension

At the moment of $B B$ and till $\mathrm{Cr}$ the primary (maternal) Absolute space-time ${ }^{A} S T$ and imaginary parabolic unit $I$ form a generalized Paracomplex space-time ${ }_{I}^{A} S T$, consisting of 6 paracomplex dimensions:

- 3 spatial coordinates ${ }^{A} S T$

- 1 time ${ }^{A} T$ in ${ }^{A} S T$

- 1 imaginary parabolic spatial unit $I$

- 1 imaginary parabolic time ${ }_{I}^{A} T$

Consideration of the dimensions of self-consistent space-times ${ }^{L} S T$ and ${ }^{D} S T$ that are included in ${ }_{I}^{A} S T$ are not part of the purpose of this Chapter. They will be discussed in Chapter 5 and 12 of this article.

2. Value of basic parameters in ${ }_{I}^{A} S T$ :

- distance-radius of the sphere: ${ }^{A} S^{2}=0$ and ${ }^{A} S^{2}=\infty$ with imaginary parabolic unit $I$

- time: ${ }^{A} T^{2}=0$ and ${ }^{A} T^{2}=\infty$ with imaginary parabolic unit $I$

- curvature: ${ }^{A} \Omega=0$ and ${ }^{A} \Omega=\infty$ with imaginary parabolic unit $I$

- velocity: ${ }^{A} V=0$ and ${ }^{A} V=\infty$ with imaginary parabolic unit $I$

- gravity: ${ }^{A} G r^{2}=0$ and ${ }^{A} G r^{2}=\infty$ with imaginary parabolic unit $I$

- temperature: ${ }^{A} K=0$ and ${ }^{A} K=\infty$ with imaginary parabolic unit $I$

3. Basic categories in ${ }_{I}^{A} S T$ :

- Matter ${ }^{A} M$ is primary controlling with imaginary parabolic unit $I$

- Energy ${ }^{A} E$ is primary controlling with imaginary parabolic unit $I$

- Information ${ }^{A} I$ is primary controlling with imaginary parabolic unit $I$

4. Geometry in ${ }_{I}^{A} S T$ :

- Euclid + imaginary parabolic sphere with basic parameters equal to $I$

- Fifth postulate: in a plane through a point that does not lie on a given line, one can draw one and only one line parallel to this

- The sum of angles of a triangle is: $\angle \alpha+\angle \beta+\angle \gamma=180^{\circ}$

5. Entropy

Finding approaches to study the most important concept of entropy ${ }^{A} H$ in ${ }^{A} S T$ in both periods: : from $C r_{n-1}$ to $B B_{n}$ and from $B B_{n}$ to $C r_{n}$ is extremely promising and can provide answers to some questions.

Conclusions: many of the provisions, presented in this Chapter are debatable and require further comprehensive research. 


\section{Chapter 5 Comparative properties and characteristics of space-times ${ }^{L} S T$ and ${ }^{D} S T$}

Descriptions and comparisons of antagonistic properties and characteristics of two spacetimes ${ }^{L} S T$ and ${ }^{D} S T$ are given below.

\subsection{Dimension of space and time}

\begin{tabular}{|c|c|}
\hline Space-time ${ }^{L} S T$ & Space-time ${ }^{D} S T$ \\
\hline $\begin{array}{l}\text { - Space is 4-dimensional, with } 3 \text { spatial } \\
\text { coordinates and } 1 \text { dimension - time }{ }^{L} T \\
\text { - Time }{ }^{L} T \text { is objective, continuous, and } \\
\text { homogeneous } \\
\text { The direction (vector or arrow) of time } \\
\text { is from the past to the future } \\
\text { - Imaginary dimension: } 2 \text {-dimensional, } 1 \\
\text { imaginary elliptical unit } i \text { and } 1 \\
\text { dimension of imaginary elliptical time } \\
{ }_{i} t \text {. }\end{array}$ & 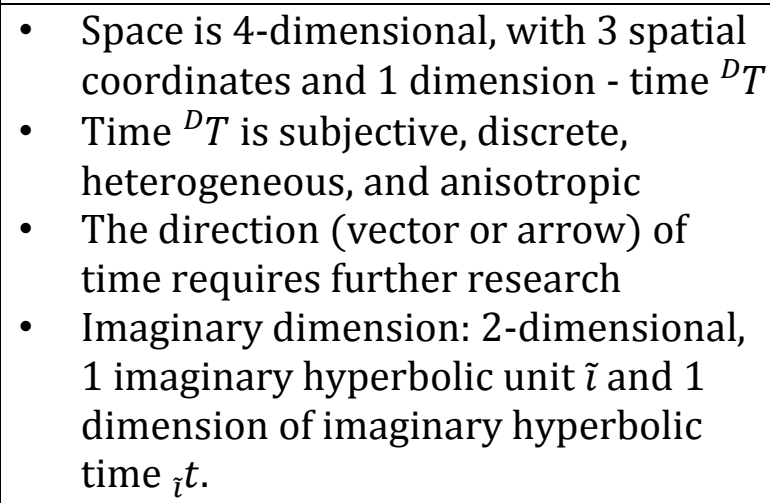 \\
\hline
\end{tabular}

5.2 General conformities

\begin{tabular}{|l|l|}
\hline \multicolumn{1}{|c|}{ Space-time ${ }^{L} S T$} & \multicolumn{1}{c|}{ Space-time ${ }^{D} S T$} \\
\hline $\begin{array}{l}\text { Space is homogeneous and isotropic; } \\
\text { the direct consequence of this }\end{array}$ & Space is heterogeneous and \\
statement are the exact fundamental & anisotropic. \\
constants in ${ }^{L} S T$ & It has a fractional dimension, fractal \\
& nature [78, 79, 80, 81, 82, 83], and, \\
& possibly, p-Adic structure [84, 85]. \\
& The direct consequence of these \\
& characteristics are the fluctuating \\
& parameters, tending in ${ }^{D} S T$ to the limit \\
& to values that are equivalent of exact \\
& constants in ${ }^{L} S T$ \\
\hline
\end{tabular}




\subsection{Properties and characteristics of time ${ }^{L} T$ and time ${ }^{D} T$}

\begin{tabular}{|c|c|}
\hline Space-time ${ }^{L} S T$ & Space-time ${ }^{D} S T$ \\
\hline $\begin{array}{l}\text { - } \text { Time of existence, }{ }^{L} T \text { starts at the } \\
\text { moment } B B \text { and ends at the moment } C r \\
\text { - Unit of time }{ }^{L} T \text { is conditional, referred } \\
\text { to as second } \\
\text { Time }{ }^{L} T \cong 14 \text { billion years (according } \\
\text { to modern data) } \\
\text { The ongoing temporary processes on } \\
\text { Earth have a certain cyclical nature } \\
\text { associated with the period of Earth's } \\
\text { revolution around the Sun: } \\
{ }^{L} T_{\text {period }}=365,25636604 \text { days }\end{array}$ & $\begin{array}{l}\text { - Time of existence, }{ }^{D} T \text { starts at the } \\
\text { moment } B B \text { and ends at the moment } \\
\text { - Un } \\
\text { - Time of time }{ }^{D} T \text { is not defined } \\
\text { - The ongoing temporary processes on } \\
\text { Earth have a certain cyclical nature } \\
\text { associated with the period of Moon's } \\
\text { revolution around the Earth [86]; in } \\
\text { this case, the duration of the main } \\
\text { measurement cycle in units } \\
{ }^{L} S T \text { is equal to the synodic lunar year: } \\
{ }^{D} T_{\text {period }}=29,530588 \text { days } \\
\text { Time }{ }^{D} T \text { can be described as internal, } \\
\text { hidden, local, p-Adic and wave-like } \\
\text { One of the most important properties } \\
\text { of time }{ }^{D} T \text { is its capability to fluctuate } \\
\text { and accelerate, changing its tempo } \\
\text { with positive and negative acceleration } \\
\text { (see section } 6.8 \text { ) } \\
\text { Despite the need to further understand } \\
\text { and research the properties of time } \\
{ }^{D} T \text {, there are works on mathematical } \\
\text { modeling of pseudo-time properties } \\
\text { [87, 88, 89], which in practice, allow to } \\
\text { obtain effective results. }\end{array}$ \\
\hline
\end{tabular}

5.4 Curvature, sum of triangle angles and properties

\begin{tabular}{|l|l|}
\hline \multicolumn{1}{|c|}{ Space-time ${ }^{L} S T$} & \multicolumn{1}{c|}{ Space-time ${ }^{D} S T$} \\
\hline $\begin{array}{l}\text { The curvature }{ }^{L} S T \text { is positive } \\
\left({ }^{L} \omega>0\right)\end{array}$ & $\begin{array}{l}\text { The curvature }{ }^{D} S T \text { is negative } \\
\left({ }^{D} \omega<0\right)\end{array}$ \\
The sum of the angles of a triangle is & $-\begin{array}{l}\text { The sum of the angles of a triangle is } \\
\angle \alpha+\angle \beta+\angle \gamma>180^{\circ}\end{array}$ \\
$\begin{array}{l}\text { Space }{ }^{L} S T \text { expands (homogenous) } \\
\text { relative to }{ }^{A} S T \text { from moment } B B \text { along } \\
\text { arrow of time (Figure } 1 \text { ) to } C r\end{array}$ & $\begin{array}{l}\angle \alpha+\angle \gamma<180^{\circ} ; \\
\text { Space }{ }^{D} S T \text { contracts (heterogeneous } \\
\text { and anisotropic) relative to }{ }^{A} S T \text { from } \\
\text { moment } B B \text { to } C r \text { (Figure } 1 \text { ) }\end{array}$ \\
\hline
\end{tabular}




\subsection{Temperature and entropy}

\begin{tabular}{|c|c|}
\hline Space-time ${ }^{L} S T$ & Space-time ${ }^{D} S T$ \\
\hline $\begin{array}{l}\text { Temperature in the moment of } B B \text { is } \\
\text { hot } \\
\text { Entropy of }{ }^{L} H \text { increases along the } \\
\text { arrow of time }{ }^{L} T: \frac{\Delta^{L} H}{\Delta^{L} T}>1 \\
\text { - Entropy of }{ }^{L} H \text { is negative in } \\
\text { relationship to }{ }^{D} H\end{array}$ & $\begin{array}{l}\text { - Temperature in the moment of } B B \text { is } \\
\text { cold } \\
\text { - Entropy of }{ }^{D} H \text { increases in the time } \\
{ }^{D} T: \frac{\Delta^{D} H}{\Delta^{D}}<|-1| \\
\text { - Entropy of }{ }^{D} H \text { is negative in } \\
\text { relationship to }{ }^{L} H\end{array}$ \\
\hline
\end{tabular}

5.6 Geometry, 5th postulate and metric

\begin{tabular}{|l|l|}
\hline \multicolumn{1}{|c|}{ Space-time ${ }^{L} S T$} & \multicolumn{1}{c|}{ Space-time ${ }^{D} S T$} \\
\hline - Riemannian geometry $(\mathcal{R})$ is elliptical & $\bullet \begin{array}{l}\text { Lobachevsky-Bolyai geometry }(\mathcal{L B}) \text { is } \\
\text { hyperbolic }\end{array}$ \\
$\begin{array}{l}\text { Fifth postulate: in the plane through a } \\
\text { point that does not lie on a given } \\
\text { straight line, no line can be drawn in } \\
\text { parallel to this straight line }\end{array}$ & $\begin{array}{l}\text { Fifth postulate: in the plane through a } \\
\text { point that does not lie on a given } \\
\text { straight line, an infinite number of } \\
\text { lines can be drawn in parallel to this } \\
\text { straight line }\end{array}$ \\
$\begin{array}{l}\text { Archimedean metric. } \\
\text { In the system of measurement }(\mathrm{SI}), \text { the } \\
\text { distance is measured in } m \text { (meters). } \\
\text { The concept of Planck length } \\
\left(l_{p} \sim 10^{-34} \mathrm{~cm}\right) \text { in }{ }^{L} S T \text { requires further } \\
\text { research }\end{array}$ & $\begin{array}{l}\text { Non-Archimedean metric. } \\
\text { The definition of distance in }{ }^{D} S T \\
\text { requires further research }\end{array}$ \\
\hline
\end{tabular}

5.7 Matter, energy and information

\begin{tabular}{|c|c|}
\hline Space-time ${ }^{L} S T$ & Space-time ${ }^{D} S T$ \\
\hline $\begin{array}{l}\text { Matter }{ }^{L} M \text { : mass is positive (matter } \\
\text { density is positive) } \\
\text { The form of matter }{ }^{L} M \text { is particles } \\
\text { - Energy }{ }^{L} E \text { is positive (energy density is } \\
\text { positive) } \\
\text { - Information }{ }^{L} I \text { is stochastic }\end{array}$ & $\begin{array}{l}\text { - Antimatter }{ }^{D} M \text { : mass is negative } \\
\text { (antimatter density is negative) } \\
\text { - The form of antimatter }{ }^{D} M \text { is } \\
\text { decentralized (requires further } \\
\text { research) } \\
\text { - Energy }{ }^{D} E \text { is negative (energy density } \\
\text { is negative) } \\
\text { - Information }{ }^{D} I \text { is fractal }\end{array}$ \\
\hline
\end{tabular}


5.8 Elementary electric charge $q$ and elementary topological magnetic charge $\tilde{\theta}$

\begin{tabular}{|c|c|}
\hline Space-time ${ }^{L} S T$ & Space-time ${ }^{D} S T$ \\
\hline $\begin{array}{l}\text { - The elementary electric charge } q \text { is a } \\
\text { fundamental physical constant, a } \\
\text { quantum electric charge equal to the } \\
\text { charge of electron } e^{-}: \\
\quad q=1,602 \times 10^{-19} \mathrm{C} \text { (SI) }\end{array}$ & $\begin{array}{l}\text { The elementary topological magnetic } \\
\text { charge } \tilde{\theta}[90,91,92,93] \text { is a } \\
\text { fundamental physical fluctuating } \\
\text { parameter, with quant of magnetic } \\
\text { charge (monopole) equal to the charge } \\
\text { of a positron } \tilde{e}^{+} \text {in }{ }^{D} S T \text {. The value of } \tilde{\theta} \\
\text { is to be determined. }\end{array}$ \\
\hline $\begin{array}{l}\text { The elementary electric charge } q \text { can } \\
\text { exist in }{ }^{D} S T \text { only in the form of a dipole } \\
\tilde{q}^{-+}\end{array}$ & $\begin{array}{l}\text { Elementary topological magnetic } \\
\text { charge } \tilde{\theta} \text { can exist in }{ }^{L} S T \text { only in the } \\
\text { form of dipole } \theta^{+-}\end{array}$ \\
\hline $\begin{array}{l}\text { Coulomb's law, that determines the } \\
\text { force }{ }^{L} F \text { between two point-like electric } \\
\text { charges: } \\
\qquad{ }^{L} F=k \frac{q_{1} q_{2}}{R^{2}} \text { (where, } k=\frac{1}{4 \pi \varepsilon_{0}} \text { ) }\end{array}$ & $\begin{array}{l}\text { It is required to find law }(\mathrm{s}) \text { that } \\
\text { determine(s) the force }{ }^{D} F_{1-\infty} \text { acting } \\
\text { between two topological magnetic } \\
\text { charges } \tilde{\theta}_{1} \text { and } \tilde{\theta}_{2} \text {, equivalent to } \\
\text { Coulomb's law in }{ }^{L} S T\end{array}$ \\
\hline $\begin{array}{l}\text { - Opposite electric charges attract } \\
\text { - Similar electric charges repel }\end{array}$ & $\begin{array}{l}\text { - Opposite topological magnetic charges } \\
\text { repel } \\
\text { - Similar topological magnetic charges } \\
\text { attract }\end{array}$ \\
\hline
\end{tabular}


5.9 Electro-magnetic waves (EMW) and positron-electric waves (PEW)

\begin{tabular}{|c|c|}
\hline Space-time ${ }^{L} S T$ & Space-time ${ }^{D} S T$ \\
\hline $\begin{array}{l}\text { Electromagnetic waves }(E M W) \text { with a } \\
\text { primary elementary electric charge and a } \\
\text { secondary magnetic charge (electro- } \\
\text { magnetic interaction) }\end{array}$ & $\begin{array}{l}\text { Positron-electric waves (PEW) with a } \\
\text { primary elementary topological magnetic } \\
\text { charge and a secondary electric charge } \\
\text { (strong interaction) }\end{array}$ \\
\hline $\begin{array}{l}\text { Maxwell's equations: } \\
\operatorname{rot} \vec{E}=-\frac{\partial \vec{B}}{\partial t} \\
\vec{E}=\frac{\vec{D}}{\varepsilon_{0} \varepsilon} \\
\operatorname{rot} \vec{H}=\vec{J}+\frac{\partial \vec{D}}{\partial t} \\
\vec{H}=\frac{\vec{B}}{\mu_{0} \mu} \\
\operatorname{div} \vec{D}=\rho \\
\operatorname{div} \vec{B}=0 \\
\text { where, } \vec{E}=\text { electrical field vector } \\
\vec{D}=\text { electric displacement vector } \\
\vec{H}=\text { magnetic field vector } \\
\vec{B}=\text { magnetic displacement vector } \\
\vec{J}=\text { electric displacement current } \\
\text { density vector } \\
\rho=\text { apparent density } e \text { - charge } \\
\text { Modifications must be made to the } \\
\text { Maxwell's equations (requiring } \\
\text { definition) that take into account } \\
\text { equivalent equations describing } P E W \\
\text { in }{ }^{D} S T \\
\text { Modified Maxwell's equations must be } \\
\text { self-consistent with equivalent } \\
\text { equations in }{ }^{D} S T \text { with using the "ideal } \\
\text { exchange interaction" in }{ }^{A} S T \text { (see } \\
\text { Chapter } 7 \text {, "The nature of the spin") }\end{array}$ & $\begin{array}{l}\text { - It is required to find equations that } \\
\text { describe positron-electric waves in } \\
{ }^{D} S T \text { equivalent and self-consistent } \\
\text { with modified Maxwell's equations in } \\
{ }^{L} S T \\
\text { - Difficulties: } \\
\text { Properties of }{ }^{D} S T \text { are not well } \\
\text { researched and understood } \\
\text { Time, }{ }^{D} T \text { is not defined; approaches to } \\
\text { time measurement, }{ }^{D} T \text {, are absent; } \\
\text { the decentralized form of matter in } \\
{ }^{D} S T \text { is not defined; } \\
\text { the definition of elementary } \\
\text { topological magnetic charge } \tilde{\theta} \text { is not } \\
\text { established; } \\
\text { the distribution density of an } \\
\text { elementary topological magnetic } \\
\text { charge } \tilde{\theta} \text { on a decentralized antimatter } \\
\text { object in }{ }^{D} S T \text { is unknown }\end{array}$ \\
\hline
\end{tabular}




\subsection{Photons $\gamma$ and dark photons $\tilde{\gamma}$}

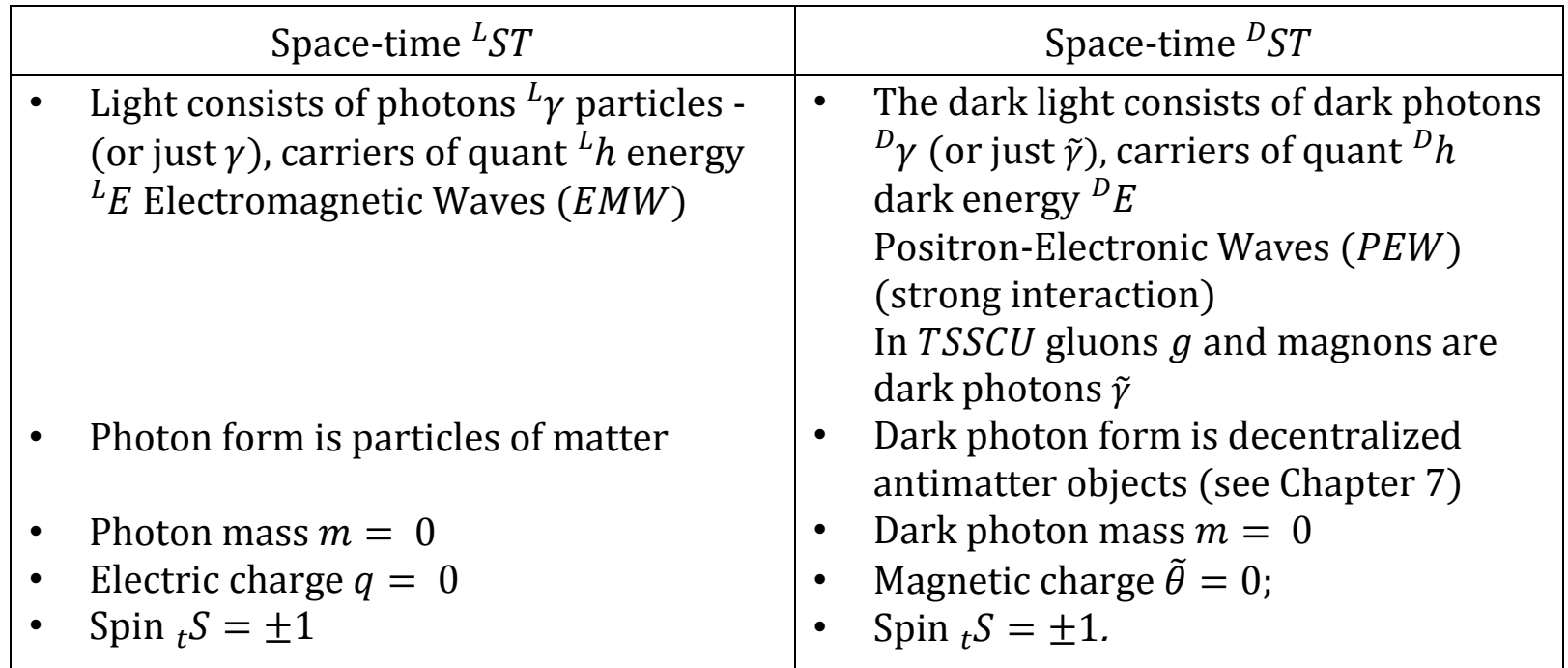

5.11 Speed of light $c$ and speed of dark light $\tilde{c}$

\begin{tabular}{|l|l|}
\hline \multicolumn{1}{|c|}{ Space-time ${ }^{L} S T$} & \multicolumn{1}{c|}{ Space-time ${ }^{D} S T$} \\
\hline $\begin{array}{l}\text { The speed of light in vacuum, } c \text {, consists } \\
\text { of photon particles } \gamma \text { and cannot } \\
\text { exceed: } c=299792458 \mathrm{~m} / \mathrm{s}\end{array}$ & $\begin{array}{l}\text { The speed of dark light } \tilde{c} \text {, consists of } \\
\text { dark photons, } \widetilde{\gamma} \text {, that require further } \\
\text { research, that takes into account } \\
\text { properties of }{ }^{D} S T \text {, metric that sets the } \\
\text { distance }{ }^{D} S \text {, time }{ }^{D} T \text {, as well as } \\
\text { approaches to determine the unit of } \\
\text { measurement, }{ }^{D} T \text {. In an algebra with } \\
\text { absolute dominant minus, it } \\
\text { corresponds to the average value: } \\
\\
\tilde{c}=-\frac{1}{c}\end{array}$ \\
\hline
\end{tabular}


Chapter 6 Three space-time model of quantum positive gravity in ${ }^{L} S T$ and multiquantum negative gravity in ${ }^{D} S T$

6.1 Universal gravitation laws $(U G L)$ in ${ }^{L} S T$ and its equivalent (anti-gravity) in ${ }^{D} S T$

\begin{tabular}{|l|l|}
\hline \multicolumn{1}{|c|}{ Space-time ${ }^{L} S T$} & \multicolumn{1}{c|}{ Space-time ${ }^{D} S T$} \\
\hline $\begin{array}{l}\text { The universal gravitation law (UGL) is: } \\
{ }^{L} F=-{ }^{L} G \frac{m_{1} m_{2}}{R^{2}}\end{array}$ & $\begin{array}{l}\text { It is necessary to find equation(s) for } \\
\text { anti-gravity law(s) which is (are) } \\
\text { equivalent to the } U G L \text { in }{ }^{L} S T:\end{array}$ \\
$\begin{array}{l}\text { negative } \\
{ }^{L} G=-6,67408 \times 10^{-11} \mathrm{~m}^{3} \mathrm{~kg}^{-1} \mathrm{C}^{-2}\end{array}$ & \\
All objects attract & ${ }^{D} F_{1-n}=(?)$ \\
\end{tabular}


6.2 General theory of relativity (GR) in ${ }^{L} S T$ and its equivalent in ${ }^{D} S T$

\begin{tabular}{|c|c|}
\hline Space-time ${ }^{L} S T$ & Space-time ${ }^{D} S T$ \\
\hline $\begin{array}{l}\text { The general theory of relativity is } \\
\qquad G_{\mu v}+\Lambda_{g_{\mu v}}=\frac{8 \pi g}{c^{4}} T_{\mu v} \\
\text { where, } G_{\mu v}=\text { Einstein tensor; } \\
\Lambda_{g_{\mu v}}=\text { cosmological constant; } \\
{ }^{L} g=\text { gravitational constant; } \\
c=\text { speed of light in vacuum; } \\
T_{\mu v}=\text { momentum energy tensor of } \\
\text { matter }{ }^{L} M ; \\
\qquad G_{\mu v}=R_{\mu v}-\frac{1}{2} g_{\mu v} R \\
\text { where, } R_{\mu v}=\text { Ricci tensor } / \text { curvature; } \\
R=\text { scalar curvature; } \\
g_{\mu v}=\text { metric }\end{array}$ & $\begin{array}{l}\text { There is a need to find system of } \\
\text { equations that satisfy the conditions } \\
\text { formulated at the end of section } 6.7 \text { of } \\
\text { this Chapter. }\end{array}$ \\
\hline $\begin{array}{l}\text { GR in TSSCU: } \\
\text { 1) } \Lambda_{g_{\mu v}}=0 \\
\text { 2) In the absence of matter, }{ }^{L} M \text { space } \\
{ }^{L} S T \text { is not empty, but consists of tissue } \\
\text { expanding in time }{ }^{L} T \\
R_{\mu v} \neq 0, R \neq 0 \text {, but the system of } \\
\text { equations, need to be supplemented, } \\
\text { while taking into account the } \\
\text { background change of curvature }{ }^{L} \omega \text { and } \\
\text { expansion of tissues of space-time (see } \\
\text { requirements formulated in end of } \\
\text { section } 6.7 \text { of this Chapter). }\end{array}$ & \\
\hline
\end{tabular}

6.3 Hubble constant ${ }^{L} H_{b}$ in ${ }^{L} S T$ and its equivalent ${ }^{D} H_{b_{1-n}}$ in ${ }^{D} S T$

\begin{tabular}{|l|c|}
\hline \multicolumn{1}{|c|}{ Space-time ${ }^{L} S T$} & \multicolumn{1}{|c|}{ Space-time ${ }^{D} S T$} \\
\hline - Based on modern science, [94, 95, 96, \\
$\begin{array}{l}\text { 97] we now know Hubble's "dynamic } \\
\text { constant" in }{ }^{L} S T \text {, which is } \\
\text { approximately equal to: } \\
{ }^{L} H_{b} \cong 67-74(\mathrm{~km} / \mathrm{s}) / \mathrm{Mpc}\end{array}$ & $\begin{array}{l}\text { In space-time }{ }^{D} S T, \text { there must exist, } \\
\text { "dynamic fluctuation parameters" } \\
{ }^{D} H_{b_{1-n}} \text {, equivalent }{ }^{L} H_{b} \text { : }\end{array}$ \\
\hline
\end{tabular}

Thus, TSSCU and the paired self-consistent parameters that it operates with, lead to new opportunities for us to build accurate mathematical models. 
6.4 Tissues of space-times ${ }^{A} S T,{ }^{L} S T$ and ${ }^{D} S T$ as real and objective components of the Universe structure

The main task of modern physics is to combine quantum physics and the $G R$, currently the best theory of gravity. To accomplish this, numerous attempts are made to develop various mathematical models of quantization of gravity or of quantum space-time that encounter insurmountable difficulties [98, 99]. However, there isn't yet an exact definition of the quantum space-time concept. It is usually considered as the appearance of discreteness in space on the Planck length scales $\left(\sim 10^{-34} \mathrm{~cm}\right)$. Therefore, we can unequivocally state that in one 6-dimensional (4 real dimensions: 3 spatial coordinates with 1 time dimension, and 2 imaginary dimensions: 1 spatial one with 1 time one) space-time, it is impossible to create the quantum property. Based on the presented hypothesis, it is necessary to add at least one more opposing and independent 6-dimensional (with equivalent 6 dimensions) space-time. With this complementarity, several conditions are required; the mutual transparency of both space-times, their heterogeneity and non-commutability, antagonism, as well as the need for constant interaction with each other. Only with strict observance of these requirements does the quantum property appears in both space-times. Therefore, each of these two independent and antagonistic space-times will have their own action quants, as a forced compromise in a constant struggle with each other. In addition to these two independent space-times, generalized Paracomplex Absolute space-time, through which all interactions occur and which is an indifferent (impartial) Arbitrator, the initial provisions described in Chapter 2 will ultimately be achieved. The main conclusion of the above is the necessity and adequacy of section 6.4 and the following statement.

Considering the fact that in the proposed theory, three space-times actively interact with each other according to given algorithms, we are forced to introduce the concept of spacetime tissues ${ }^{L} S T$ and ${ }^{D} S T$ as special-field forms of matter and antimatter containing the corresponding energy and information.

The tissue of homogenous and isotopic space-time ${ }^{L} S T$ expands (relative to the stationary tissue of ${ }^{A} S T$ ) by compressing the tissue of heterogeneous and anisotropic space-time ${ }^{D} S T$. The converse is also true - as the tissue of heterogeneous and anisotropic space-time ${ }^{D} S T$ is compressed (relative to the stationary tissue of ${ }^{A} S T$ ) due to the expansion of the tissue of homogenous and isotopic space-time ${ }^{L} S T$.

Figure 8 describes the simplest abstract model-example of flat elementary cells of the tissue of space-times ${ }^{L} S T$ and ${ }^{D} S T$ at the abstract time moment of a period $B B-C r$, originally formed from an equilateral triangle in ${ }^{A} S T$. 


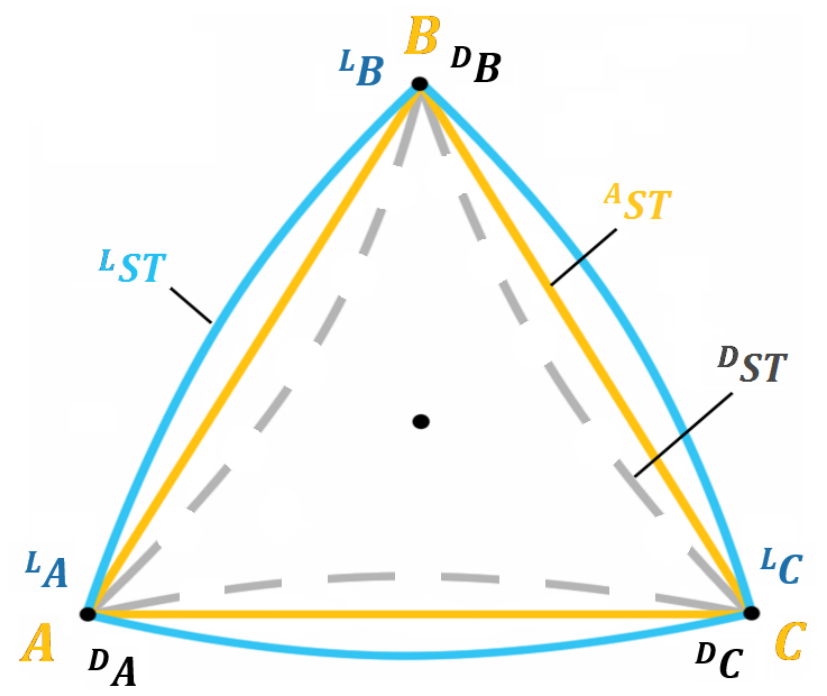

Figure 8. An abstract model-example of flat elementary cells of space-times ${ }^{L} S T$ and ${ }^{D} S T$, originally formed from an elementary cell (of a equilateral triangle) in ${ }^{A} S T$. Points $A, B, C$ are points of Absolute space-time ${ }^{A} S T$, in which, synchronously in $T_{\text {synch }}$, points ${ }^{L} A$ and ${ }^{D} A,{ }^{L} B$ and ${ }^{D} B,{ }^{L} C$ and ${ }^{D} C$ are born, forming in time ${ }^{L} T$ elementary cells of space time of positive curvature ${ }^{L} S T$, and in time ${ }^{D} T$ elementary cells of space time of negative curvature ${ }^{D} S T$, respectively. Points ${ }^{L} A$ and ${ }^{D} A,{ }^{L} B$ and ${ }^{D} B,{ }^{L} C$ and ${ }^{D} C$ are fundamentally entangled. For clarity, the cell in ${ }^{D} S T$ is depicted in the form of a broken curve, however, the form of tissues and antimatter in ${ }^{D} S T$ requires a separate study (see section 6.8 of this Chapter).

The length of the geodesic line in ${ }^{A} S T$ between points $A$ and $B$ is denoted by ${ }^{A} S_{g l}$. The length of the geodesic line in ${ }^{L} S T$ between points ${ }^{L} A$ and ${ }^{L} B$ is denoted by ${ }^{L} S_{g l}$. The generalized projection on ${ }^{A} S T$ (see section 6.7 and 6.8) of geodesic lines in ${ }^{D} S T$ between the points ${ }^{D} A$ and ${ }^{D} B$ is denoted by ${ }^{D} S_{g l}$.

Obviously, for ${ }^{A} S T$ with Euclidean geometry $(\mathcal{E})$, the geodesic line connecting the points $A$ and $B$, and the straight line (segment) connecting the points $A$ and $B$ coincide:

$$
\frac{S_{g l}}{{ }^{A} S_{g l}}=1
$$

For ${ }^{L} S T$ with Riemann geometry $(\mathcal{R})$, a geodesic line connecting the points ${ }^{L} A$ and ${ }^{L} B$ is always larger (longer) than the geodesic line connecting the points $A$ and $B$ in ${ }^{A} S T$ :

$$
\frac{{ }^{L} S_{g l}}{{ }^{A} S_{g l}}>1
$$


The value of equation 34 can be referred to as the coefficient of geodesic curvature between points ${ }^{L} A$ and ${ }^{L} B$ in ${ }^{L} S T$.

For ${ }^{D} S T$ with Lobachevsky-Bolyai geometry $(\mathcal{L B})$, the generalized projection into ${ }^{A} S T$ of the geodesic lines connecting the points ${ }^{D} A$ and ${ }^{D} B$ is always smaller (shorter) than the geodesic line connecting the points $A$ and $B$ in ${ }^{A} S T$ :

$$
\frac{\left|{ }^{D} S_{g l}\right|}{{ }^{A} S_{g l}}<1
$$

The value of equation 35 can be referred to as the coefficient of geodesic curvature between points ${ }^{D} A$ and ${ }^{D} B$ in ${ }^{D} S T$.

In accordance with formulas 33-35, between lengths ${ }^{L} S_{g l}$ in ${ }^{L} S T,{ }^{A} S_{g l}$ in ${ }^{A} S T$ and ${ }^{D} S_{g l}$ in ${ }^{D} S T$, equation 36 inequality holds:

$$
{ }^{L} S_{g l}>{ }^{A} S_{g l}>\left|{ }^{D} S_{g l}\right|
$$

The given abstract model-example of the structure of elementary cells of space-times ${ }^{L} S T$ and ${ }^{D} S T$ is the simplest. However, it is important to demonstrate that all elements of tissues in both space-times are fundamentally entangled. Physical reality is incomparably more complicated: see sections 6.6 - 6.8 of this Chapter, as well as Chapter 7. However, it becomes apparent that to build a full-fledged theory of gravity and antigravity, it is necessary to develop a theory of the physics of the structure and interactions of tissues of space-times ${ }^{L} S T$ and ${ }^{D} S T$.

\subsection{Mass formation ${ }^{L} m$ of matter particles in ${ }^{L} S T$ and mass ${ }^{D} m$ of decentralized objects of antimatter in ${ }^{D} S T$}

Hypothesis: The mass ${ }^{L} m$ of particles of matter ${ }^{L} M$ in ${ }^{L} S T$ and the mass ${ }^{D} m$ of decentralized objects of antimatter ${ }^{D} M$ in ${ }^{D} S T$ are born synchronously in $T_{\text {synch }}$ and from fundamentally entangled cells of tissues of space-times ${ }^{L} S T$ and ${ }^{D} S T$ using topological effects of entangling tissues of both spaces in ${ }^{A} S T$. These topological mechanisms require in depth research based on physical theories of braids and knots. At the same time, the formed geometric defects or deformation-distortions in the tissues of both spaces are void and compensated. But, with the movement of mass ${ }^{L} m$ particles of matter in ${ }^{L} S T$ and mass ${ }^{L} m$ of decentralized objects of antimatter in ${ }^{D} S T$, these geometrical defects, having a field nature, are an integral part of them. These statements are well coincided with $G R$ for space-time ${ }^{L} S T$. Born synchronously in $T_{\text {synch }}$, the masses ${ }^{L} m$ of particles of matter ${ }^{L} M$ in ${ }^{L} S T$ and the masses ${ }^{D} m$ of decentralized objects of antimatter ${ }^{D} M$ in ${ }^{D} S T$ are also fundamentally entangled. The ideas and hypotheses of the synchronous birth from vacuum of matter with positive mass and negative mass were periodically studied in the scientific literature [100]. However, in proposed case, postulating the birth of particles of matter and decentralized objects of 
antimatter directly from the mutually transparent tissues of space-times ${ }^{L} S T$ and ${ }^{D} S T$ with each particle of matter and the decentralized object of antimatter born in its own space-time.

Based on the proposed hypothesis, any mass ${ }^{L} m$ born in ${ }^{L} S T$ is gravitational, and any mass ${ }^{D} m$ born in ${ }^{D} S T$ is anti-gravitational, by definition. Consequently, in a stationary state, the mass ${ }^{L} m$ in ${ }^{L} S T$ aims towards rest, and the mass ${ }^{D} m$ in ${ }^{D} S T$ aims towards movement.

The most important and principal question of how particles of antimatter appear in ${ }^{L} S T$ by penetrating from ${ }^{D} S T$ is briefly discussed in Chapter 9 .

Thus, the fundamentally entangled cells of tissues of space-times ${ }^{L} S T$ and ${ }^{D} S T$ are active field basic material substances that require deep study.

An exception to the proposed mechanism in $T_{\text {synch }}$ synchronous mass formation ${ }^{L} m$ in ${ }^{L} S T$ and ${ }^{D} m$ in ${ }^{D} S T$, is the Higgs boson $H$. The next publication, "TSSCU Formalism" will describe the scalar Brout-Englert-Higgs $B E H$ field and the boson $H$ itself having spin equal to $0{ }_{t} S=$ $0)$ and their role in weak interaction.

\subsection{Self-consistent equations of the birth and evolution of space-time tissues under conditions of gravity in ${ }^{L} S T$ and antigravity in ${ }^{D} S T$}

Gravity in ${ }^{L} S T$ and antigravity in ${ }^{D} S T$ are forces that arise as opposing (tending to compensate) the steady expansion of space-time ${ }^{L} S T$ and the steady compression of spacetime ${ }^{D} S T$.

As mentioned in Chapter 3, from the moment of $B B$ there was a simultaneous change in a pair inverse quadratically dependent parameters: distance-radius of curvature ${ }^{L} S$ in the upward direction, and curvature ${ }^{L} \omega$ in the downward direction, leading to expansion of space-time ${ }^{L} S T$ and generating gravity ${ }^{L} G_{r}$. The initial values of these parameters are known: ${ }^{L} S=0$ and ${ }^{L} \omega=\infty$. Synchronous changes in these parameters must lead to the creation of continuous oriented "elliptic type" networks with a linear homogenous expansion of spacetime ${ }^{L} S T$.

From the moment of $B B$, there was a simultaneous change of pair inverse quadratically dependent parameters: distance-radius of curvature ${ }^{D} S$ in the downward direction and curvature ${ }^{D} \omega$ in the upward direction, leading to heterogeneous and anisotropic compression of space-time ${ }^{D} S T$ and generating antigravity ${ }^{D} G_{r}$. The initial values of these parameters are known: ${ }^{D} S=-\frac{1}{\infty}$, and ${ }^{D} \omega=0$. Considering that the process of compression is heterogeneous and anisotropic, synchronous changes in these parameters must lead to the creation of discontinuous rotational networks "hyperbolic type" and create topological rotational mechanism of stabilization (see section 6.7). 


\begin{tabular}{|c|c|}
\hline Space-time ${ }^{L} S T$ & Space-time ${ }^{D} S T$ \\
\hline$G_{r}=\frac{K \cdot \omega \sqrt{d x^{2}+d y^{2}+d z^{2}}}{t \cdot v}$ & $-\frac{1}{G_{r}}=\frac{\left(-\frac{1}{K}\right) \cdot\left(-\frac{1}{\omega}\right) \cdot \frac{-1}{\sqrt{-d x^{2}-d y^{2}-d z^{2}}}}{\left(-\frac{1}{t}\right) \cdot\left(-\frac{1}{v}\right)}$ \\
\hline
\end{tabular}

Equations 19L and 19D obtained in Chapter 3 are preliminary and incomplete (see section 3.7). However, they offer rich in content information for the analysis and construction of various working models of gravity in ${ }^{L} S T$ and antigravity in ${ }^{D} S T$, as well as for conducting practical experiments. It must be noted that equation 19D relates to algebra with the absolute dominant minus.

The main task at this stage is to find expansion quant of space-time ${ }^{L} S T$ that is self-consistent with dark compression quants of space-time ${ }^{D} S T$ in synchronization time $T_{\text {synch }}$.

6.7 Classification of and the new meaning of Loop Quantum Gravity ( $L Q G)$ and String Theory $(S T)$ based on the theory of mutually transparent self-consistent networks under conditions of gravity in ${ }^{L} S T$ and anti-gravity in ${ }^{D} S T$

\begin{tabular}{|c|c|}
\hline Space-time ${ }^{L} S T$ & Space-til \\
\hline $\begin{array}{l}\text { Theory of continuous oriented "elliptic } \\
\text { type" networks with linear homogenous } \\
\text { expansion under conditions of gravity }\end{array}$ & $\begin{array}{l}\text { Theory of discontinuous networks } \\
\text { "hyperbolic type" with anisotropic } \\
\text { heterogeneous compression under } \\
\text { conditions of antigravity }\end{array}$ \\
\hline $\begin{array}{l}\text { As mentioned above, gravity in }{ }^{L} S T \text { is an } \\
\text { attractive force, trying to compensate for } \\
\text { the expansion of tissues of this space, } \\
\text { related to stationary tissues of space-time } \\
{ }^{A} S T \text {. }\end{array}$ & $\begin{array}{l}\text { As mentioned above, anti-gravity in }{ }^{D} S T \text { is a } \\
\text { repulsive force, trying to compensate for } \\
\text { the compression of tissues of this space, } \\
\text { related to stationary tissues of space-time } \\
{ }^{A} S T \text {. }\end{array}$ \\
\hline $\begin{array}{l}\text { At the initial moment } B B \text {, it is minimal, and } \\
\text { reaches its maximum at the moment } C r \\
\text { (see Figure 1). Modeling space using } \\
\text { complex networks is a natural process. } \\
\text { Attempts to simulate the structure of } \\
\text { geometric cells of tissues of space-time } \\
{ }^{L} S T \text { and their interactions (based on the } \\
\text { author's interpretation) are actively carried } \\
\text { out in theory Loop Quantum Gravity }(L Q G) \\
{[101,102] \text {, where the Planck length, } l_{p} \text {, is }} \\
\text { taken as the minimal possible distance }\end{array}$ & $\begin{array}{l}\text { From the point of view of }{ }^{D} S T \text { (see Table } 3 \\
\text { of section 3.7), at the initial moment } B B \text {, } \\
\text { this force is minimal, and reaches its } \\
\text { maximum at the moment } C r \text { (see Figure 1). } \\
\text { The force of antigravity at the initial } \\
\text { moment } B B \text { breaks the actual tissue of } \\
\text { space-time, making concepts such as } \\
\text { distance, the form of antimatter, and dark } \\
\text { time }{ }^{D} T \text { subjects of further research. }\end{array}$ \\
\hline
\end{tabular}


between the nodes of the graphs, that form an elastic network structure of tissues of space-time ${ }^{L} S T$, that develops in time ${ }^{L} T$. This theory, which must be consistent with the equations of $G R$, is at its first steps. But, as mentioned in section 6.4 , to obtain the quantization effect and to further develop the future theory of gravity, it is necessary to take into account that each element of this network is fundamentally entangled with its partner in ${ }^{D} S T$, which has a nontrivial topological form. Without taking into account the fundamental entanglement of tissues of space-times ${ }^{L} S T$ and ${ }^{D} S T$, the development of any full-fledged theory of gravity becomes ineffective.
It is also necessary to consider that under the conditions of antigravity, the decomposed elements of tissues of spacetime ${ }^{D} S T$ must primarily, constantly be in motion, i.e. be waves while maintaining their topological integrity (by $t$-solitons, see section 6.8 and Chapters 7 and 8), and also preserve the property of fundamental entanglement with their partners in ${ }^{L} S T$. Both these factors, as well as the fact that the process of compression ${ }^{D} S T$ under conditions of antigravity is heterogeneous and anisotropic, must create a topological rotational mechanism that ensures stabilization and consensus of structural and functional processes in ${ }^{D} S T$ ("topological stabilization mechanism").

String Theory, which has a rich and diverse mathematical apparatus, based on the author's point of view, should be divided very accurately into two independent theories. The first one is String Network Theory (SNT), which- starts with the works of G. Veneciano [103] and I. V. Volovich [104], and tries to intuitively simulate the structure of antimatter, strong interaction, and tissues of space-time ${ }^{D} S T$ in the conditions of antigravity. From the point view of the author, the purpose of using the Calabi-Yau manifold theory in various forms is not only the compactization of hidden dimensions, but also an attempt to find such an effective rotational topological stabilization mechanism (or the meaning of manifold) under conditions of antigravity. An indirect confirmation that the CalabiYau manifolds belong to ${ }^{D} S T$ is that when it is derived, the Ricci tensor is not applicable to the Lobachevsky-Bolyai geometry and becomes equal to 0 . In order to determine the type of Calabi-Yau manifolds realized in nature and its full functionality, it is 


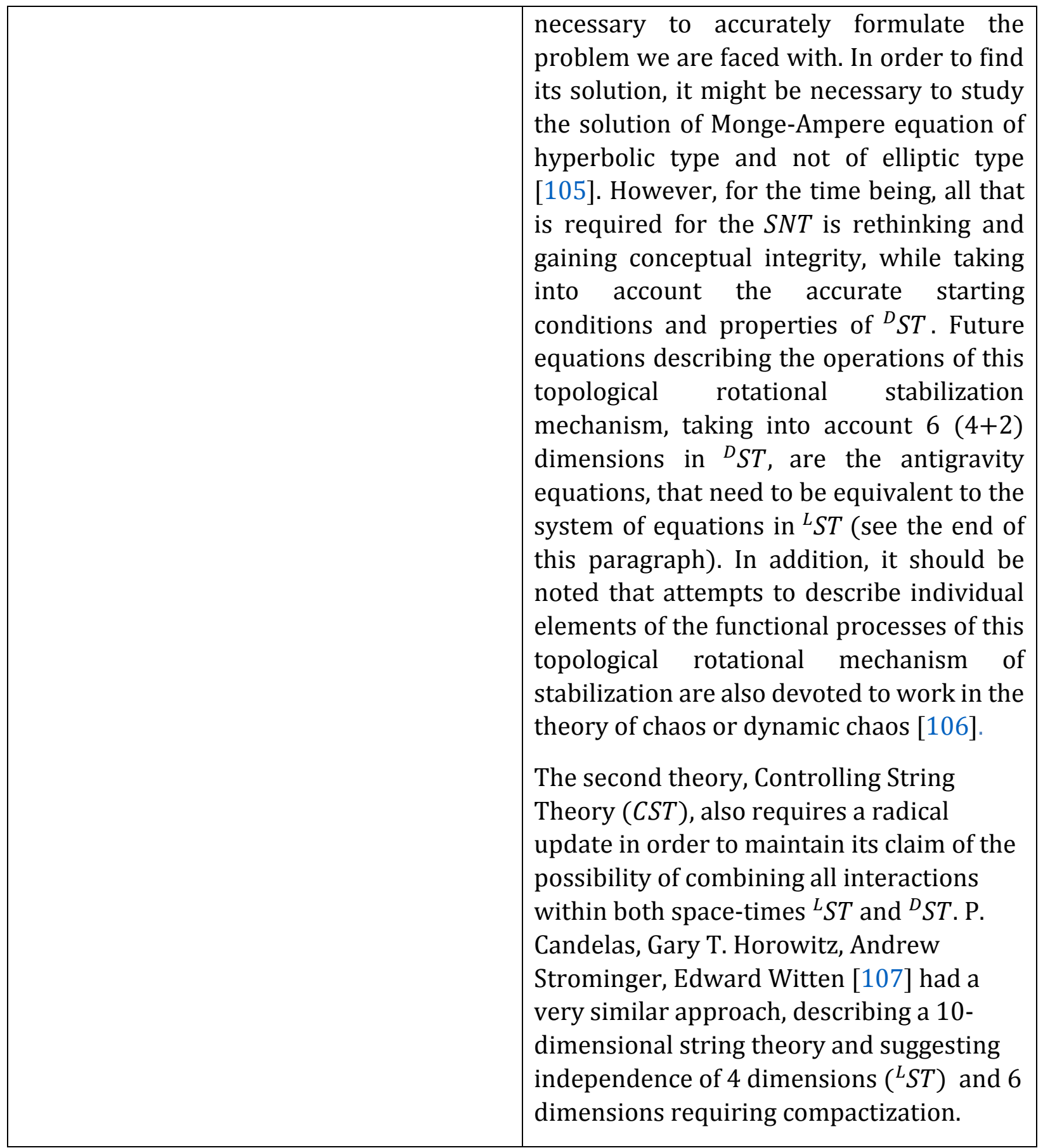

The bundle algorithms of ${ }^{A} S T$ detailed above provide completely new opportunities for the development of these two mutually complementary paths based on the theory of mutually transparent self-consistent networks having antagonistic properties. First, the presence of a primary Absolute space-time ${ }^{A} S T$ with Euclidean geometry $(\mathcal{E})$ with a reference metric and static tissue provides the future theories fundamental character. Second, the birth at the moment $B B$ and the further development of two self-consistent space-times ${ }^{L} S T$ and ${ }^{D} S T$ that have accurate dependencies. Third, the concept of fundamental entanglement and the spin effect, (see Chapter 7) imposing restrictions on the changes and properties of the pair 
"curvature and distance" that underlie the structure of space-time tissues of ${ }^{L} S T$ and ${ }^{D} S T$, suggests the future theory, mathematical rigor and protects researchers from considering abstract, unproductive options. Thus, the deliberate and purposeful development of the theory of mutually transparent self-consistent networks, as the physics of the interactions of tissues of space-times ${ }^{L} S T$ and ${ }^{D} S T$ in the foreseeable future, will solve not just the "problem of gravity quantization", but also create a fully-fledged self-consistent theory of gravity and antigravity. Given the enormous complexity of the goal, it can be divided into three parts:

1. The system of self-consistent equations in $T_{\text {synch }}$ : continuous oriented networks of the "elliptic type" with linear homogeneous extension in ${ }^{L} S T$ and discontinuous networks of the "hyperbolic type" with anisotropic heterogeneous compression in ${ }^{D} S T$ using topological rotational stabilization mechanism.

2. The system of self-consistent equations in $T_{\text {synch }}$ : the development of masses ${ }^{L} m$ of particles of matter from the "elliptic type" tissues of networks in ${ }^{L} S T$ and masses ${ }^{D} m$ of decentralized objects of antimatter from the "hyperbolic type" tissues of networks in ${ }^{D} S T$ using the topological effects of entangling the tissues of both spaces in ${ }^{A} S T$.

3. The system of self-consistent equations in $T_{\text {synch }}$ which determine changes in the parameters of the "elliptical type" network in the presence of masses ${ }^{L} m$ matter in ${ }^{L} S T$ and changes in the parameters of the "hyperbolic type" network in the presence of masses ${ }^{D} m$ decentralized antimatter objects in ${ }^{D} S T$.

6.8 The form of matter in ${ }^{L} S T$ in conditions of gravity and the form of antimatter in ${ }^{D} S T$ in conditions of antigravity.

\begin{tabular}{|c|c|}
\hline & Space- $t$ \\
\hline $\begin{array}{l}\text { Any particle of matter born in }{ }^{L} S T \text { from the } \\
\text { tissues of this space experiences the effect } \\
\text { of gravity or mutual attraction with all } \\
\text { existing objects in }{ }^{L} S T \text {. Actually, it is } \\
\text { precisely these gravitational interactions } \\
\text { that make its shape rounded, turning it into } \\
\text { a particle. The process of tissue expansion } \\
{ }^{L} S T \text { is homogeneous at large scales, but } \\
\text { fundamental entanglement with tissues } \\
{ }^{D} S T \text { forces to find various forms for local } \\
\text { expansion. In this regard, any particle } \\
\text { should take on a slight oval shape (sooner } \\
\text { or later, technologies will be developed that } \\
\text { will allow to precisely investigate particles } \\
\text { forms and this statement will be } \\
\text { researched). Depending on the tasks we are }\end{array}$ & $\begin{array}{l}\text { The properties of antigravity allow to } \\
\text { hypothesize the form of antimatter in }{ }^{D} S T \\
\text { that is equivalent to particles in }{ }^{L} S T \text {. } \\
\text { In the author's opinion, such a form } \\
\text { in }{ }^{D} S T \text { is a topological soliton, consisting of } \\
\text { a packet of elements that constantly decay } \\
\text { and move to their area of elements } \\
\text { localization (see Chapter 7). In order to } \\
\text { distinguish topological solitons as a form of } \\
\text { antimatter from secondary solitons in }{ }^{L} S T \text {, } \\
\text { they will be referred to as } t \text {-solitons, or } \\
\text { soliton clouds ( } S \text { - clouds). The following } \\
\text { reasons are the basis for this hypothesis: } \\
\text { - Antigravity acts in }{ }^{D} S T\end{array}$ \\
\hline
\end{tabular}


faced with, we can neglect such accuracy of the form of matter in micro world, for example, considering elementary particles in ${ }^{L} S T$ as point particles.
- ${ }^{D} S T$ itself is heterogeneous and anisotropic, which explains the reason for the nonlinear processes arising in it

- Time ${ }^{D} T$ in ${ }^{D} S T$ is also heterogeneous and anisotropic (see sections 5.1 and 5.3 of Chapter 5), and has the nature of a wave, which requires further research and ideas for its description in ${ }^{D} S T$ geometry $\mathcal{L B}$ is present

- The 5th postulate states that in the plane through the point that does not lie on this straight line, one can draw an infinite number of lines parallel to this straight line

- Constantly decaying and moving into an area of future localization, elements of a decentralized antimatter object behave as a single, well-controlled organism

Thus, $t$-solitons in ${ }^{D} S T$ are a form of antimatter capable of having and transferring an elementary topological magnetic charge, transferring energy, impulse, moment of impulse, etc., while maintaining their integrity. Like particles in ${ }^{L} S T, t$-solitons in ${ }^{D} S T$ can be fermions: charged or neutral, as well as bosons: neutral or charged.

Based on the above statements, any particle of matter in ${ }^{L} S T$ will be fundamentally entangled to $t$-solitons of antimatter in ${ }^{D} S T$. Our conclusion about the dualism of particles and topological soliton waves using two self-consistent space-times ${ }^{L} S T$ and ${ }^{D} S T$ is the acknowledgment of quantum mechanics basics (see Chapter 8). In particular, this approach allows us to give an exhaustive explanation to all varieties of inertia forces arising in ${ }^{L} S T$, removing the irrational term "fictitious forces" from the everyday life of physics. 


\section{Chapter 7 The three space-time nature of spin ${ }_{t} S$}

\subsection{Introduction}

Before proposing a new hypothesis about the nature of the spin and defining it, the geometric aspects of the interactions between space-times ${ }^{L} S T$ and ${ }^{D} S T$ must be considered. Based on experimental data, it has been established that, at any point (in ${ }^{L} S T$ ) the vectors of the electric $\vec{E}$ and magnetic fields $\vec{H}$ of the electromagnetic wave are mutually orthogonal. Since in TSSCU, the carriers of the primary elementary topological magnetic charges that generate the primary magnetic field are the positron and other charged $t$-solitons of antimatter forming positron-electric waves in ${ }^{D} S T$, we can assume that not only $E M W$ and $P E W$, but also other fundamental interactions between ${ }^{L} S T$ and ${ }^{D} S T$ in ${ }^{A} S T$ in $T_{\text {synch }}$ occur mutually orthogonally. Indirectly, the proposed hypothesis about the nature of complex numbers (see Chapter 3) and the use of the complex plane in mathematics confirm the same. The second hypothesis about dark complex numbers in ${ }^{D} S T$ and the dark complex plane can be interpreted in a similar way (see Chapter 3).

Based on the above, the well-known fact that confocal ellipses and hyperbolas at the intersection points are mutually perpendicular to each other is used to identify the geometric component of the nature of the spin (Figure 9).

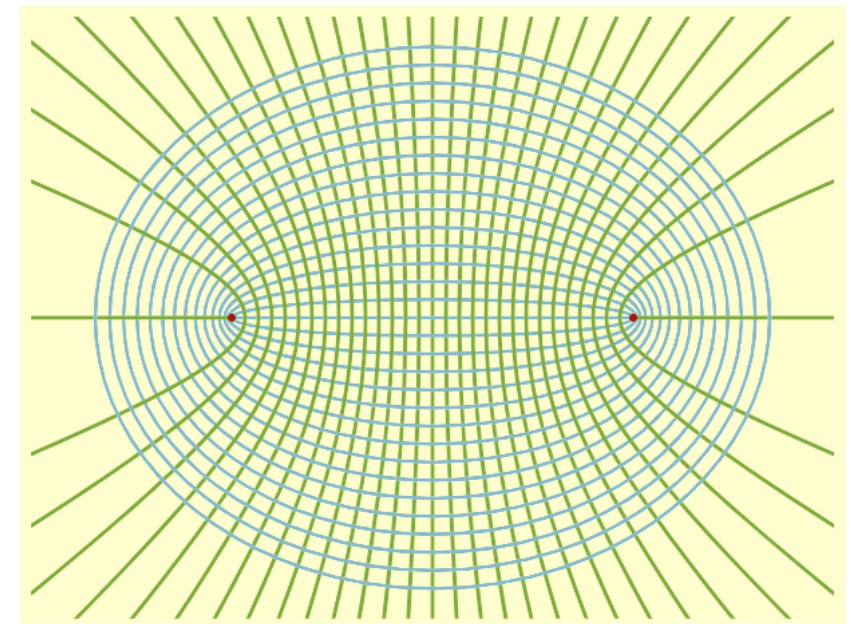

Figure 9. Confocal ellipses and hyperbolas. The wellknown mathematical fact that the intersection of confocal ellipses and hyperbolas is always mutually perpendicular serves as the basis for determining geometric component of the concept of $\operatorname{spin}{ }_{t} S$.

Looking forward, we note that the geometric arrangement of the spin ${ }_{t} S$ in ${ }^{A} S T$ at the moment $T_{\text {synch }}$ is possible only at 4 (four) points of pseudo-intersection: the trajectory of the primary (controlling) particle in ${ }^{A} S T$ - circumference; the projection of the particle trajectory ${ }^{L} M$ in ${ }^{L} S T$ into ${ }^{A} S T$ - ellipse; the projection of the confocal with the ellipse hyperboles; and, as the most important of all, possible wave paths of (see Chapter 11) 
$t$-solitons of antimatter ${ }^{D} M$ in ${ }^{D} S T$ into ${ }^{A} S T$ (Figure 10). Denote the upper left intersection by the letter $A$, the upper right intersection by the letter $B$, the lower left intersection by the letter $C$ and the lower right intersection by the letter $D$.

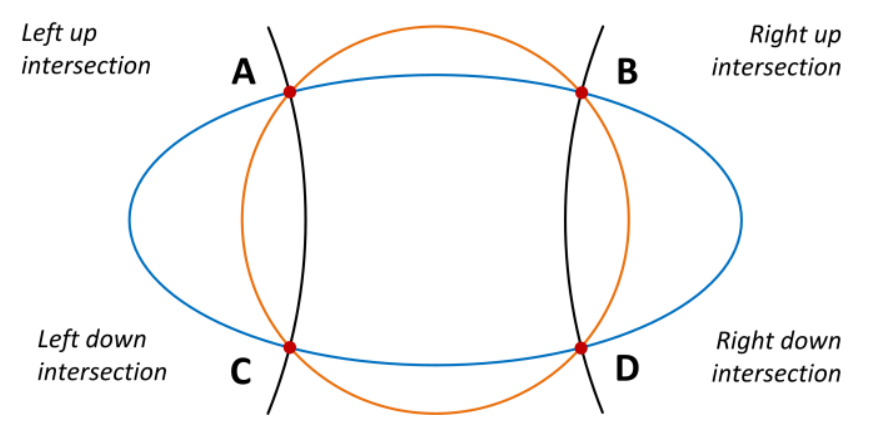

Figure 10. The four possible geometric locations of $\operatorname{spin}_{t} S$. The possible locations are points of conditional intersections in three space-times: circumference in ${ }^{A} S T$, ellipse in ${ }^{L} S T$, and projections confocal with an ellipse hyperbole in ${ }^{D} S T$ into ${ }^{A} S T$.

These 4 intersection points can also be called points of maximal convergence of projections or localization points. The next step is to consider in detail the motion of a fundamentally entangled electron in ${ }^{L} S T$ and its partner, positron, in ${ }^{D} S T$ in a self-consistent stationary atom.

\begin{tabular}{|l|l|}
\hline \multicolumn{1}{|c|}{ Space-time ${ }^{L} S T$} & \multicolumn{1}{c|}{ Space-time ${ }^{D} S T$} \\
\hline $\begin{array}{l}\text { Let an electron (particle) at a point in } \\
\text { time }{ }^{L} T \text { be in the upper left localization } \\
\text { point } A\end{array}$ & $\begin{array}{l}\text { Let a positron (particle) at a point in } \\
\text { time }{ }^{D} T \text { be in the upper left localization } \\
\text { point } A\end{array}$ \\
\hline
\end{tabular}

According to our hypothesis, localization point $A$ is the $\operatorname{spin}_{t} S_{A}$ or "transparency pass window" in ${ }^{A} S T$ in $T_{\text {synch }}$, in which "the ideal exchange interaction" occurs between electron (particle) and positron (particle), and the times ${ }^{L} T$ and ${ }^{D} T$ are synchronized. In TSSCU, the word 'ideal' is added to a known concept in physics, exchange interaction, which is only partially described $[108,109]$, without an understanding of its deep meaning. At the moment spin ${ }_{t} S$ "the ideal exchange interaction" occurs without losses (see definition of spin below).

- Further, an electron (particle) moves, for example, clockwise along a path different from a circumference, tending to elliptical, to reach localization point D

Localization point $D$ is spin ${ }_{t} S_{D}$ or "transparency pass window" in ${ }^{A} S T$ in $T_{\text {synch }}$, in which "the ideal exchange interaction" occurs between electron (particle) and positron (particle) and the times ${ }^{L} T$ and ${ }^{D} T$ are synchronized.
- Further, the positron ( $t$-soliton) moves counterclockwise along hyperbolic $t$ soliton paths, reaching localization point $D$ 
- Continuing to move clockwise along a path different from the circumference, but tending to elliptical, the electron (particle) reaches the localization point $A$ again
- Continuing counterclockwise movement along the hyperbolic $\mathrm{t}$ soliton paths, the positron ( $t$-soliton) reaches the localization point $A$ again

For one revolution in an orbit, different from the circumference, but tending to elliptical in ${ }^{L} S T$, the projection of the electron into ${ }^{A} S T$ is twice as close as possible to the projection of the positron into ${ }^{A} S T$. Positron, making one revolution along hyperbolic $t$-soliton paths in ${ }^{D} S T$ is also twice as close as possible to the projection of the electron into ${ }^{A} S T$. Thus, the spin of the electron and positron, each moving in their own space-time from the point of maximal convergence $A$ to the point of maximal convergence of projections $D$, and vice versa ${ }_{t} S_{(A \leftrightarrow D)}= \pm \frac{1}{2}$, as it should be with fermions.

\subsection{General definition of a $\operatorname{spin}_{t} S$}

Spin ${ }_{t} S$ is a "transparency pass window" arising in the Absolute space-time ${ }^{A} S T$ in $T_{\text {synch }}$ between a particle of matter ${ }^{L} M$ by a fermion (or by a particle-carrier of interactions which is a boson) in space-time ${ }^{L} S T$ and its partner particle of antimatter ${ }^{D} M$ by a fermion (or by a particle-carrier of interactions which is a dark boson) in space-time ${ }^{D} S T$, when between fundamentally entangled particle of matter ${ }^{L} M$ and its partner, a particle of antimatter ${ }^{D} M$ the following become possible:

- Ideal (lossless) exchange interaction

- Synchronization of time ${ }^{L} T$ and ${ }^{D} T$

It should be noted that the ideal exchange interaction for fermions and bosons has significant differences, which will be discussed below.

In TSSCU, for the motion of fundamentally entangled massless bosons and free fermions (outside the atom or nucleus), in addition to stationary closed orbits introduced by J. Kepler in 1609, oscillating non-closed orbits will also be considered (see sections 7.3.3 and 7.4.2).

Thus, spin ${ }_{t} S$ is measured by the number of moments of maximal convergence of projections, fundamentally entangled particles of matter ${ }^{L} M$ in space-time ${ }^{L} S T$, and their partners: $t$-solitons of antimatter ${ }^{D} M$ in space-time ${ }^{D} S T$ into Absolute space-time ${ }^{A} S T$ in $T_{\text {synch }}$ in one revolution ( $2 \pi$ ) of matter particles ${ }^{L} M$ in orbits in space-time ${ }^{L} S T$, and, accordingly, in one revolution of $(2 \pi) t$-solitons of antimatter ${ }^{D} M$ in orbits in space-time ${ }^{D} S T$.

$$
\operatorname{Spin}_{t} S=\frac{\text { one revolution of matter particles }{ }^{L} M \text { and } t-\text { solitons }{ }^{D} M \text { in their orbits }}{\text { number of moments of projection of maximal convergence }}
$$


It should be noted that $t$-solitons of antimatter ${ }^{D} M$ in ${ }^{D} S T$ at the moment of spin ${ }_{t} S$ will always be localized into a particle, since in ${ }^{A} S T$ Euclidean geometry $(\mathcal{E})$ with constant gravity ${ }^{A} G_{r}=1$ is realized.

\subsection{The nature of spin fermions}

\subsubsection{General positions and definition}

Spin fermions ${ }_{t} S= \pm \frac{1}{2}$, i.e. for one revolution $(2 \pi)$ in their orbits, the projections of particles of matter ${ }^{L} M$ in space-time ${ }^{L} S T$, and, accordingly, $t$-solitons of antimatter ${ }^{D} M$ in space-time ${ }^{D} S T$, are twice as close as possible to each other:

$$
\text { Spin }{ }_{t} S=\frac{\text { one revolution of fermion matter }{ }^{L} M \text { and }{ }^{D} M \text { in their orbits }}{\text { number of moments of maximal convergence of projections }}= \pm \frac{1}{2}
$$

In modern physics, spin ${ }_{t} S= \pm \frac{1}{2}$ is measured in units $\hbar$, which is not entirely correct. In fact, taking into account the formula: ${ }^{L} E=\hbar \omega$ (where $\hbar=\frac{h}{2 \pi}$, and $\omega=2 \pi f, h$ is the Planck constant, $\hbar$ is Dirac constant, $f$ is the frequency, $\omega$ is the cyclic or angular frequency), this means that the energy of fermions in ${ }^{L} S T$ and ${ }^{D} S T$ in one revolution $(2 \pi)$ around their orbits is updated twice (with consideration of new interpretation of quantum mechanics (see Chapter 11), author believes using the simple formula ${ }^{L} E=\hbar \omega$ for fermions to be correct).

During the ideal exchange interaction fermions have the following five operations:

1. The elementary point electric charge of an electron in ${ }^{L} S T$ is converted into a dipole in ${ }^{D} S T$ (i.e., the positron in ${ }^{D} S T$ has its own electric moment).

2. The elementary topological magnetic charge of positron in ${ }^{D} S T$ is converted into dipole in ${ }^{L} S T$ (i.e., the electron in ${ }^{L} S T$ has its own magnetic moment).

3. The electron and positron exchange their impulses ${ }^{L} P$ and ${ }^{D} P$.

4. The electron and positron exchange their stochastic ${ }^{L} I$ and fractal ${ }^{D} I$ information, accordingly.

5. A synchronization of times ${ }^{L} T$ and ${ }^{D} T$ occurs.

Such an ideal exchange interaction with "vital" parameters between fermion in ${ }^{L} S T$ and its fundamentally entangled partner in ${ }^{D} S T$ does not allow two or more fermions in ${ }^{L} S T$ and ${ }^{D} S T$ to be in the same quantum state (Pauli principle), thereby, giving the fermions the property to form a structure of matter.

As known, there are $2{ }_{t} S \mid+1$ spin states. Therefore, in fermions, only 2 states are possible: $+\frac{1}{2}$ and $-\frac{1}{2}$.

The division of spin values by the positive and negative signs ${ }_{t} S= \pm \frac{1}{2}$, and concept of helicity (requires an accurate definition) and chirality are not the subject of this article and can be considered in one of the subsequent publications. In all cases, formulas are required 
to describe the motion of $t$-solitons in ${ }^{D} S T$, as well as formulas describing the concerted motion of both fundamentally entangled fermions (see Chapter 11).

Taking into account the fundamentally entangled fermions that make up the self-consistent atom move in stationary closed elliptical and hyperbolic $t$-soliton orbits in opposite radial directions (Figures 11-14), and the free fundamentally entangled fermions (outside the atom or nucleus) move along oscillating non-closed elliptical and hyperbolic $t$-soliton orbits (Figure 15), all the possible combinations can be considered.

\subsubsection{Geometric spin arrangement of fundamentally entangled fermions in a self-consistent} atom

In a case when, for observer $\mathrm{N} 1$, an electron moves clockwise in an elliptical orbit the positron ( $t$-soliton) will move counterclockwise (for example, in the hydrogen atom, in the $s$-orbital) along the hyperbolic $t$-soliton paths (Figures 11 and 12). The only possible geometric combination in this case, in which the spin effect is possible, will be the upper left localization point $A$ and the lower right localization point $D$, which can be written as ${ }_{t} S_{(A \leftrightarrow D)}= \pm \frac{1}{2}$.
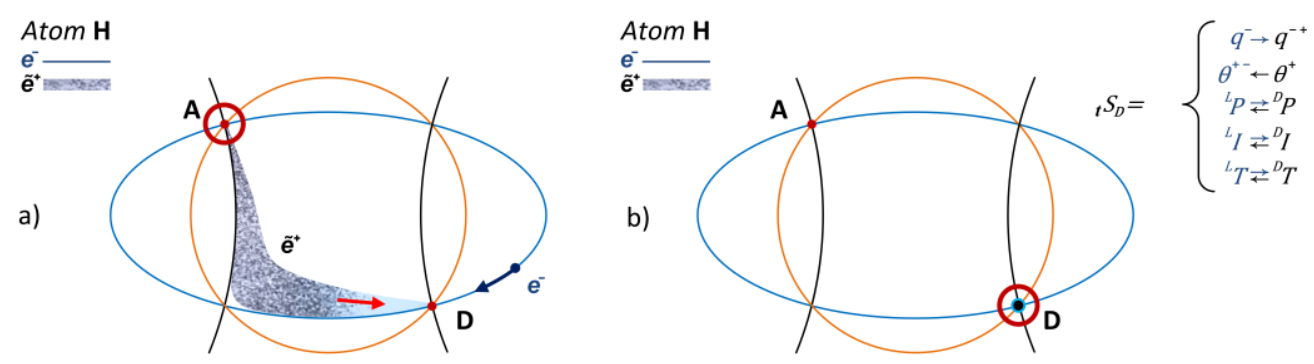

Figure 11 ( $a$ and $b$ ). The concept of motion of fundamentally entangled fermions in a selfconsistent particle-soliton hydrogen atom of protium. Figure 11a, on the left, shows how an electron (particle) moves clockwise, along a trajectory different from circumference, tending to elliptical in ${ }^{L} S T$, and a positron ( $t$-soliton) that moves counterclockwise (along $s$-orbital) in hyperbolic $t$ - soliton trajectories in ${ }^{D} S T$, approaching localization point $D$ $\left({ }_{t} S_{D}= \pm \frac{1}{2}\right)$ in ${ }^{A} S T$. Figure $11 \mathrm{~b}$, on the right, shows the moment of maximal convergence of the projections of the electron (particle) and positron (particle) at the bottom right localization point $D\left({ }_{t} S_{D}= \pm \frac{1}{2}\right)$ in ${ }^{A} S T$, when the ideal exchange interaction becomes possible between electron and positron particles. 

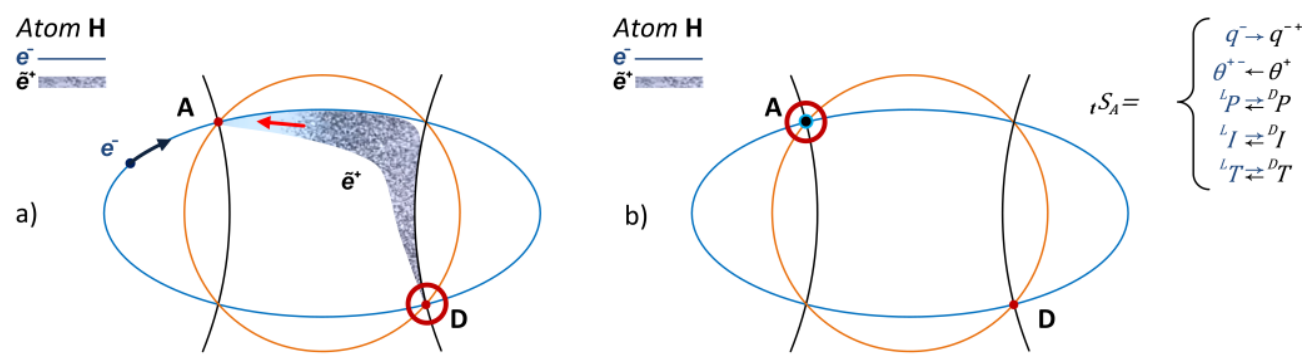

Figure 12 ( $a$ and $b$ ). The concept of motion of fundamentally entangled fermions in a selfconsistent particle-soliton hydrogen atom of protium. Figure 12a, on the left, shows how an electron (particle) moves clockwise, along a trajectory different from circumference, tending to elliptical in ${ }^{L} S T$, and a positron ( $t$-soliton) that moves counterclockwise (along $s$-orbital) in hyperbolic $t$ - soliton trajectories in ${ }^{D} S T$, approaching localization point $A$ $\left({ }_{t} S_{A}= \pm \frac{1}{2}\right)$ in ${ }^{A} S T$. Figure $12 \mathrm{~b}$, on the right, shows the moment of maximal convergence of the projections of the electron (particle) and positron (particle) at the upper left localization point $A\left({ }_{t} S_{A}= \pm \frac{1}{2}\right)$ in ${ }^{A} S T$, when the ideal exchange interaction becomes possible between electron and positron particles.

For observer N2, who is opposite of observer N1, an electron moves counterclockwise in an elliptical orbit and the positron ( $t$-soliton) moves clockwise (for example, in $s$-orbital), along the hyperbolic $t$-soliton paths (see Figures 13 and 14). The only possible geometric combination in this case, in which the spin effect is possible, will be the upper right localization point $B$ and the lower left localization point $C$, that can be written as ${ }_{t} S_{(B \leftrightarrow C)}= \pm \frac{1}{2}$
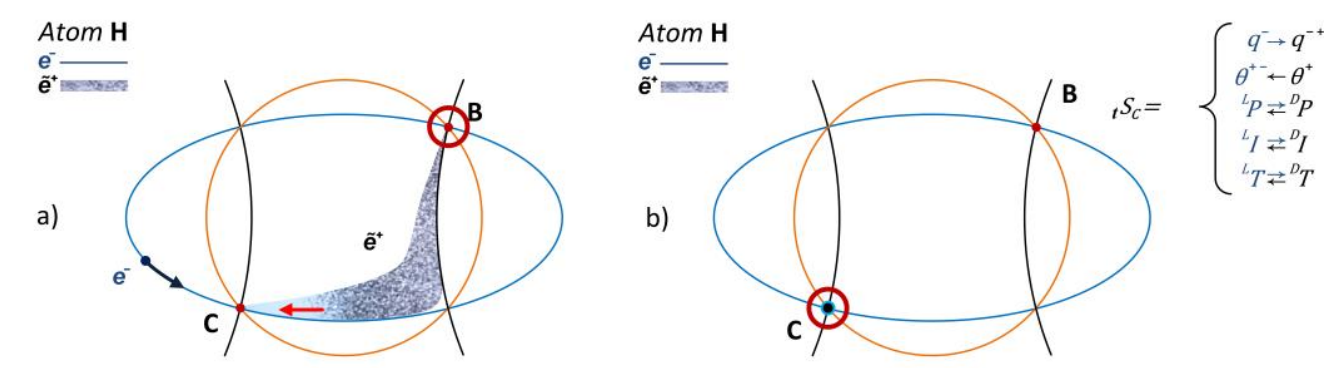

Figure 13 ( $a$ and $b$ ). The concept of motion of fundamentally entangled fermions in a selfconsistent particle-soliton hydrogen atom of protium. Figure 13a, on the left, shows how an electron (particle) moves counterclockwise, along a trajectory different from circumference, tending to elliptical in ${ }^{L} S T$, and a positron ( $t$-soliton) that moves clockwise (along $s$-orbital), in possible hyperbolic $t$ - soliton trajectories in ${ }^{D} S T$, approaching localization point $C\left({ }_{t} S_{C}= \pm \frac{1}{2}\right)$ in ${ }^{A} S T$. Figure 13b, on the right, shows the moment of maximal convergence of the projections of the electron (particle) and positron (particle) at the bottom left localization point $C\left({ }_{t} S_{C}= \pm \frac{1}{2}\right)$ in ${ }^{A} S T$, when the ideal exchange interaction becomes possible between electron and positron particles. 

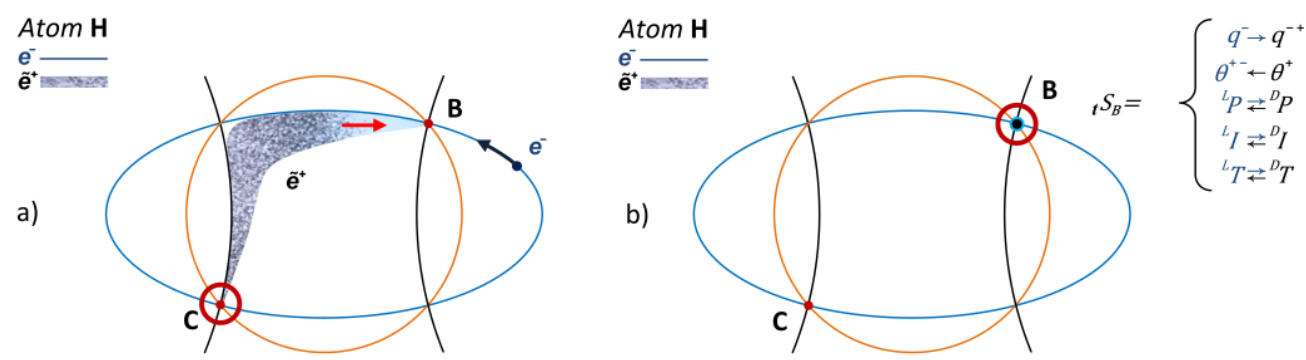

Figure $14(a$ and $b)$. The concept of motion of fundamentally entangled fermions in a selfconsistent particle-soliton hydrogen atom of protium. Figure 14a, on the left, shows how an electron (particle) moves counterclockwise, along a trajectory different from circumference, tending to elliptical in ${ }^{L} S T$, and a positron ( $t$-soliton) that moves clockwise (along $s$-orbital) in possible hyperbolic $t$ - soliton trajectories in ${ }^{D} S T$, approaching localization point $B\left({ }_{t} S_{B}= \pm \frac{1}{2}\right)$ in ${ }^{A} S T$. Figure $14 \mathrm{~b}$, on the right, shows the moment of maximal convergence of the projections of the electron (particle) and positron (particle) at the upper right localization point $B\left({ }_{t} S_{B}= \pm \frac{1}{2}\right)$ in ${ }^{A} S T$, the ideal exchange interaction becomes possible between electron and positron particles.

Thus, provided in Figures 11-12 and 13-14 are examples of the movement of fundamentally entangled electrons and positrons (along $s$-orbital) in self-consistent particle-soliton atom that are left-right symmetrical.

\subsubsection{Geometric spin arrangement of fundamentally entangled free fermions during the motion} (outside the atom or nucleus) along oscillating non-closed orbits

Free fundamentally entangled fermions outside an atom or nucleus move in concert, each in its own space-time along oscillating non-closed orbits. In the simplest one-dimensional case, such oscillating non-closed elliptical orbits in ${ }^{L} S T$ can be defined as trajectories, consisting of curves, whose reflection using mirror symmetry (the required number of times) leads to close the orbit onto itself (see Figures 15). Further research and precise formulas are required to determine complex oscillating non-closed hyperbolic $t$-soliton orbits in ${ }^{D} S T$. However, the main concept is that, for the spin effect realization, $t$-solitons always move from one localization point to another. Therefore, a pair of localization points of oscillating fundamentally entangled fermions ${ }_{t} S_{A \leftrightarrow C}= \pm \frac{1}{2}$ or ${ }_{t} S_{B \leftrightarrow D}= \pm \frac{1}{2}$ will be in new areas of spacetimes ${ }^{A} S T,{ }^{L} S T$ and ${ }^{D} S T$ each time.

It is necessary to make a reservation that in Figure 15 ( $a$ and $b$ ) presented below, only the conception of two possible hyperbolic $t$-soliton orbits for a positron using one branch of a hyperbola having different helicity is shown. 


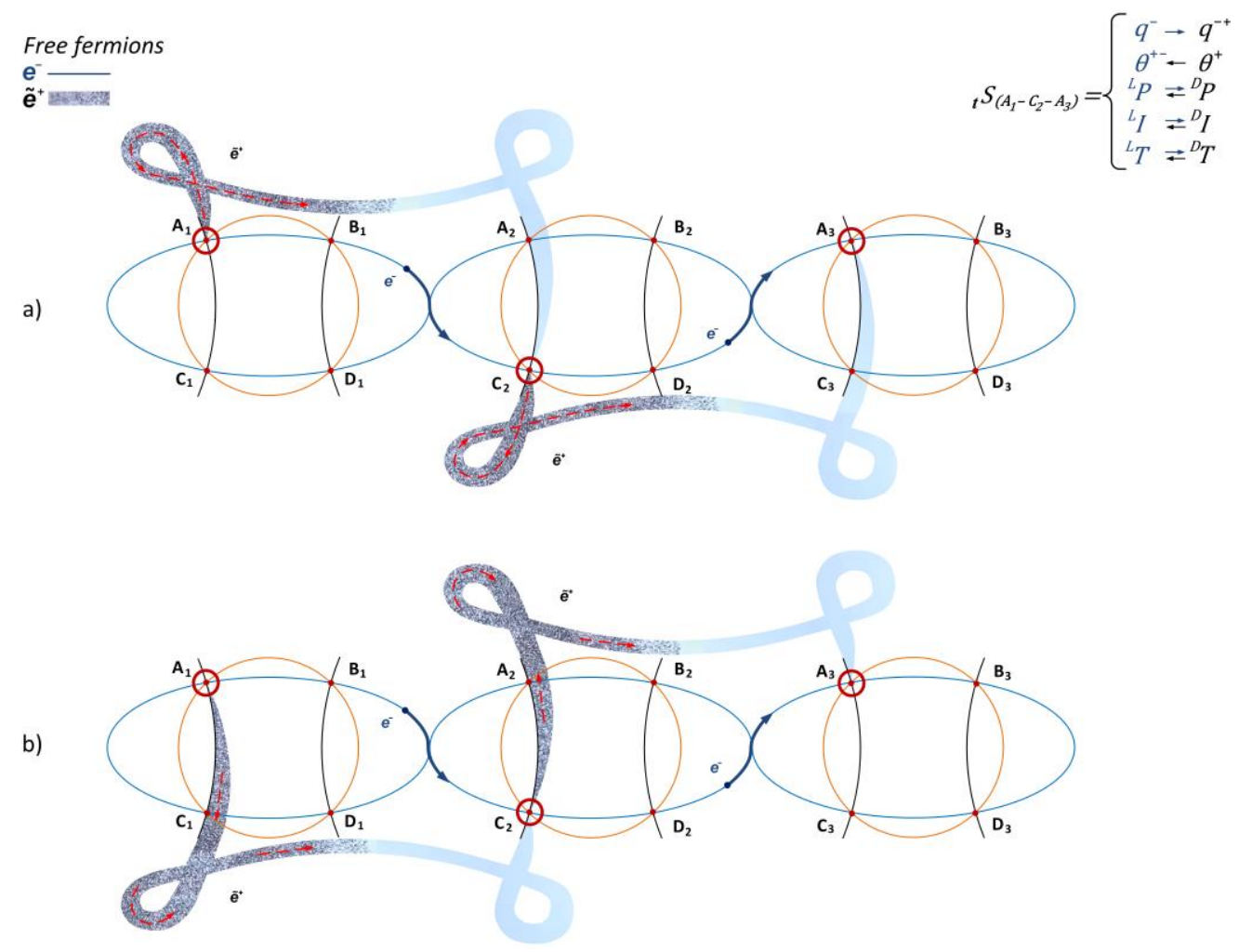

Figure 15 ( $a$ and $b$ ). The concept of motion of free (outside an atom or nucleus) fundamentally entangled fermions: an electron (particle) moving along oscillating non-closed elliptical orbits and a positron ( $t$-soliton) along two possible oscillating non-closed hyperbolic $t$-soliton orbits in ${ }^{D} S T$, using one branch of hyperbola. Each of the localization points $A_{1}, C_{2}$ and $A_{3}$ is a transparency window or spin ${ }_{t} S$, when the ideal exchange interaction between electron particle and positron particle becomes possible. In both $15 \mathrm{a}$ and $15 \mathrm{~b}$, trajectories of electron in ${ }^{L} S T$ coincide. Figure $15 \mathrm{a}$ shows positron's motion ( $t$-soliton) in ${ }^{D} S T$ along oscillating non-closed possible hyperbolic $t$-soliton orbit from localization point $A_{1}$ to localization point $C_{2}$ and then to localization point $A_{3}$ (with 2 loops per half turn), in which "helicity" can be interpreted as positive. Figure $15 \mathrm{~b}$ shows positron's motion ( $t$-soliton) in ${ }^{D} S T$ along oscillating non-closed possible hyperbolic $t$-soliton orbit from localization point $A_{1}$ to localization point $C_{2}$ and then to localization point $A_{3}$ (with 2 loops per half turn), in which "helicity" can be interpreted as negative.

It should be noted that the trajectories of free fundamentally entangled fermions: electron and positron, are not left-right symmetrical.

\subsection{The nature of spin bosons}

\subsubsection{General positions and definition}

The bosons are divided into two classes: massless bosons (photons in ${ }^{L} S T$ and dark photons in ${ }^{D} S T$ ) without charges, and massive vector bosons $\left({ }^{+} W,{ }^{-} W,{ }^{0} Z\right)$ among which ${ }^{ \pm} W$ have a charge. The spin of all bosons is ${ }_{t} S= \pm 1$, but the ideal exchange interaction varies significantly between massless neutral and massive, having charges bosons. Next, we will 
focus on the massless neutral bosons, since ${ }^{ \pm} W$, those arising by weak interaction, are an exception to the rule and will be discussed at the end of this section.

The spin of the bosons is ${ }_{t} S= \pm 1$, i.e., in one revolution $(2 \pi)$ in their oscillating non-closed orbits, the projections of fundamentally entangled particles of matter ${ }^{L} M$ in space-time ${ }^{L} S T$, and accordingly, the $t$-solitons of antimatter ${ }^{D} M$ in space-time ${ }^{D} S T$, once come as close as possible to each other:

Spin ${ }_{t} S=\frac{\text { one revolution of bosons of matter }{ }^{\mathrm{L}} \mathrm{M} \text { and of dark bosons }{ }^{\mathrm{D}} \mathrm{M} \text { in their orbits }}{\text { number of moments of maximal convergence of projections }}= \pm 1$

In modern physics, spin ${ }_{t} S= \pm 1$ is measured in units $\hbar$, which is not entirely correct. In fact, given the formula: ${ }^{L} E=\hbar \omega$ (where $\hbar=\frac{h}{2 \pi}$, and $\omega=2 \pi f, h$ is the Planck constant, $\hbar$ is the Dirac constant, $f$ is the frequency, $\omega$ is the cyclic or angular frequency), this means that the energy of massless neutral bosons per revolution $(2 \pi)$ around its orbits does not change.

During an ideal exchange interaction, the following two operations take place for a photon $\gamma$ and its fundamentally entangled dark photon $\tilde{\gamma}$ :

1. The bosons exchange their stochastic ${ }^{L} I$ and fractal ${ }^{D} I$ information, accordingly.

2. Synchronization of times ${ }^{L} T$ and ${ }^{D} T$ occurs.

Massless bosons, unlike charged fermions, do not have charges and do not exchange their impulses ${ }^{L} P$ and ${ }^{D} P$. These differences and $\operatorname{spin}{ }_{t} S= \pm 1$ allow many massless neutral bosons in ${ }^{L} S T$ to be in the same quantum state, thereby endowing them with the property of being carriers of interactions.

In TSSCU, photons $\gamma$ and dark photons $\tilde{\gamma}$ are fundamentally entangled.

Proceeding from the proposed hypothesis of the nature of the spin (section 5.10 and 5.11 of chapter 5) and based on the above-discussed conclusions, the following predictions can be confidently made:

- Particles, which are antiparticles of themselves, do not exist

- Massless neutral boson photon $\gamma$ in space-time ${ }^{L} S T$ with $\operatorname{spin}{ }_{i} S= \pm 1$, must have a fundamentally entangled partner: massless neutral dark photon $\tilde{\gamma}$ in space-time ${ }^{D} S T$ as well as with $\operatorname{spin}{ }_{i} S= \pm 1$

In this case, photons $\gamma$ are carriers of quants ${ }^{L} \hbar$ of electromagnetic interaction, and dark photons $\tilde{\gamma}$ are carriers of dark quants ${ }^{D} \hbar_{1-n}$ of positron-electric (strong) interaction. The hypothesis (possibility) of a photon having wave function was suggested (reviewed) as early as 1930 [110, 111], but ran into serious theoretical difficulties. The discovery of optical solitons [112] allows to hope that the problems of calculating the self-consistent motion of photons and dark photons will be solved in the near future.

Based on the knowledge that there are $2\left|{ }_{t} S\right|+1$ spin states, bosons have 3 possible states: $+1,-1$, and the so-called 0 . Division of the spin value by positive and negative signs ${ }_{t} S=$ \pm 1 , as well as an accurate definition of the concept of helicity will become clear only after 
obtaining the final formulas describing the consistent motion of fundamentally entangled bosons. The following examples of their possible consistent movement should be considered.

\subsubsection{The geometric spin position of fundamentally entangled massless bosons while moving along oscillating unclosed orbits}

When a fundamentally entangled photon particle moves in ${ }^{L} S T$ out of 4 possible geometrical points of the spin's location (see Figure 10), in one direction, it passes a large part of the elliptical trajectory (along a given ellipse), and in the other direction a smaller part of the elliptical trajectory (along this ellipse), see Figure 16 ( $a$ and $b$ ). Taking into account the spin ${ }_{t} S= \pm 1$, photon $\gamma$ in ${ }^{L} S T$ travels one revolution along an oscillating non-closed elliptical trajectory to the next point of localization and ideal exchange interaction.

It is necessary to note that Figure 16 ( $a$ and b) presented below is shown solely as a conception of two possible hyperbolic $t$-soliton orbits for the dark photon, $\tilde{\gamma}$, using the branches of both hyperbolas that have different helicity.

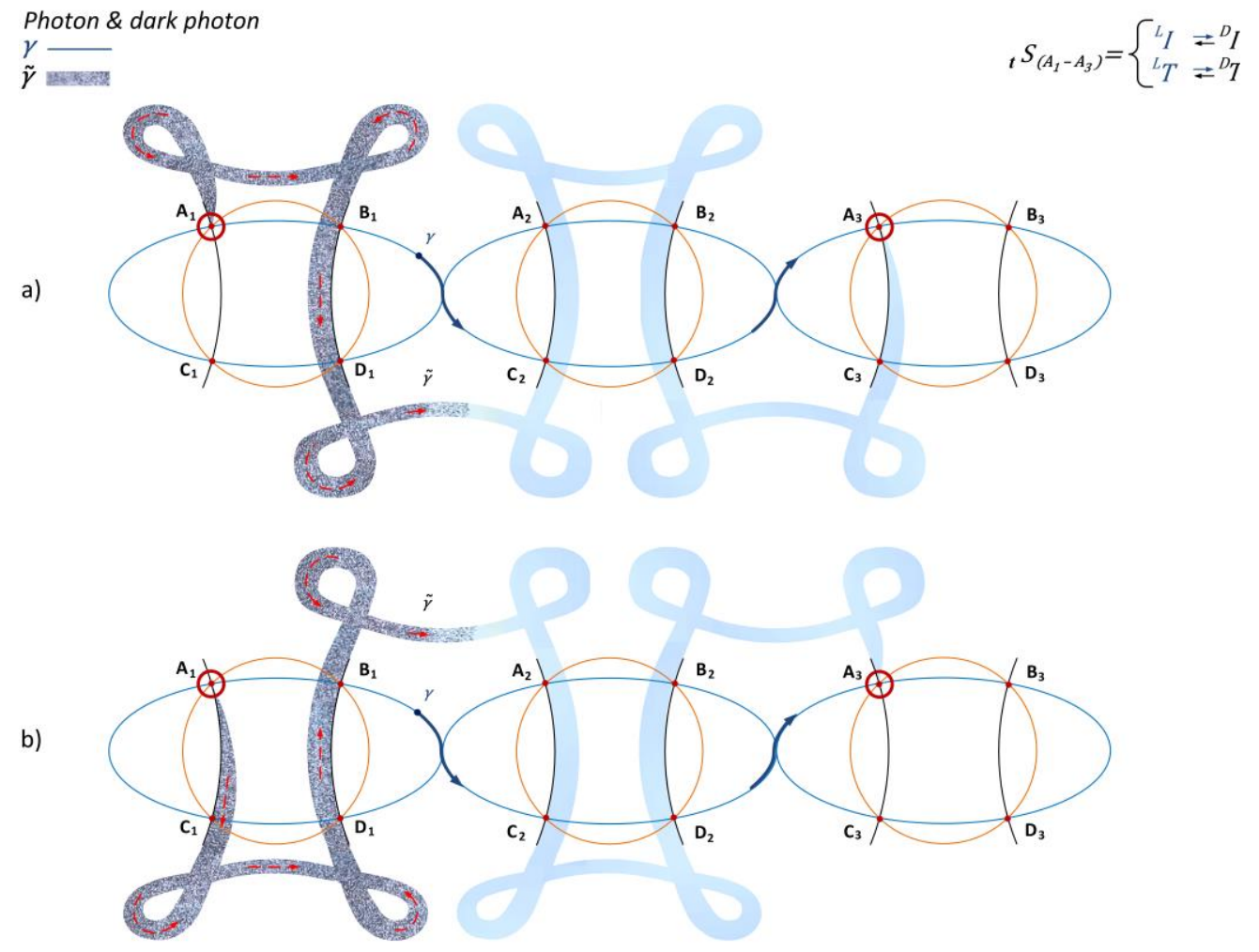

Figure 16 ( $a$ and $b$ ). The concept of motion of fundamentally entangled bosons: a photon (particle) moves along oscillating non-closed elliptical orbit in ${ }^{L} S T$ and dark photons ( $t$ soliton) along two possible oscillating non-closed hyperbolic $t$-soliton orbits in ${ }^{D} S T$ using branches of both hyperbolas. Localization points $A_{1}$ and $A_{3}$ are transparency windows or spin ${ }_{t} S$, when the ideal exchange interaction between photon particle and dark photon particles becomes possible. On both parts of Figure 16 (a and b) in both 16a and 16b photon trajectories coincide. Figure 16 ( $a$ and $b$ ) shows motion of dark photon ( $t$-soliton) in ${ }^{D} S T$ along two different oscillating non-closed possible hyperbolic $t$-soliton orbits from localization point $A_{1}$ to localization point $A_{3}$ that have different helicity. 
It is important to notice that the motion of fundamentally entangled bosons: photon and dark photon, is not left-right symmetrical.

The third state of the $\operatorname{spin}{ }_{t} S$, boson the so-called 0 , is forbidden for photons and dark photons. The reason for this prohibition will be discussed in the next article, "TSSCU Formalism".

\subsubsection{Spin features when fundamentally entangled massive vector ${ }^{ \pm} \mathrm{W}$ bosons are in motion}

For charged ${ }^{ \pm} W$ massive vector bosons, the ideal exchange interaction will occur similarly to the way it occurs in fermions, with the only significant difference being that ${ }^{ \pm} W$ bosons it occurs once per revolution around its orbits. This difference makes charged massive vector ${ }^{ \pm} W$ bosons unstable and short-lived, which allows them to play their proper role as carriers of weak interaction.

\subsubsection{Spin features of a massive vector ${ }^{0} Z$ boson. Prediction.}

A neutral massive vector ${ }^{0} Z$ boson, in which the ideal exchange interaction should occur with its fundamentally entangled partner from antimatter should be considered. However, its partner, a dark boson ${ }^{0} \tilde{Z}$, from antimatter has yet to be found. The main conclusion is that the nature of the spin hypothesis excludes this possibility. Based on this, the following predictions can be confidently made:

- $\quad$ Particles, which are antiparticles of themselves, do not exist.

- Massive boson ${ }^{0} Z$ in space-time ${ }^{L} S T$ with $\operatorname{spin}{ }_{i} S= \pm 1$, must have a fundamentally entangled partner of a neutral massive boson from antimatter ${ }^{0} \tilde{Z}$ in space-time ${ }^{D} S T$ as well as with $\operatorname{spin}_{i} S= \pm 1$.

\subsubsection{Spin of the Higgs boson $H$}

The features of the Higgs boson $H$, which has zero spin $\left({ }_{i} S=0\right)$, and the Brout-Englert-Higgs $(B E H)$ scalar field will be discussed in the next article, "TSSCU Formalism".

\subsection{Conclusion}

Spin ${ }_{t} S$ is a fundamental mechanism or effect that ensures ideal exchange interaction and synchronization of times ${ }^{L} T$ and ${ }^{D} T$ between fundamentally entangled particles in ${ }^{L} S T$ and their partners, $t$-solitons in ${ }^{D} S T$. 


\section{Chapter 8 A self-consistent particle-soliton model of a hydrogen atom (protium) in the three space-times ${ }^{A} S T,{ }^{L} S T,{ }^{D} S T$}

When discussing the self-consistent particle-soliton model of the simplest hydrogen atom, protium, there is a need to take into account that a protium does not contain neutral particles but consists of a nucleus and an elementary particle moving around it.

For each of the three space-times, the model describing a protium will have its own significant differences. These differences are due to the properties of these space-times, as well as the fundamental spin mechanism.

In absolute space-time ${ }^{A} S T$, the atom of the primary (or controlling) protium is a primary electron that moves in an ideal circumference around a primary proton consisting of three primary quarks, since Euclidean geometry and constant gravity laws are present in ${ }^{A} S T$.

\begin{tabular}{|c|c|}
\hline Space-time ${ }^{L} S T$ & Space-time ${ }^{D} S T$ \\
\hline $\begin{array}{l}\text { The atom and nucleus of protium } \\
\text { include a proton, } p \text {, consisting of three } \\
\text { quarks: }(u, u, d) \text { and an electron, } e \text { that } \\
\text { moves in an orbit different from the } \\
\text { circumference, tending towards } \\
\text { elliptical, around the proton } p \text {, since } \\
\text { Riemann geometry and positive gravity } \\
\text { are realized in }{ }^{L} S T\end{array}$ & $\begin{array}{l}\text { The atom and the antiprotium nucleus } \\
\text { include an antiproton, } \tilde{p} \text {, consisting of } \\
\text { three antiquarks: }(\tilde{u}, \tilde{u} \tilde{d}) \text { and positron, } \\
\text { that moves around antiproton - } \tilde{p} \text { along } \\
\text { hyperbolic } t \text {-soliton trajectories, since } \\
\text { Lobachevsky-Bolyai geometry and } \\
\text { negative gravity are realized in }{ }^{D} S T\end{array}$ \\
\hline $\begin{array}{l}\text { When discussing the motion of an } \\
\text { electron } e^{-} \text {(particle) around a proton } \\
p^{+} \text {(particle), it is known that an } \\
\text { electron } e^{-} \text {has an elementary point } \\
\text { electric negative charge } q^{-} \text {equal in } \\
\text { modulus to the positive point charge } \\
q^{+} \text {of a proton } p^{+}\end{array}$ & $\begin{array}{l}\text { When discussing the motion of a } \\
\text { positron } \tilde{e}^{+}(t \text {-soliton) around an } \\
\text { antiproton } \tilde{p}^{-}(t \text {-soliton), we assume } \\
\text { that the positron has an elementary } \\
\text { topological positive magnetic charge } \\
\tilde{\theta}^{+}, \text {equal in modulus to the negative } \\
\text { elementary topological magnetic charge } \\
\tilde{\theta}^{-} \text {of an antiproton } \tilde{p}^{-}\end{array}$ \\
\hline $\begin{array}{l}\text { - In }{ }^{L} S T \text {, particles of matter that have } \\
\text { opposite point electric charges attract }\end{array}$ & $\begin{array}{l}\text { In }{ }^{D} S T, t \text {-solitons of antimatter that } \\
\text { have opposite topological magnetic } \\
\text { charges repel }\end{array}$ \\
\hline
\end{tabular}

Thus, at any moment of time $T_{\text {synch }}$ in ${ }^{A} S T$ (see Figure 17), the attraction force ${ }^{L} F$ between negative electrically charged electron $e^{-}$and positive electrically charged proton $p^{+}$in ${ }^{L} S T$ is compensated by cumulative repulsion force ${ }^{D} F_{i j}$ between the positive magnetically charged positron $\tilde{e}^{+}$and the negative magnetically charged antiproton $\tilde{p}^{-}$in ${ }^{D} S T$. As a result, in ${ }^{A} S T$, between fundamentally entangled partners of electron $e^{-}$and positron $\tilde{e}^{+}$, and between the fundamentally entangled partners proton $p^{+}$and antiproton $\tilde{p}^{-}$, a dynamic 
equilibrium arises that prevents the electron $e^{-}$from falling onto the proton $p^{+}$in ${ }^{L} S T$, and positron $\tilde{e}^{+}$tearing away from the antiproton $\tilde{p}^{-}$in ${ }^{D} S T$.

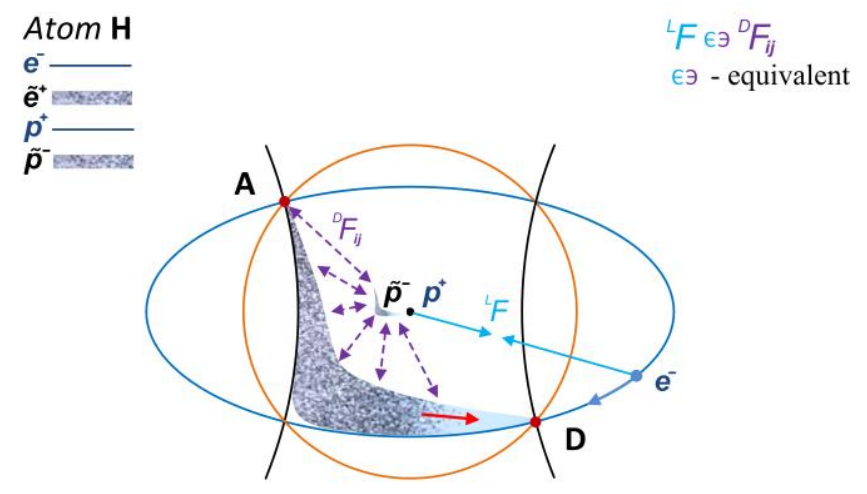

Figure 17. Self-consistent particle-soliton model of hydrogen atom (protium) in three space-times. At any moment $T_{\text {synch }}$ in ${ }^{A} S T$, electric force attraction ${ }^{L} F\left({ }^{L} t\right)$, acting between electron (particle) and proton (particle) in ${ }^{L} S T$, is equivalent to cumulative magnetic repulsive forces ${ }^{D} F_{i j}\left({ }^{D} t\right)$, acting between positron ( $t$ soliton) and antiproton ( $t$-soliton) in ${ }^{D} S T$. In general, nuclei (proton and antiproton) are not required to be located in the center and are placed there just for clarity. 


\section{Chapter 9 Fundamental interactions in space-times ${ }^{L} S T$ and ${ }^{D} S T$}

\begin{tabular}{|c|c|}
\hline Space-time ${ }^{L} S T$ & Space-time ${ }^{D} S T$ \\
\hline $\begin{array}{l}\text { - Gravitational interaction } \\
\text { Electromagnetic interaction: } \\
\text { - } \quad \text { Electric primary (elementary point } \\
\text { electric charges) } \\
\text { - } \quad \text { Magnetic secondary (elementary } \\
\text { magnetic charge is absent and is } \\
\text { present only in the form of a dipole) }\end{array}$ & $\begin{array}{l}\text { - Antigravitational interaction } \\
\text { - Positron-electric (strong) interaction: } \\
\text { - Positron (magnetic) primary } \\
\text { (elementary topological magnetic } \\
\text { charges) } \\
\text { - Electric secondary (elementary } \\
\text { electric charge is absent and is } \\
\text { present only in the form of a dipole) }\end{array}$ \\
\hline \multicolumn{2}{|c|}{ - Ideal exchange interaction } \\
\hline \multicolumn{2}{|c|}{ - Annihilation interaction } \\
\hline \multicolumn{2}{|c|}{ - Weak interaction } \\
\hline $\begin{array}{l}\text { In TSSCU, Brout-Englert-Higgs ( } B E H) \\
\text { about the origin of this field and of Higgs } \\
\text { as well as its distribution in space-tim } \\
\text { article, "TSSCU" Formalism. }\end{array}$ & $\begin{array}{l}\text { calar field is fundamental. The hypothesis } \\
\text { boson } H \text {, which has spin equal to } 0\left({ }_{i} S=0\right) \text {, } \\
{ }_{S}^{L} S T \text { and }{ }^{D} S T \text { will be detailed in the next }\end{array}$ \\
\hline
\end{tabular}

The electromagnetic and positron-electric (strong) interactions have already been discussed in Chapter 5, and the gravitational and antigravitational ones in Chapter 6. These 4 fundamental interactions are strictly divided according to their effect: gravitational and electromagnetic relate to ${ }^{L} S T$, and antigravitational and positron-electric (strong) relate to ${ }^{D} S T$.

Ideal exchange, annihilation, and weak interactions in TSSCU are interspatial, in which both space-times ${ }^{L} S T$ and ${ }^{D} S T$, to one degree or another, are directly involved. The ideal exchange interaction has already been discussed in Chapter 7. Despite the impressive successes of the modern theory of weak interaction $[113,114,115,116,117,118,119,120,121,122,123$, $124,125,126,127,128,129,130,131]$, from the standpoint of TSSCU it is incomplete. It makes sense to consider new approaches to this theory after understanding the role of Brout-Englert-Higgs $(B E H)$ scalar field and the Higgs boson $H$, which has spin equal to 0 $\left({ }_{i} S=0\right)$, which are directly involved in weak interaction.

When contemplating the annihilation interaction, that will be discussed in more detail in a separate article, "Specificities of the Gauge Theory and CPT Theorem in TSSCU", the following points that are directly related to annihilation should be considered.

As already discussed in section 6.8 of Chapter 6, anti-gravity acts in ${ }^{D} S T$, which breaks down antimatter. Decomposed antimatter, having negative mass and negative energy, takes on decentralized dynamic forms, referred before to as topological solitons. 
Hypothesis: $t$-solitons of antimatter, under certain conditions and circumstances, penetrate from heterogeneous and anisotropic space-time ${ }^{D} S T$ into space-time ${ }^{L} S T$, and are transformed into antiparticles by conforming to the action of gravity. Moreover, in accordance with the peculiarities of the CPT theorem in TSSCU (which will be discussed in more detail in the future publication "Specificities of the Gauge Theory and CPT Theorem in $\left.T S S C U^{\prime \prime}\right)$, in addition to the past spatial inversion ( $P$-inversion), time ${ }^{D} T$ is inverted by time ${ }^{L} T$ ( $T$ - inversion). In the case where $t$-solitons of antimatter has an elementary distributed magnetic charge, it is inverted into an electric charge opposite to the fundamentally entangled electric charge particles in ${ }^{L} S T$ ( $C$ - inversion). Thus, $t$-solitons of antimatter that are transformed into antiparticles, before meeting their fundamentally entangled partner and subsequent annihilation, are completely conforming to the laws acting in ${ }^{L} S T$. Actually, according to the author's interpretation, this precisely proves the experimental data and research done by various collaborations $[132,133,134,135,136,137,138,139,140,141$, $142,143,144,145,146,147,148,149,150,151]$. Nature tried to do everything to hide direct evidence of the reality of the existence of space-time ${ }^{D} S T$. 
Chapter 10 Fields and prevailing processes in space-time ${ }^{L} S T$ and ${ }^{D} S T$

\begin{tabular}{|l|l|}
\hline \multicolumn{1}{|c|}{ Space-time ${ }^{L} S T$} & \multicolumn{1}{c|}{ Space-time ${ }^{D} S T$} \\
\hline - $\quad$ Electric field (primary) & $\bullet \quad$ Positron field (magnetic primary) \\
$\begin{array}{l}\text { Form a quasi-electromagnetic field with a } \\
\text { predominant process of linear } \\
\text { homogenous expansion and emission of } \\
\text { electromagnetic waves (EMW) }\end{array}$ & $\begin{array}{l}\text { Form a quasi-positron electric field with a } \\
\text { predominant process of non-linear } \\
\text { heterogeneous compression and active } \\
\text { absorption (negative emission) of } \\
\text { positron-electric waves }(P E W \text { ) }\end{array}$ \\
\hline \multicolumn{2}{|c|}{ Space-time ${ }^{A} S T$} \\
\hline $\begin{array}{l}\text { As a result of the interaction of these two quasi-fields, while taking into account the } \\
\text { spin effect and ideal exchange interaction in }{ }^{A} S T \text { in } T_{\text {synch }} \text {, an electro-magnetic- } \\
\text { positron-electric self-consisted field is formed. }\end{array}$ \\
\hline
\end{tabular}




\section{Chapter 11 Principles of motion of material objects in space-time ${ }^{L} S T$ and ${ }^{D} S T$}

Considering the motion of a fundamentally entangled electron (particle) and a positron ( $t$-soliton) in a self-consistent atom in a stationary state

\begin{tabular}{|c|c|}
\hline Space-time ${ }^{L} S T$ & Space-time ${ }^{D} S T$ \\
\hline $\begin{array}{l}\text { Let an electron (particle) in moment of } \\
\text { time }{ }^{L} T \text { be at the localization point } A\end{array}$ & $\begin{array}{l}\text { Let a positron (particle) in moment of } \\
\text { time }{ }^{D} T \text { be at the localization point } A\end{array}$ \\
\hline
\end{tabular}

Spin ${ }_{t} S_{A}= \pm \frac{1}{2}$ at localization point $A$ is the "transparency pass window" in $T_{\text {synch }}$ in

${ }^{A} S T$, in which "the ideal exchange interaction" occurs between the electron particle and positron particle (particle because Euclidean geometry with a constant gravity ${ }^{A} G_{r}=1$ is realized in ${ }^{A} S T$ ), and the times ${ }^{L} T$ and ${ }^{D} T$ are being synchronized.

- Further, at the initial moment of time ${ }^{L} T$, positive gravity ${ }^{L} G_{r}$ acts on the electron (particle) and it insignificantly transforms from an ideal circumference to an oval

- Simultaneously, an electron using the impulse and the magnetic dipole (from the positron) received at the moment ${ }_{t} S_{A}=\frac{1}{2}$ moves clockwise along a trajectory different from circumference, tending to elliptical, to localization point $D$, in accordance with the least action principle
- Further, at the initial moment of time ${ }^{D} T$, negative gravity ${ }^{D} G_{r}$ acts on the positron (particle) and it is forced to transform into a $t$-soliton without dissipation by a hyperbolic wavelet (a sharp increase in amplitude with compensatory formation of dispersion)

- Simultaneously, a positron using the impulse and electric dipole (from the electron), received at the moment ${ }_{t} S_{A}=-\frac{1}{2}$, moves counterclockwise along the hyperbolic t-soliton trajectories to the localization point $D$

Spin ${ }_{t} S_{D}= \pm \frac{1}{2}$ at localization point $D$ is the "transparency pass window" in $T_{\text {synch }}$ in ${ }^{A} S T$, in which "the ideal exchange interaction" occurs between the electron particle and positron particle (particle, because Euclidean geometry with a constant gravity ${ }^{A} G_{r}=1$ is realized in ${ }^{A} S T$ ) and the times ${ }^{L} T$ and ${ }^{D} T$ are synchronized. 
- Further, at the initial moment of time ${ }^{L} T$, positive gravity ${ }^{L} G_{r}$ acts on electron (particle) and it insignificantly transforms from an ideal circumference to an oval

- Simultaneously, an electron using the impulse and the magnetic dipole (from the positron) received at the moment ${ }_{t} S_{D}=\frac{1}{2}$ moves clockwise along a trajectory different from circumference and tending to elliptical to localization point $A$, in accordance with the principle of least action
- Further, at the initial moment of time ${ }^{D} T$, negative gravity ${ }^{D} G_{r}$ acts on positron (particle) and it is forced to transform into a $t$-soliton without dissipation by a hyperbolic wavelet (a sharp increase in amplitude with compensatory formation of dispersion)

- Simultaneously, a positron using the impulse and electric dipole (from the electron) received at the moment ${ }_{t} S_{D}=-\frac{1}{2}$ moves counterclockwise along the hyperbolic t-soliton trajectories to the localization point $A$

Despite the chaotic properties of ${ }^{D} S T$, a positron ( $t$-soliton), being fundamentally entangled to an electron (particle), successfully fulfills with its task: to start and end its movement at localization points $A$ and $D$ (or $B$ and $C$ ). In addition to the positron, other anti-matter $t$ solitons, including dark photons being fundamentally entangled to their partners in ${ }^{L} S T$, also move in concert as a single well-controlled organism. It is necessary to take into consideration one more important circumstance: the dependence on the geometry realized in space-time for $t$-solitons of anti-matter in ${ }^{D} S T$ is much higher than for matter particles in ${ }^{L} S T$. Given the many factors described in this article, assuming that due to the motion of the positron $t$-soliton (and other fundamentally-entangled $t$-solitons of antimatter) in ${ }^{D} S T$, some new principle must be realized, equivalent to the principle of least action for electron (and other particles) in ${ }^{L} S T$. They are referred to as principle of the greatest action, because in space-time ${ }^{D} S T$ having a fractional dimension, an inverse relationship is realized (see section 3.7). The packets of $t$-solitons elements of antimatter, that are constantly decaying and moving to the point of their next localization, are assumed to act in accordance with this new proposed principle. Taking into account the idea of Feynman and Hibbs [152], that the "positron" (based on the author's interpretation, taking into account the positions suggested in this article, but Feynman and Hibbs obviously talked about the electron) goes along all possible trajectories to the point of its localization, then this is additional confirmation of the correctness of the new principle. Therefore, it should be noted that both principles, the least action in ${ }^{L} S T$ and the greatest action in ${ }^{D} S T$, must be considered inextricably combined with each other. In fact, each one of them serves as the main evidence of "correctness" of each other.

The nature of $t$-solitons is primary in ${ }^{D} S T$, therefore, both elementary $t$-solitons and groups (ensembles) of interacting $t$-solitons are realized in it. In ${ }^{L} S T$, the nature of $t$-solitons is secondary, therefore only secondary groups (ensembles) of interacting $t$-solitons are 
realized in it. These and similar processes can be observed in nature, even at the macro level. For example, the murmuration of a flock of birds, or the movement of a swarm of bees or schools of fish [153], which all await their mathematical solution.

Further development of the theory of motion of $t$-solitons of antimatter in ${ }^{D} S T$ is possible in two ways. First, despite the existing significant achievements, for example, the works described in $[154,155,156,157,158,159]$, it is necessary to adapt existing nonlinear differential equations having soliton solutions to realities of ${ }^{D} S T$. Second, new equations for ${ }^{D} S T$ are needed, that take into account the new knowledge and the whole process, from the beginning of $t$-soliton (hyperbolic wavelet with formation of a tree crown-network) formation to its localization (in a point of tree trunk), in accordance with the principle of the greatest action. Taking into account the conclusions obtained in the section 3.9 of the Chapter 3 regarding the nature of complex numbers, a promising approach to solving the problem along the first path is partially implemented in the work of Fokas [160].

Conclusion 1: The electron at localization points $A$ and $D$ (or $B$ and $C$ ) in ${ }^{A} S T$, as well as in time ${ }^{L} T$, invariably represents a particle, and moves in ${ }^{L} S T$ according to the principle of least action. The positron at localization points $A$ and $D$ (or $B$ and $C$ ) in ${ }^{A} S T$ represents a particle (since Euclidean geometry $(\mathcal{E})$ with constant gravity ${ }^{A} G_{r}=1$ is realized there), and in time ${ }^{D} T$, represents a $t$-soliton ( $s$-cloud), and moves in ${ }^{D} S T$ along hyperbolic $t$-soliton trajectories, in accordance with the principle of the greatest action.

Conclusion 2: The Schrödinger equation describes the state of a system of fundamentally entangled electron particles in ${ }^{L} S T$ and $t$-soliton ( $s$-clouds) of positron in ${ }^{D} S T$. This surprising conclusion will be more convincing if we take into account that in time-depended Schrödinger equation, time $\left({ }^{L} t\right)$ appears as a first-order derivative, and spatial coordinates $\left({ }^{D} S T\right.$ or projections of ${ }^{D} S T$ into $\left.{ }^{A} S T\right)$ are expressed as second-order derivatives. Thus, in the Schrödinger equation, space and time are heterogeneous, that is, they belong to different space-times.

At the same time, a complex-valued wave function is a mathematical tool that provides some statistical and other information about the motion of a $t$-soliton ( $s$-cloud) of a positron along hyperbolic $t$-soliton orbits in ${ }^{D} S T$. When conducting quantum measurements, a researcher's tool (consisting of both particles of matter and $t$-solitons of antimatter) interacts with an element (or several elements) of a positron $t$-soliton ( $s$-cloud) packet in ${ }^{D} S T$. Leading to the natural instantaneous reaction (in $T_{\text {synch }}$ ) of a system of fundamentally entangled electron and positron. As a consequence of this reaction, the wave function, up to this point describing the state of the system, collapses.

In the case of an non-stationary (highly excited) state of an atom and various resonance phenomena, it is impossible to exclude a primary hyperbolic positron wavelet with the appearance of "very large" amplitude, and, accordingly, a nonzero probability of finding positron $t$-soliton elements in any region of space-time ${ }^{D} S T$. 
Conclusion 3: The Dirac equation can be interpreted as describing a system of relativistic fundamentally entangled electron particles in ${ }^{L} S T$ with positive mass and positive energy, with two localization points with spin ${ }_{t} S= \pm \frac{1}{2}$ in ${ }^{A} S T$ and positron $t$-soliton in ${ }^{D} S T$ with negative mass and negative energy, with two localization points with spin ${ }_{t} S= \pm \frac{1}{2}$ in ${ }^{A} S T$.

Conclusion 4: The Heisenberg uncertainty principle only applies if the researcher limits himself to the framework of space-time ${ }^{L} S T$. In this case, when finding the exact coordinates of the electron in ${ }^{L} S T$, its future impulse to the moment of next localization point is in spacetime ${ }^{D} S T$, and information about it (impulse) is uncertain. If the researcher goes beyond ${ }^{L} S T$ and takes measurements in both space-times ${ }^{L} S T$ and ${ }^{D} S T$, synchronously in $T_{\text {synch }}$, the state of the electron in ${ }^{L} S T$, and the state of the positron in ${ }^{D} S T$, using all 6 dimensions (4 spatial and 2 temporal) for each space-time, then he can get any required information with absolute accuracy.

Conclusion 5: A rigorous mathematical definition of $t$-soliton (charged and / or neutral) as a form of antimatter in space-time ${ }^{D} S T$ is the priority task. The exact mathematical formalization of the principle of the greatest action for the motion of $t$-solitons of antimatter in space-time ${ }^{D} S T$ under conditions of heterogeneity and anisotropy, space and time ${ }^{D} T$, as well as finding the equations of motion, is an extremely important task. Thus, the ultimate goal is to find a system of two-component self-consistent equations of motion in $T_{\text {synch }}$ : an electron (particle) in ${ }^{L} S T$, in accordance with the principle of least action, and a positron $(t-$ soliton) in ${ }^{D} S T$, in accordance with the principle of the greatest action using all 6 dimensions for each space-time. 


\section{Chapter 12 Conclusion}

1. Universe is one and eternal. The Universe includes primary (maternal) Absolute spacetime ${ }^{A} S T$ and Controlling Superimaginary Unit ${ }_{S} I$, which periodically initiates occurrence of $B B$ with the subsequent inevitable $C r$.

2. The approach for determining the dimension of the Universe and its architecture can be as follows: based on the hypothesis and results presented in this article, one can assume that in the period from $C r$ to $B B$, primary (maternal) Absolute space-time ${ }^{A} S T$ is in an uncertain state, but Controlling Superimaginary Unit ${ }_{S} I$ does not interact with ${ }^{A} S T$ and is in a detached state.

In the moment of $B B$ to $C r$, primary (maternal) Absolute space-time ${ }^{A} S T$ (zero $(\Omega=0$ ) and infinity $(\Omega=\infty)$ curvatures simultaneously) interacts with imaginary parabolic unit $I$ forming generalized Paracomplex space-time ${ }_{I}^{A} S T$, consisting of 6 Paracomplex dimensions. In the case of partial bundle of this generalized Paracomplex Absolute spacetime ${ }_{I}^{A} S T$ they (dimensions) triple, forming 18 dimensions:

- 3 spatial coordinates of ${ }^{A} S T$

- 1 time ${ }^{A} T$

- 1 imaginary parabolic spatial unit $I$

- 1 imaginary parabolic time ${ }_{I} T$

- 3 spatial coordinates of ${ }^{L} S T$

- 1 time ${ }^{L} T$

- 1 imaginary elliptic spatial unit $i$ in ${ }^{L} S T$

- 1 imaginary spatial time ${ }_{i} t$ in ${ }^{L} S T$

- 3 spatial coordinates of ${ }^{D} S T$

- 1 dark time ${ }^{D} T$

- 1 dark imaginary hyperbolic spatial unit $\tilde{\imath}$ in ${ }^{D} S T$

- 1 dark imaginary hyperbolic time ${ }_{i} t$ in ${ }^{D} S T$

Thus, taking into account the author's prioritized hypothesis of the partial bundle of the generalized Paracomplex Absolute space-time ${ }_{I}^{A} S T$, at the present stage in the Universe, 12 rational and 6 imaginary dimensions are realized or:

- 4 spatial and 2 temporary Paracomplex dimensions in ${ }_{I}^{A} S T$

- 4 spatial and 2 temporary complex dimensions in ${ }_{i}^{L} S T$

- 4 spatial and 2 temporary dark complex dimensions in ${ }_{i}^{D} S T$

3. At the present stage in the Universe, relative to the generalized Paracomplex Absolute space-time ${ }_{I}^{A} S T$, space-time symmetry is realized. At the moment $B B$, two mutually transparent space-times, ${ }^{L} S T$ and ${ }^{D} S T$, were born (formed) synchronously in $T_{\text {synch }}$. Tissue of space-time ${ }^{L} S T$ homogenously expands (relative to the stationary tissue of ${ }^{A} S T$ ) by heterogeneously compressing the tissue of space-time ${ }^{D} S T$. The opposite is also 
true, as the tissue of space-time ${ }^{D} S T$ is heterogeneously compressed (relative to the stationary tissue of ${ }^{A} S T$ ) due to the homogenous expansion of the tissue of space-time ${ }^{L} S T$.

4. The proposed bundle algorithms for Absolute space-time ${ }^{A} S T$ with Euclidean geometry $(\varepsilon)$, in the form of equations 5-23, are preliminary and incomplete and need to be expanded by:

- Inclusion of three imaginary units: parabolic $I$, elliptic $i$, hyperbolic $\tilde{i}$, each of which has its own space-time

- Determining and including an expansion quant of space-time ${ }^{L} S T$, self-consistent with dark compression quants of space-time ${ }^{D} S T$

- Studying the nature and properties of synchronization time $T_{\text {synch }}$

5. The fate of those who formed synchronously in $T_{\text {synch }}$ in the generalized Paracomplex Absolute space-time ${ }_{I}^{A} S T$, at the moment $B B$, of two space-times ${ }^{L} S T$ and ${ }^{D} S T$, is predetermined and in the future they will disappear, also synchronously, at the moment $\mathrm{Cr}$ in $T_{\text {synch }}$.

6. Baryonic asymmetry in the Universe is absent. All the born matter develops since from the moment $B B$, in the space-time we observe, of positive curvature ${ }^{L} S T$, and all the born antimatter develops, in the not observed by us, space-time of negative curvature ${ }^{D} S T$. That part of antimatter, which for some reasons (will be considered in one of the subsequent articles "Specificities of the Gauge Theory and the CPT Theorem in TSSCU"), penetrates into space-time ${ }^{L} S T$ - annihilates with matter while maintaining parity.

7. Antimatter with a negative density of matter and a negative density of energy, formed at the moment $B B$ and developing in space-time ${ }^{D} S T$, can also be called dark matter (or hidden matter) possessing dark energy (hidden energy). In accordance with partial bundle of ${ }_{I}^{A} S T$ the main density in the Universe consists of primary controlling matter ${ }^{A} M$ with primary controlling energy ${ }^{A} E$.

8. The birth at the moment $B B$ of two mutually transparent and, at the same time selfconsistent space-times ${ }^{L} S T$ and ${ }^{D} S T$ with their own times ${ }^{L} T$ and ${ }^{D} T$, also forces us to introduce the concept of time synchronization $T_{\text {synch }}$. One of the most important properties of $T_{\text {synch }}$ is that the formation of paired objects in space-times ${ }^{L} S T$ and ${ }^{D} S T$ synchronous in $T_{\text {synch }}$ gives these objects the property of fundamental entanglement $\bar{æ}$.

9. Realization of the "effect of self-consistency" in the Universe became possible, due to the new way of introducing generalized complex numbers. Each imaginary unit: parabolic $I$, elliptic $i$ and hyperbolic $\tilde{l}$ is in strict accordance with the curvature sign of its space-time. Specifically, the generalized complex numbers that develop the theories of functions of 
complex variables, play the main role in providing inter space-time communication (the subsequent article "TSSCU Formalism" will be dedicated to it).

10. The nature of mass, particles of matter with mass ${ }^{L} m$ and $t$-solitons of antimatter with mass ${ }^{D} m$ are born in pairs synchronously in $T_{\text {synch }}$ from the cells of tissues of spacetimes ${ }^{L} S T$ and ${ }^{D} S T$, accordingly. In this case, particles with mass ${ }^{L} m$ and $t$-solitons with mass ${ }^{D} m$ will have the property of fundamental entanglement. An exception to this rule is the mass of the Higgs boson $H$, which spin equal to $0\left({ }_{i} S=0\right)$, which plays its fundamental role in $T S S C U$.

11. Understanding the three space-time nature of the spin ${ }_{t} S$ (transparency pass window in ${ }^{A} S T$ ) allows us not only to formalize the "ideal exchange interaction" for fermions and bosons, but also to make the following accurate, non-alternative predictions:

- Particles that are antiparticles of themselves do not exist;

- Massless neutral boson photon $\gamma$ in ${ }^{L} S T$, with ${ }_{t} S= \pm 1$ must have a partner of a massless neutral dark photon $\tilde{\gamma}$ in ${ }^{D} S T$, as well as with $\operatorname{spin}{ }_{t} S= \pm 1$;

- Massive boson ${ }^{0} Z$ in ${ }^{L} S T$ with $\operatorname{spin}{ }_{t} S= \pm 1$ must have a partner of a neutral massive boson from antimatter ${ }^{0} \tilde{Z}$ in ${ }^{D} S T$, as well as with $\operatorname{spin}{ }_{t} S= \pm 1$.

12. The principles of least action for particles of matter in ${ }^{L} S T$ and the greatest action for $t$ solitons of antimatter in ${ }^{D} S T$ and their fundamental entanglement and self-consistency in motion open up wide possibilities to construct accurate mathematical models of motion in space-times ${ }^{L} S T$ and ${ }^{D} S T$.

13. The concept of fundamental entanglement, the principles of least and the greatest actions, "ideal exchange interaction", $t$-solitons as a form of antimatter in ${ }^{D} S T$, as well as the hypothesis that positron in ${ }^{D} S T$ has an elementary topological magnetic charge, lead to the final stage of constructing the theory of a self-consistent particle-soliton atom.

14. The strong nuclear interaction, applied in physics nowadays, is a positron-electric interaction in space-time ${ }^{D} S T$ in $T S S C U$. In this case, the positron and antiproton, as well as other charged $t$-solitons, in ${ }^{D} S T$ have an elementary topological magnetic charge $\tilde{\theta}$, the quantitative value of which remains to be determined. In addition, space-time ${ }^{D} S T$ must have its own dark action quants, ${ }^{D} h$ and ${ }^{D} \hbar_{1-n}$, equivalent to Planck constant ${ }^{L} h$ and reduced Planck constant ${ }^{L} \hbar$. The determination of the values of an elementary topological magnetic charge $\tilde{\theta}$, as well as dark action quants (active absorption or attraction) ${ }^{D} h$ and ${ }^{D} \hbar_{1-n}$ will, in the foreseeable future, create a complete theory of strong interaction, in the form of a system of equations for ${ }^{D} S T$, equivalent to the modified Maxwell equations for ${ }^{L} S T$.

15. To solve the problem of gravity quantization, accounting for the three space-time model of the structure of the Universe, a question needs to be proposed in a new way: it is necessary to define an expansion quant (in a pair: distance-radius of curvature ${ }^{L_{S}} S$ and 
curvature ${ }^{L} \omega$ ) in ${ }^{L} S T$, as well as dark compression quants (in pair: distance-radius of curvature ${ }^{D} S$ and curvature ${ }^{D} \omega$ ) in ${ }^{D} S T$. To do so, it is necessary to formulate and find solutions to the self-consistent system of equations describing the action of antigravity (topological rotational stabilization mechanism) in ${ }^{D} S T$.

In addition, despite the dynamic chaotic properties of space-time ${ }^{D} S T$, it is necessary to try and find approaches to writing equations for a universal anti-gravitation law(s) in ${ }^{D} S T$, that is equivalent to law of universal gravitation in ${ }^{L} S T$.

16. The hypothesis that thermonuclear reactions are the main source of energy in the Universe is not complete.

The manifold of existing material and antimaterial worlds in the three space-time Universe can be explained by the mutual transparency of space-times ${ }^{L} S T$ and ${ }^{D} S T$ and their fundamental entanglement and mutual antagonism. 


\section{Used terms, symbols and abbreviations}

${ }^{A} S T$ - primary (maternal) Absolute space-time

$s^{I}$ - Controlling Super-imaginary Unit

I - imaginary parabolic unit

${ }_{I}^{A} T$ - imaginary parabolic time

${ }_{I}^{A} S T$ - generalized Paracomplex Absolute space-time

${ }^{A} M$ - primary matter in ${ }^{A} S T$ with imaginary parabolic unit $I$

${ }^{A} E$ - primary energy in ${ }^{A} S T$ with imaginary parabolic unit $I$

${ }^{A} I$ - primary information in ${ }^{A} S T$ with imaginary parabolic unit $I$

${ }^{A} m$ - mass of a particle in ${ }^{A} S T$

${ }^{A} S$ - distance in ${ }^{A} S T$

${ }^{A} \Omega$ - curvature in ${ }^{A} S T$

${ }^{A} T$ - time in ${ }^{A} S T$

${ }^{A} V$ - velocity in ${ }^{A} S T$

${ }^{A} G_{r}$ - gravity in ${ }^{A} S T$

${ }^{A} K$ - temperature in ${ }^{A} S T$

$B B$ - Big Bang

$\mathrm{Cr}$ - Crunch

${ }^{S T} U$ - interdimensional potential

$T_{\text {synch }}$ - synchronization time

${ }_{t} S$ - spin

$(\mathcal{E})$ - Euclidean geometry

$\bar{\not}$ - fundamental entanglement between space-times ${ }^{L} S T$ and ${ }^{D} S T$, as well as particles in ${ }^{L} S T$ and its partners $t$-solitons in ${ }^{D} S T$

${ }^{L} S T$ - Light (Real) space-time

$i$ - imaginary elliptic unit

${ }_{i} t$ - imaginary elliptic time in ${ }^{L} S T$

${ }_{i}^{L} S T$ - Elliptic complex space-time 
${ }^{L} M$ - matter (with positive density) in ${ }^{L} S T$

${ }^{L} E$ - energy (with positive density) in ${ }^{L} S T$

${ }^{L} I$ - stochastic information in ${ }^{L} S T$

${ }^{L} m$ - mass of a particle in ${ }^{L} S T$

${ }^{L} S$ - distance in ${ }^{L} S T$

${ }^{L} \omega$ - curvature in ${ }^{L} S T$

${ }^{L} T$ - time in ${ }^{L} S T$

${ }^{L} v$ - velocity in ${ }^{L} S T$

${ }^{L} G_{r}$ - gravity in ${ }^{L} S T$

${ }^{L} K$ - temperature in ${ }^{L} S T$

$c$ - speed of light in ${ }^{L} S T$

${ }^{L} \gamma$ or $\gamma$ - photon in ${ }^{L} S T$

$e^{-}$- electron

$\dot{e}^{+}$- positron in ${ }^{L} S T$

$q$-elementary point electric charge in ${ }^{L} S T$

$\theta^{+-}$- dipole magnetic charges in ${ }^{L} S T$

${ }^{l} h$ - Planck constant in ${ }^{L} S T$

${ }^{l} \hbar$ - reduced Planck constant in ${ }^{L} S T$

$l_{p}$ - Planck length in ${ }^{L} S T$

${ }^{L} G$ - gravitational constant in ${ }^{L} S T$

${ }^{L} H_{b}$ - Hubble's dynamic parameter in ${ }^{L} S T$

$G R$ - general theory of relativity

LQG - Loop Quantum Gravity

$(\mathcal{R})$ - Riemann geometry

${ }^{D} S T$ - dark (Hidden) space-time

$\tilde{\imath}$ - dark imaginary hyperbolic unit

${ }_{i} t$ - dark imaginary hyperbolic time in ${ }^{D} S T$

${ }_{i}^{D} S T$ - Hyperbolic dark complex space-time 
${ }^{D} M$ - dark matter (antimatter with negative density) in ${ }^{D} S T$

${ }^{D} E$ - dark energy (negative energy density) in ${ }^{D} S T$

${ }^{D} I$ - fractal information in ${ }^{D} S T$

${ }^{D} m$ - mass formation in ${ }^{D} S T$

${ }^{D} S$ - distance in ${ }^{D} S T$

${ }^{D} \omega$ - curvature in ${ }^{D} S T$

${ }^{D} T$ - time in ${ }^{D} S T$

${ }^{D} v$ - velocity in ${ }^{D} S T$

${ }^{D} G_{r}$ - antigravity in ${ }^{D} S T$

${ }^{D} K$ - temperature in ${ }^{D} S T$

${ }^{D} \gamma$ or $\tilde{\gamma}$ - dark photon in ${ }^{D} S T$ (gluon - g, magnon, are also ${ }^{D} \gamma$ )

$\tilde{c}$ - speed of dark light in ${ }^{D} S T$ for algebra with dominant minus

$\frac{1}{\tilde{c}}$ - speed of dark light in ${ }^{D} S T$ for algebra with an absolute dominant minus

$\widetilde{e}^{+}$- positron in ${ }^{D} S T$

$\tilde{\theta}$ - elementary topological magnetic charge in ${ }^{D} S T$

$\tilde{q}^{-+}$- dipole topological electric charges in ${ }^{D} S T$

${ }^{d} h$ - fluctuation parameter of action quant in ${ }^{D} S T$ equivalent to Planck constant ${ }^{1} \mathrm{~h}$ in ${ }^{L} S T$

${ }^{d} \hbar_{1-n}$-fluctuation parameter of action quant in ${ }^{D} S T$ equivalent to reduced Planck constant ${ }^{\mathrm{l}} \hbar$ in ${ }^{L} S T$

${ }^{D} G_{1-n}$ - antigravitational fluctuation parameter in ${ }^{D} S T$

${ }^{D} H_{b}$ - dynamic fluctuation parameter in ${ }^{D} S T$ equivalent to Hubble parameter in ${ }^{L} S T$

$S N T$ - String Network Theory

$(\mathcal{L B})$ - Lobachevsky-Bolyai geometry

$B E H$ - scalar field of Braut-Engler-Higgs

$H$ - Higgs boson

$S T$ - String Theory

CST - Controlling String Theory 


\section{References}

[1] A. Einstein, Die Feldgleichungen der Gravitation, Sitzungsberichte der Preuss. Akad. der Wissenschaften, Berlin, (1915), P. 844-847.

[2] D. Hilbert «Die Grundlagen der Physic», Nachrichten Kon. Gesellshaft Wiss. Gottingen, Math. - Phys. Klasse, Heft 3, (1915), P. 395-407.

[3] A. Einstein, " Cosmological Considerations in the General Theory of Relativity " Sitzungsberichte der Preuss. Akad. der Wissenschaften, Berlin, (1917), P. 142-152.

[4] W. de Sitter, On Einstein's Theory of Gravitation and its Astronomical Consequences, Monthly Notices of the Royal Astronomical Society, Vol. 76, Iss. 9, (1916), P. 699-728.

[5] Friedmann, A: «Über die Krümmung des Raumes», Zeitschrift für Physik, Vol. 10, (1922), P. 377-386.

[6] Friedmann, A: «Über die Möglichkeit einer Welt mit konstanter negativer Krümmung des Raumes», Zeitschrift für Physik, Vol. 21, (1924), P. 326-332.

[7] Kaluza T. "Zum Unitätsproblem der Physic", Sitzungsberichte der Preuss. Akad. der Wissenschaften, Berlin, Math. - Phys., (1921), P. 966-972.

[8] Klein O., Quantentheorie und fünfdimensionale Relativitätstheorie, Zeitschrift für Physik, Vol. 37, (1926), P. 895-906.

[9] Lemaître, G. "A Homogeneous Universe of Constant Mass and Growing Radius, Accounting for the Radial Velocity of the Extragalactic Nebulae", Annales de la Société Scientifique de Bruxelles 47, (1927), P. 49-59.

[10] Vesto M. Slipher, Nebulae., Proc. Am. Philos. Soc., Vol. 56, (1917), P. 403-409.

[11] Howard P. Robertson, "On Relativistic Cosmology", Philos. Mag. and Journal of Science, Vol. 5, (1928), P. 835-848.

[12] Edwin P. Hubble, A Relation between distance and radial velocity among extra-galactic nebulae, Proc. Nat. Acad. Sci., 15 (3), (1929), P. 168-173.

[13] Ralph A. Alpher and Robert Herman, Evolution of the Universe, Nature, 162, (1948), P. 774-775.

[14] Dicke R. H., Peebles P. J. E., Roll P. G., Wilkinson D. T., Cosmic Black-Body Radiation., The Astrophysical Journal, Vol. 142, (1965), P. 414-419.

[15] Penzias A. A., Wilson R. W., A Measurement of Excess Antenna Temperature at 4080 Mc/s., The Astrophysical Journal, Vol. 142, (1965), P. 419-421. 
[16] F. Hoyle, A New Model for the Expanding Universe, Monthly Notices of the Royal Astronomical Society, Vol. 108, Iss. 5, (1948), P. 372-382.

[17] H. Bondi, T. Gold, The Steady-State Theory of the Expanding Universe, Monthly Notices of the Royal Astronomical Society, Vol. 108, Iss. 3, (1948), P. 252-270.

[18] Gustav Naan, "Symmetrical Universe”, Publications of Tartu Astronomical observatory, Vol. XXXIV, (1964), P. 423-471.

[19] W. Tiller, "The Positive and Negative Space-Time Frames as Conjugate Systems", (Stanford, Preprint (1975), in Future Science, ed. White and Krippner (Garden City, NY: Doubleday \& Co., Inc.), (1977), P. 257-279.

[20] John A. Wheeler, Genesis and Observership, Foundational Problems in the Special Sciences., Ed. by R. E. Butt, J. Hintikka, Dordrecht: D. Reidel, (1977), P. 3-33.

[21] Barrow J. D., Tipler F. J., The Anthropic Cosmological Principle., Clarendon, Oxford University Press, (1986).

[22] David Bohm, "Hidden Variables and the Implicate Order", Zygon 20 (2), (1985), P. 111-124.

[23] Alain Connes, Noncommutative Geometry, (InterEditions Paris (1990)) San Diego: Academic Press, (1994), 654 p.

[24] Strukov I. A., Brukhanov A. A., Skulachev D. P. Sazhin M. V., Anisotropy of the microwave background radiation, Pis'ma v Astronomicheskii Zhurnal (Astronomy Letters), Vol. 18, N. 5, (1992), P. 387-395.

[25] George F. Smoot, C.L. Bennett, A. Kogut, E.L. Wright, J. Aymon, N.W. Boggess, E.S. Cheng, G. De Amici, S. Gulkis, M.G. Hauser, et al. "Structure in the COBE differential microwave radiometer first year maps”, Ap. J. Lett., 396, (1992), L1.

[26] Adam G. Riess, Alexei V. Filippenko, Peter Challis, Alejandro Clocchiatti, Alan Diercks, Peter M. Garnavich, Ron L. Gilliland, Craig J. Hogan, Saurabh Jha, Robert P. Kirshner, et al., Observational Evidence from Supernovae for an Accelerating Universe and Cosmological Constant, Astronomical Journal, Vol. 116, (1998), P. 1009-1038.

[27] S. Perlmutter, G. Aldering, G. Goldhaber, R.A. Knop, P. Nugent, P.G. Castro, S. Deustua, S. Fabbro, A. Goobar, D.E. Groom, et al., Measurements of Omega and Lambda from 42 HighRedshift Supernovae, Astrophysical Journal, Vol. 517, (1999), P. 565-586.

[28] Yu. S. Vladimirov, The Nature of Space and Time: An Anthology of Ideas, Moscow: Lenand, (2015), 400 p.

[29] Schwartz M. D., Quantum Field Theory and Standard Model, Cambridge University Press, (2013), 952 p. 
[30] V. L. Ginzburg, "What problems of physics and astrophysics seem now to be especially important and interesting?", UFN, Vol. 169, N. 4, (1999), P. 419-441.

[31] I. F. Ginzburg, Unsolved problems in fundamental physics, UFN, Vol. 179, N. 5, (2009), P. 525-529.

[32] John C. Baez. Open Questions in Physics, (2012).

http://math.ucr.edu/home/baez/physics

[33] S. Weinberg, Lectures in Particles and Field Theory, ed. S. Deser and K. Ford, Phys. Lett. 9, (1964), p. 357; Phys. Rev. 8138, (1965), p. 988.

[34] A. D. Sakharov, Violation of CP Invariance, C asymmetry, and baryon asymmetry of the universe, JETP Lett., Vol. 5, (1967), P. 32-35.

[35] Tolman R. C. On the Problem of the Entropy of the Universe as a Whole., Phys. Rev., 37, (1931), P. 1639-1660.

[36] Tolman R. C. On the Theoretical Requirements for a Periodic Behaviour of the Universe., Phys. Rev., 38, (1931), P. 1758-1771.

[37] Starobinsky A. A. Spectrum of relict gravitational radiation and the early state of Universe. JETP Lett., Vol. 30, (1979), P. 682-685.

[38] Starobinsky A. A. A new type of isotropic cosmological model without singularity. Phys. Lett. B, Vol. 91, (1980), P. 99-102.

[39] Guth A. H. Inflationary Universe: A possible solution to the horizon and flatness problems. Phys. Rev. D, Vol. 23, (1981), P. 347-356.

[40] Mukhanov V. F., Chibisov G. V. Quantum Fluctuation and "Nonsingular Universe". JETP Lett., Vol. 33, (1981), P. 532-535.

[41] Linde A. D. A New Inflationary Universe Scenario: A Possible Solutions of the Horizon, Flatness, Homogeneity, Isotropy and Primordial Monopole Problems. Phys. Lett. B, Vol. 108, (1982), P. 389-393.

[42] Albrecht A., Steinhardt P. J., Cosmology for Grand Unified Theories with Radiatively Induced Symmetry Breaking. Phys. Rev. Lett., Vol. 48, (1982), P. 1220-1223.

[43] A.D. Linde, "Inflationary Cosmology", Lecture Notes in Physics, 738, (2008), P. 1-54.

[44] A. Ijjas, P. J. Steinhardt, A. Loeb, "Inflationary paradigm in trouble after Planck 2013", Phys. Lett. B. 723, (2013), P. 261-266.

[45] Guth A. H., Kaiser, D. I. and Nomura, Y., "Inflationary Paradigm after Planck 2013," Physics Letters B. 733, (2014), P. 112-119.

[46] A. Linde, “Inflationary Cosmology after Planck 2013”, arXiv:1402.0526, (2014), P. 79. 
[47] A. Ijjas, P. J. Steinhardt, A. Loeb, “Inflationary schism”, Phys. Lett. B. 736, (2014), P. 142-146.

[48] A. D. Sakharov, "The initial stage of an expanding Universe and the appearance of a non-uniform distribution of Matter", JETP Letter, Vol. 49, (1965), P. 345-358.

[49] Ia. B. Zeldovich, "The "hot" model of the Universe", UFN, Vol. 89, (1966), P. 647-668.

[50] Ia. B. Zeldovich, I. D. Novikov, «Structure and evolution of the Universe", Moscow: Izdatel'stvo Nauka, (1975), 736 p., P. 657-663.

[51] A. Einstein, Zum kosmologischen Problem der allgemeinen Relativitätstheorie, Sitzungsberichte der Preuss. Akad. der Wissenschaften, Berlin, (1931), P. 235-237.

[52] A. D. Sakharov, "Cosmological Models of the Universe with Rotation of Time's Arrow", JETP Letter, Vol. 79, Iss. 3(9), (1980), P. 689-693.

[53] A. D. Sakharov, «Multi-sheeted models of the universe», JETP Letter, Vol. 83, (1982), P. 1233-1240.

[54] A. D. Sakharov, "Cosmological transitions with change of the sign of metrics", JETP Letter, Vol. 87, (1984), P. 375-383.

[55] P. J. Steinhardt, Neil Turok, A Cyclic Model of the Universe, Science, V. 296, (2002), P. 1436-1439.

[56] Lauris Baum, Paul H. Frampton, Turnaround in Cyclic Cosmology, Phys. Rev. Lett., Vol. 98, N. 7, (2007), 071301.

[57] Latham Boyle, Kieran Finn, Neil Turok «CPT-Symmetric Universe», Phys. Rev. Lett., 121(25), (2018), 251301(5).

[58] J. S. Farnes «A unifying theory of dark energy and dark matter: Negative masses and matter creations within a modified $\Lambda$ CDM framework», A\&A, 620, A92, (2018).

[59] A. Einstein, B. Podolsky, N. Rosen, «Can Quantum-Mechanical Description of Physical Reality Be Considered Complete? », Phys. Rev., Vol. 47, Iss. 10, (1935), P. 777-780;

[60] Feynman R. The theory of positrons. Phys. Rev., Vol. 76, Iss. 6, (1949), P. 749-759.

[61] H. Poincaré, "The Measure of Time", (Revue de métaphysique et de morale, 6: 1-13, 1898), The Foundations of Science, N.-Y.: Science Press, (1913), P. 222 - 234.

[62] H. A. Lorentz "Electromagnetic phenomena in a system moving with any velocity smaller than that of light", KNAW (Amsterdam), Proceedings, 6, (1904), P. 809-813. [63] A. Einstein, "Zur Elektrodynamic der bewegter Körper", Ann. Physik, 17, (1905), P. 891-921. 
[64] H. Poincaré "Sur la dynamique de l'électron", Rendiconti del Circolo matematico di Palermo, 21, (1906), P. 129-176.

[65] A. Einstein, "Einfluss der Schwerkraft auf die Ausbreitung des Lichtes", Ann. Physik, 35, (1911), P. 898-908.

[66] A. Einstein, “Die Grundlage der allgemeinen Relativitatstheorie”., Ann. Physik, Vol. 49, (1916), P. $769-822$.

[67] Robinson A. Non-standard analysis. -Princeton: Princeton Univ. Press, (1996), 293 p.

[68] Kanovei V. and Reeken M. Nonstandard analysis. Axiomatically. Berlin: SpringerVerlag, (2004), XVI+408 p.

[69] Gordon E. I., Kusraev A. G. Kutateladze S. S., Infinitesimal Analysis., Dordrecht etc.: Kluwer Academic Publishers, (2002), XIV+422 p.

[70] Yaglom I. M. "Complex Number and its Application in Geometry", Moscow: Fizmatgiz, (1963), 192 p., P. 13-19. // Complex Numbers in Geometry, Academic Press, (1968), 256 p.

[71] William K. Clifford, On the Space Theory of Matter, Proc. of the Cambridge Phil. Soc., 2, (1876), P. 157-158.

[72] D. Hestenes, G. Sobczyk, Clifford Algebra to Geometric Calculus - A Unified Language for Mathematical and Physics, Reidel Publishing Company, (1984), 336 p.

[73] Lavrentiev M. A., Shabat B. V., Problems of hydrodynamics and their mathematical models, Moscow: Science, (1973). 416 p., P. 50-56.

[74] Frobenius G., “Über das Pfaffische Probleme”, Jl. für die reine u. angew. Math. 82, (1877), P. 230-315.

[75] Max Tegmark: “The Mathematical Universe”, Found. Phys. 38, (2008), P. 101-150;

[76] V. S. Vladimirov, I. V. Volovich, Ye. I. Zelenov «p-Adic Analysis and Mathematical Physics». Singapore: World Scientific, (1994), 352 p.

[77] B. Dragivich, A. Yu. Khrennikov, S. V. Kosyrev, I. V. Volovich, E. I. Zelenov. p-Adic Mathematical Physics: The First 30 Years. p-Adic Numbers, Ultrametric Anal. Appl., 9:2 (2017), P. 87-121.

[78] J. Schwinger «A Magnetic Model of Matter», Science, Vol. 165 (N. 3895), (1969), P. 757-761.

[79] Mandelbrot B. B., The Fractal Geometry of Nature, San Francisco: W. H. Freeman and Co., (1982), 460 p.

[80] Robert L. Oldershaw. "Self-Similar Cosmological Model: Introduction and Empirical Tests" , International Journal of Theoretical Physics, Vol. 28, No. 6, (1989), P. 669-694. 
[81] Y. Baryshev, P. Teerikorpi. Discovery of Cosmic Fractals, World Scientific Publishing Co. Pte. Ltd., (2002), 363 p.

[82] G. M. Zaslavsky, R. Z. Sagdeev, D. A. Usikov, A. A. Chernikov, Weak Chaos and QuasiRegular Patterns., Cambridge: Cambridge University Press, (1991), 265 p.

[83] Kuznetsov S. P., Hyperbolic Chaos: A Physicist's View, Higher Education Press: Beijing and Springer-Verlag: Berlin, Heidelberg, (2012), 336 p.

[84] V. S. Vladimirov, I. V. Volovich, "Superanalysis. I. Differential Calculus", TMF, Vol. 59, N. 1, (1984), P. 3-27.

[85] I. V. Volovich, "p-adic Space-Time and String Theory", TMF, Vol. 71, N. 3, (1987), P. 337-340.

[86] Alexander G. Parkhomov. Space. Earth. Human.: New Views on Science., Moscow: Izdatel'stvo Nauka, (2009), 272 p., P. 20-38. // Independently published, (2019), 251 p.

[87] Gorban A. N., Kegl B., Wunsch D., Zinovyev A., Principal Manifolds for Data Visualization and Dimension Reduction., Vol. 58, Springer, Berlin-Heidelberg-New York, (2008), $340 \mathrm{p}$.

[88] Gorban A. N., Zinovyev A., Principal Graphs and Manifolds. In Handbook of Research on Machine Learning Applications and Trends: Algorithms, Methods and Techniques., (eds. Olivas E. S., Guererro J. D. M., Sober M. M., Benedito J. R. M., Lopes A. J. S.). Information Science References, Hershey, PA, (2009).

[89] Gorban A. N., Zinovyev A., Principal Manifolds and Graphs in Practice: from Molecular Biology to Dynamical Systems., Int. Journ. Neural Syst., 20, (2010), P. 219-232.

[90] Dirac P. A. M., Quantized singularities in the electromagnetic field, Proc. Roy. Soc. Ser. A, Vol. 133, Iss. 821, (1931), P. 60-72.

[91] Gerard 't Hooft, "Magnetic monopoles in unified gauge theories", Nuclear Physics B. 79 (2), (1974), P. 276-284.

[92] Polyakov A.M., "Particle spectrum in quantum field theory", Journal of Experimental and Theoretical Physics Letters, Vol. 20, (1974), P. 194.

[93] Coleman S., The Magnetic Monopole Fifty Years Later, UFN, Vol. 144, (1984), P. 277340 .

[94] P. A. R. Ade, N. Aghanim, M. I. R. Alves, C. Armitage-Caplan, M. Arnaud, M. Ashdown, F. Atrio-Barandela, J. Aumont, H. Aussel, C. Baccigalupi, et al. (Planck Collaboration). Planck 2013 results. I. Overview of products and scientific results, Astronomy \& Astrophysics, Vol. 571, A1, (2014), P. 48.

[95] N. Aghanim, M. Ashdown, J. Aumont, C. Baccigalupi, M. Ballardini, A. J. Banday, R. B. Barreiro, N. Bartolo, S. Basak, R. Battye, et al (Planck Collaboration), "Planck intermediate 
results. XLVI. Reduction of large-scale systematic effects in HFI polarization maps and estimation of the reionization optical depth", Astronomy \& Astrophysics, Vol. 596, A107, (2016), P. 52.

[96] Adam G. Riess, Lucas M. Macri, Samantha L. Hoffmann, Dan Scolnic, Stefano Casertano, Alexei V. Filippenko, Brad E. Tucker, Mark J. Reid, David O. Jones, Jeffrey M. Silverman, et al, "A 2.4\% Determination of the Local Value of the Hubble Constant", The Astrophysical Journal, Vol. 826, Issue 1, (2016), P. 33.

[97] Adam G. Riess, Stefano Casertano, Wenlong Yuan, Lucas M. Macri, and Dan Scolnic, "Large Magellanic Cloud Cepheid Standards Provide a 1\% Foundation for the Determination of the Hubble Constant and Stronger Evidence for Physics beyond $\Lambda$ CDM.", The Astrophysical Journal, Vol. 876, Issue 1, (2019), P. 85.

[98] Blokhintsev D. I., Space and time in the microworld, Moscow: Science, (1982), 347 p., P. 256-283.

[99] Carlo Rovelli, (2001). Quantum space-time: what we do know? (In C. Calender and N. Hugget (eds.), "Physics Meets Philosophy at the Planck scale", Cambridge University Press, P. 101-122.

[100] Terletsky, Ya. P., "Hypothesis of birth of particle pair with positive and negative masses from vacuum and its cosmological consequence", in the book "Problems of theoretical Physics", Moscow: publishing house UDN, (1990), P. 3-7.

[101] Abhay Ashtekar, "New Variables for Classical and Quantum Gravity”, Phys. Rev. Lett., 57(18), (1986), P. 2244-2247.

[102] Abhay Ashtekar, Jorge Pullin (eds.). Loop Quantum Gravity: The First 30 Years., (In 100 Years of General Relativity), World Scientific Publishing, (2017), 320 pp.

[103] Veneziano G., «Construction of a crossing-symmetric, Regge-behaved amplitude for linearly rising trajectories». Nuovo Cimento, 57 A, (1968), P. 190-197.

[104] I. V. Volovich, «p-Adic string», Classical Quantum Gravity, Vol. 4, (1987), P. 83-87.

[105] Kushner A., Lychagin V., Rubtsov V., Contact Geometry and Nonlinear Differential equations., Cambridge University Press, (2007), 496 p.

[106] G. M. Zaslavsky, Chaos in Dynamic Systems, New-York: Harwood Academic Publishers, (1985), 370 p.

[107] P. Candelas, Gary T. Horowitz, Andrew Strominger, Edward Witten. Vacuum configurations for superstrings., Nuclear Physics B, Vol. 258, (1985), P. 46-74.

[108] W. Heisenberg «Über die Spektra von Atomsystemen mit zwei Elektronen». Z. Phys. 39, (1926), P. 499-518. 
[109] P. W. Anderson, Theory of Magnetic Exchange Interactions, Exchange in Insulators and Semiconductors in Solid States Physics, Vol. 14, F. Seitz and D. Turnbull (eds.) Academic Press, New York, 1963.

[110] L. Landau, R. Pierts, Quantum Electrodynamics in Configuration Space, Zeit. f. Phys., Vol. 62, (1930), P. 188-200.

[111] A. T. Gavrilin, On the probability amplitude of photon's position, Vestnik of Lobachevsky State University of Nizhni Novgorod, N. 6(1), (2011), P. 70-74.

[112] S. L. McCall and E. L. Hahn, Self-induced Transparency by Pulsed Coherent Light, Phys. Rev. Lett., Vol. 18, (1967), P. 908-911.

[113] C. N. Yang, R. Mills. Conservation of Isotopic Spin and Isotopic Gauge Invariance, Phys. Rev., Vol. 96, N. 1, (1954), P. 191 - 195.

[114] Schwinger J. A Theory of the Fundamental Interactions, Ann. of Phys., Vol. 2, (1957), P. 407-434.

[115] J. Leite - Lopes, A model of the universal fermi interaction, Nucl. Phys., Vol. 8, (1958), P. 234-236.

[116] Salam A., Ward J. C., Weak and Electromagnetic Interactions, Nuovo Cimento, Vol. 11, (1959), P. 568-577.

[117] Sheldon L. Glashow, Partial-symmetries of weak interactions, Nucl. Phys., Vol. 22, (1961), P. 579-588.

[118] Salam A., Ward J. C., Electromagnetic and weak interactions, Phys. Lett., Vol. 13, (1964), P. 168-171.

[119] Faddeev L. D., Popov V. N. Feynman Diagrams for the Yang-Mills Field, Phys. Lett. B, Vol. 25, (1967), P. 29 - 30.

[120] Weinberg S., A model of leptons, Phys. Rev. Lett., Vol. 19, (1967), P. 1264-1266.

[121] Salam A., Week and Electromagnetic Interaction, Elementary Particle Theory, Ed. N. Svartholm. - Almguist and Wiksell, Stockh., (1968), P. 367-377.

[122] G. 't Hooft, Renormalization of massless Yang-Mills fields, Nucl. Phys., Vol. B33, (1971), P. 173-199.

[123] G. 't Hooft, Renormalizable Lagrangians for massive Yang-Mills fields, Nucl. Phys., V. B35, (1971), P. 167-188.

[124] G. 't Hooft, M. Veltman. Regularization and Renormalization of Gauge Fields, Nuclear Physics B, Vol. 44, (1972), P. 189-219.

[125] L. D. Faddeev, Gauge Invariant Model of Electromagnetic and Week Interactions of Leptons, Report of Ac. of Sc. USSR, Vol. 210, N. 4, (1973), P. 807- 810. 
[126] G. Arnison, O.C. Allkofer, A. Astbury, Bernard Aubert, C. Bacci, G. Bauer, A. Bezaguet, R.K. Bock, T.J.V. Bowcock, M. Calvetti, et al. (UA1), Experimental observation of isolated large transverse energy electrons with associated missing energy at s=540 GeV, Phys. Lett. B, Vol. 122, (1983), P. 103-116.

[127] M. Banner, R. Battiston, P. Bloch, F. Bonaudi, K. Borer, M. Borghini, J.C. Chollet, A.G. Clark, C. Conta, P. Darriulat, et al. (UA2), Observation of single isolated electrons of high transverse momentum in events with missing transverse energy at the CERN pp collider, Phys. Lett. B, Vol. 122, (1983), P. 476-485.

[128] G. Arnison, A. Astbury, Bernard Aubert, C. Bacci, G. Bauer, A. Bezaguet, R.K. Bock, T.J.V. Bowcock, M. Calvetti, et al. (UA1), Experimental observation of lepton pairs of invariant mass around $95 \mathrm{GeV} / \mathrm{c} 2$ at the CERN SPS collider, Phys. Lett. B, Vol. 126, (1983), P. 398-410.

[129] P. Bagnaia, M. Banner, R. Battiston, Ph. Bloch, F. Bonaudi, K. Borer, M. Borghini, J.-C. Chollet, A. G. Clark, C. Conta, et al. (UA2), Evidence for Z0 ---> e+ e- at the CERN anti-p p Collider, Phys. Lett. B, Vol. 129, (1983), P. 130-140.

[130] G. Arnison, A. Astbury, Bernard Aubert, C. Bacci, G. Bauer, A. Bezaguet, R. Bock, T.J.V. Bowcock, M. Calvetti, P. Catz, et al. (UA1), Further evidence for charged intermediate vector bosons at the SPS collider, Phys. Lett. B, Vol. 129, (1983), P. 273-282.

[131] P. Bagnaia, M. Banner, R. Battiston, Ph. Bloch, F. Bonaudi, K. Borer, M. Borghini, J. Burger, P. Cenci, J.-C. Chollet, et al. (UA2), A Study of High Transverse Momentum Electrons Produced in anti-p p Collisions at 540-GeV, Z. Phys. C (1), Vol. 24, (1984), P. 1-17.

[132] G. B. Andresen, M. D. Ashkezari, M. Baquero-Ruiz, W. Bertsche, P. D. Bowe, E. Butler, C. L. Cesar, S. Chapman, M. Charlton, A. Deller, S. Eriksson, et al. Trapped antihydrogen. Nature 468, (2010), P. 673-676.

[133] G.B. Andresen, M.D. Ashkezari, M. Baquero-Ruiz, W. Bertsche, E. Butler, C.L. Cesar, A. Deller, S. Eriksson, J. Fajans, T. Friesen, et al. Confinement of antihydrogen for 1,000 seconds. Nat. Phys. 7, (2011), P. 558-564.

[134] The ALPHA Collaboration \& Charman A. E. Description and first application of a new technique to measure the gravitational mass of antihydrogen. Nat. Commun., Vol. 4, (2013), 1785.

[135] P. Pérez, D. Banerjee, F. Biraben, D. Brook-Roberge, M. Charlton, P. Cladé, P. Comini, P. Crivelli, O. Dalkarov, P. Debu, et al. The GBAR antimatter gravity experiment. Hyperf. Interact., Vol. 233, (2015), P. 21 - 27.

[136] Bertsche, W. A. Prospects for comparisons of matter and antimatter gravitation with ALPHA-g., Philosophical Transactions of The Royal Society A., (2018), 376 (2116): 20170265. 
[137] Kostelecký V. A., Vargas A. J. Lorentz and CPT tests with hydrogen, antihydrogen, and related systems. Phys. Rev. D, Vol. 92, 056002, (2015).

[138] S. Ulmer, C. Smorra, A. Mooser, K. Franke, H. Nagahama, G. Schneider, T. Higuchi, S. Van Gorp, K. Blaum, Y. Matsuda, W. Quint, J. Walz \& Y. Yamazaki. High-precision comparison of the antiproton-to-proton charge-to-mass ratio. Nature, Vol. 524, (2015), P. $196-199$.

[139] Crivelli P., Cooke D., Heiss M. W. Antiproton charge radius. Phys. Rev. D, Vol. 94, (2016), 052008.

[140] M. Hori, H. Aghai-Khozani, A. Soter, D. Barna, A. Dax, R. Hayano, T. Kobayashi, Y. Murakami, K. Todoroki, H. Yamada, D. Horvath, L. Venturelli. Buffer-gas cooling of antiprotonic helium to 1.5 to $1.7 \mathrm{~K}$, and antiproton-to-electron mass ratio. Science, Vol. 354, (2016), P. $610-614$.

[141] M. Ahmadi, B. X. R. Alves, C. J. Baker, W. Bertsche, E. Butler, A. Capra, C. Carruth, C. L. Cesar, M. Charlton, S. Cohen, et al. Observation of the 1S-2S transition in trapped antihydrogen. Nature, Vol. 541, (2017), P. 506 - 510.

[142] M. Ahmadi, B. X. R. Alves, C. J. Baker, W. Bertsche, E. Butler, A. Capra, C. Carruth, C. L. Cesar, M. Charlton, S. Cohen, et al. Observation of the hyperfine spectrum of antihydrogen. Nature, Vol. 548, (2017), P. 66 - 69.

[143] M. Ahmadi, B. X. R. Alves, C. J. Baker, W. Bertsche, E. Butler, A. Capra, C. Carruth, C. L. Cesar, M. Charlton, S. Cohen, et al. Antihydrogen accumulation for fundamental symmetry tests. Nat. Commun., Vol. 8, (2017), P. 681.

[144] Smorra, C. A parts-per-billion measurement of the antiproton magnetic moment. Nature, Vol. 550, (2017), P. 371 - 374.

[145] Rasmussen C. 0., Madsen N. \& Robicheaux F. Aspects of 1S-2S spectroscopy of trapped antihydrogen atoms. J. Phys. B, Vol. 50, 184002, (2017); corrigendum Vol. 51, 099501, (2018).

[146] M. Ahmadi, B. X. R. Alves, C. J. Baker, W. Bertsche, A. Capra, C. Carruth, C. L. Cesar, M. Charlton, S. Cohen, R. Collister, et al. Characterisation of the 1S-2S transition in antihydrogen. Nature, Vol. 557, (2018), P. 71 - 75.

[147] M. Ahmadi, B. X. R. Alves, C. J. Baker, W. Bertsche, A. Capra, C. Carruth, C. L. Cesar, M. Charlton, S. Cohen, R. Collister, et al. Observation of the 1S-2P Lyman- $\alpha$ transition in antihydrogen. Nature, Vol. 561, (2018), P. 211-215.

[148] Erikson S. Precision measurements on trapped antihydrogen in the ALPHA experiment. Philos. Trans. Royal Soc. A, Vol. 376, (2018), 20170268.

[149] Capra A. \& ALPHA Collaboration. Lifetime of magnetically trapped antihydrogen in ALPHA. Hyperfine Interact. 240, 9 (2019). 
[150] S. Sala, A. Ariga, A. Ereditato, R. Ferragut, M. Giammarchi, M. Leone, C. Pistillo, P. Scampoli «First demonstration of antimatter wave interferometry», Science Advances, Vol. 5, No. 5, eaav7610, (2019).

[151] The ALPHA Collaboration. Investigation of the fine structure of antihydrogen. Nature 578, (2020), P. 375-380.

[152] R. P. Feynman and M. R. Hibbs, Quantum mechanics and path integrals, (McGraw Hill, New York, 1965), 365 p.

[153] Handjian Ling, Guillam E. Mclvor, Kasper van der Vaart, Richard T. Vaughan, Alex Thornton, Nicholas T. Ouellette, Costs and Benefits of Social Relationships in the Collective Motion of Bird Flocks, Nature Ecology \& Evolution, 3, (2019), P. 943-948.

[154] Nicolas Manton and Paul Sutcliffe, Topological Solitons. Cambridge University Press, N.-Y., (2004), 506 p.

[155] V. E. Zakharov, A. B. Shabat, Exact Theory of Two-dimensional Self-focusing and Onedimensional Self-modulation of Waves in Nonlinear Media, JETP Letter, Vol. 61, N. 1, (1971), P. 118-134.

[156] V. E. Zakharov, L. A. Takhtadzhyan, L.D. Faddeev, Complete description of solutions of the 'sine-Gordon' equation, Sov. Phys. Dokl., Vol. 219, N. 6, (1974), P. 1334-1337.

[157] Zakharov V. E. (ed.) “What is integrability?”, Springer-Verlag, (1992), XIV+321 p.

[158] V. E. Zakharov, E. A. Kuznetsov, Solitons and collapses: two evolution scenarios of nonlinear wave systems, UFN, Vol. 182, (2012), P. 569 - 592.

[159] O. M. Braun, Y. S. Kivshar, The Frenkel-Kontorova Model. Concept, Methods and Applications. SPRINGER, (2004), 536 p.

[160] A. S. Fokas «Integrable Nonlinear Evolution Partial Differential Equation in $4+2$ and 3+1 Dimensions», Phys. Rev. Lett., Vol. 96, (2006), 190201. 


\section{Acknowledgment}

I dedicate this work to my father Shamail Sh. Avshalumov (1937-2012), PhD (1983, technical science) and my mother Malka Yus. Avshalumova (1941), chemist. 